\title{
USING A HIGH REPETITION RATE Nd:YAG LASER FOR PULSED LASER ABLATION PROPULSION
}

\author{
by \\ Daniel Hayek \\ B.Eng, Ryerson University, 2008 \\ A thesis \\ presented to Ryerson University \\ in partial fulfillment of the \\ requirements for the degree of \\ Master of Applied Science
}

in the Program of

Aerospace Engineering

Ryerson University

Toronto, Ontario, Canada

(c) Copyright by Daniel Hayek, 2010 


\title{
Author's Declaration
}

I hereby declare that I am the sole author of this thesis report.

I authorize Ryerson University to lend this thesis to other institutions or individuals for the purpose of scholarly research.

\section{Daniel Hayek}

Department of Aerospace Engineering

Ryerson University

I further authorize Ryerson University to reproduce this project by photocopying or by other means, in total or in part, at the request of other institutions or individuals for the purpose of scholarly research.

\author{
Daniel Hayek \\ Department of Aerospace Engineering \\ Ryerson University
}




\section{Using a High Repetition Rate Nd:YAG Laser for Pulsed Laser Ablation Propulsion \\ Master of Applied Science \\ In the program of \\ Aerospace Engineering \\ 2010}

Daniel Hayek

School of Graduate Studies

Ryerson University

\section{Abstract}

Laser propulsion is a relatively new technology being researched for its vast potential. A test apparatus was developed to measure small forces using piezoelectric films and a developed amplifier tuned to the specific frequencies expected from the system. The system provided consistent results comparable to published values.

The tests conducted in this thesis evaluated the effects of using a high repetition rate laser for laser propulsion. The results are on the micropropulsion scale, however, the findings are expected to perform similarly on a larger scale. The thrust, moment coupling coefficient, and specific impulse values were evaluated for aluminum, brass, and PVC of differing thicknesses. The results concluded that the repetition rate in fact did not have much effect on the thrust; thrust was primarily dependent on the pulse energy and the material thickness. The repetition rate was found to affect the specific impulse values; a result of the heat affected zone created by the laser ablation, thereby reducing the effective propellant used for propulsion. 


\section{Acknowledgements}

I would like to thank my supervisor Dr. Bo Tan for all of her guidance and support in this thesis. She allowed me to direct the research for my personal interests and continually supported my thoughts and ideas. Her constructive criticism helped me overcome some heavy obstacles in the development of this thesis.

I would like to thank my co-supervisor Dr. Jeffrey Yokota for his guidance throughout the thesis as he helped direct my thoughts when I was debating concepts. I would also like to thank Dr. Krishnan Venkatakrishnan for his guidance with the laser set up, and operation, as well as theoretical and experimental guidance for laser concepts.

I would like to thank Mr. Primoz Cresnik for his extensive assistance with this thesis. Without his experimental experience and electronics knowledge the new designed method of testing would not have been possible. His assistance throughout this thesis was invaluable.

Finally, to my friends and family who stood by me throughout this thesis. Their support and words of encouragement urged me to dive deeper into the topic, and helped complete this thesis. 


\section{Table of Contents}

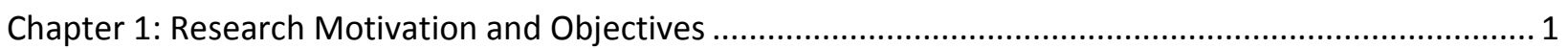

$1.1 \quad$ Introduction to Laser Micropropulsion ................................................................................. 1

1.2 Purpose for Laser Micropropulsion Research ........................................................................ 2

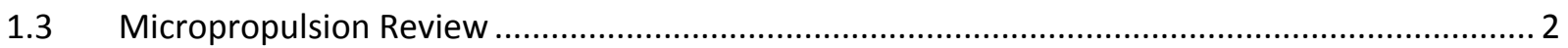

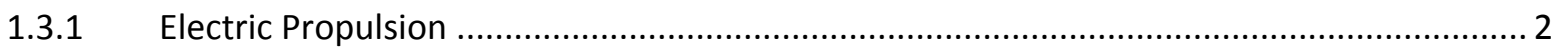

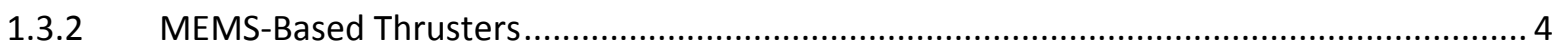

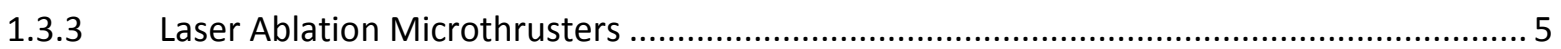

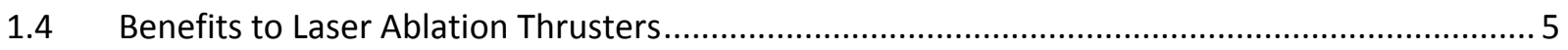

1.4.1 Important Laser Parameters for Evaluation............................................................... 6

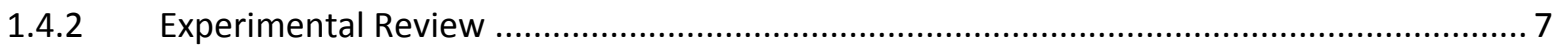

1.5 New Trends in Micropropulsion Research .......................................................................... 12

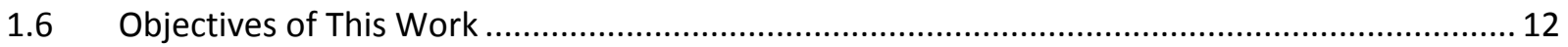

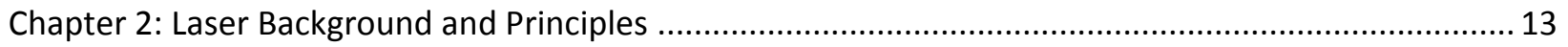

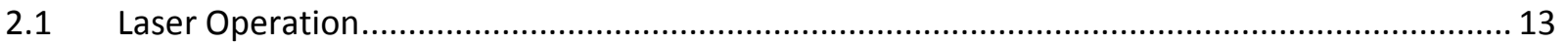

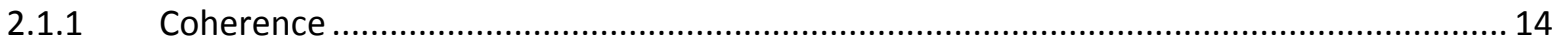

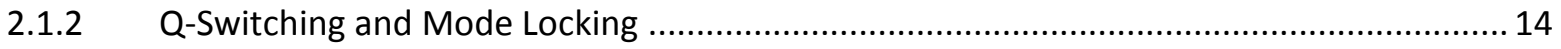

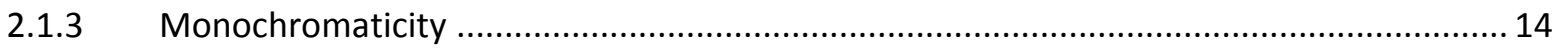

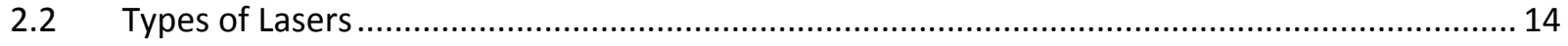

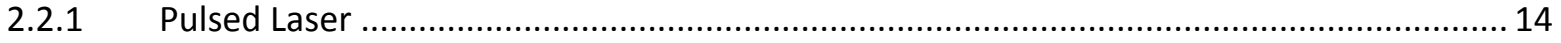

2.2.2 Femtosecond and Nanosecond Pulsed Lasers ........................................................... 15

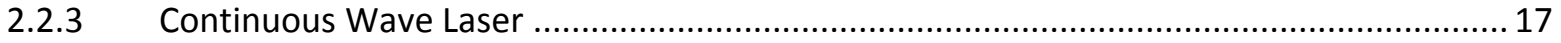

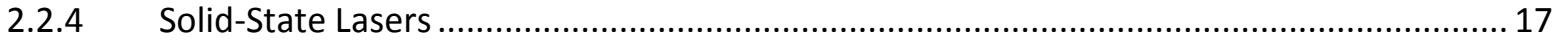

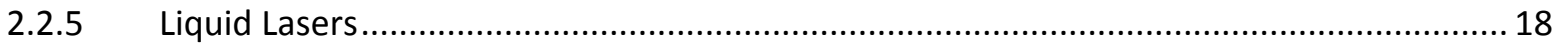




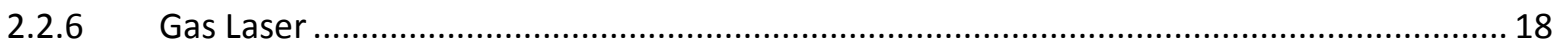

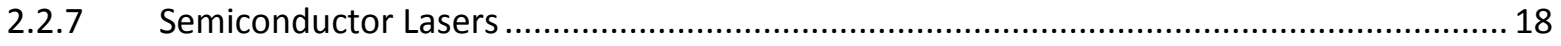

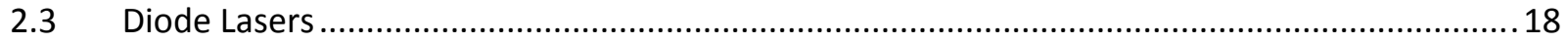

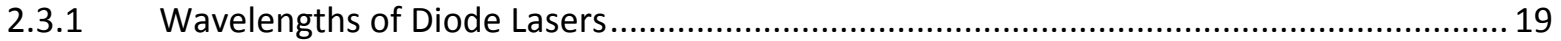

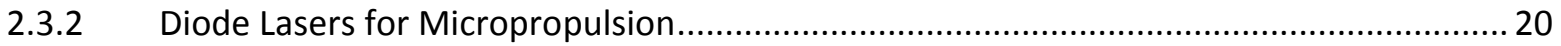

Chapter 3: Fundamentals of Laser Ablation Propulsion ....................................................................... 22

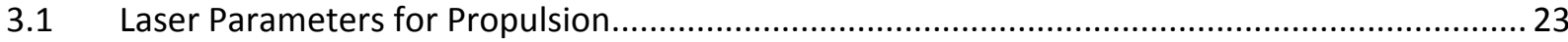

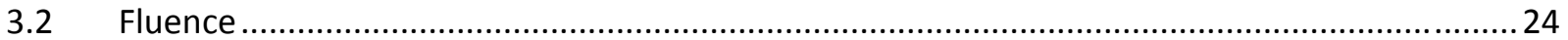

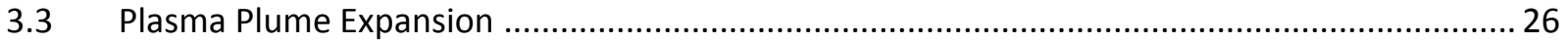

3.4 Modes of Laser Ablation Plume Generation ...................................................................... 27

3.5 Propellants Used for Laser Driven Micropropulsion ........................................................... 29

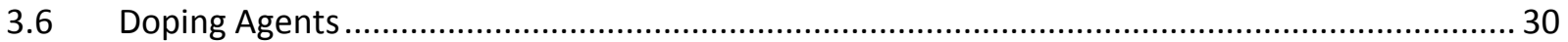

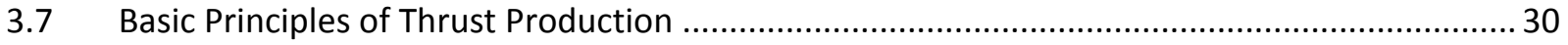

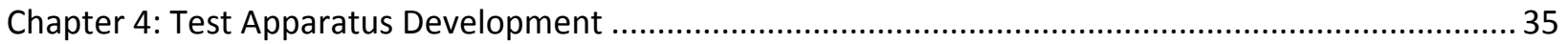

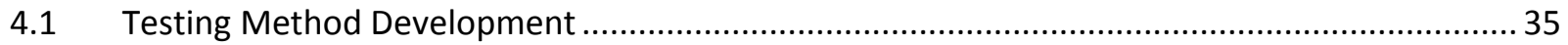

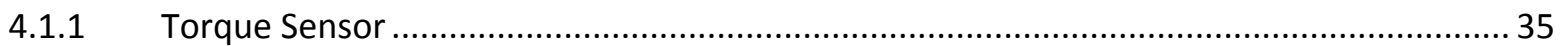

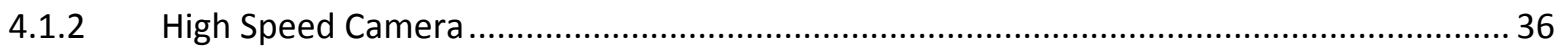

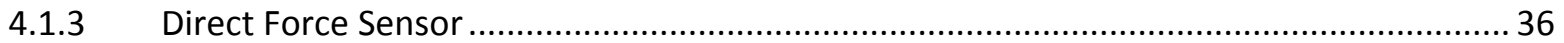

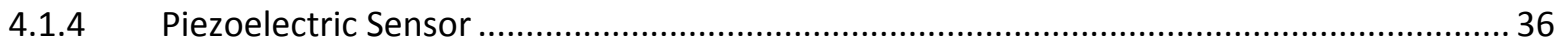

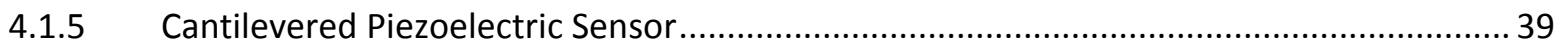

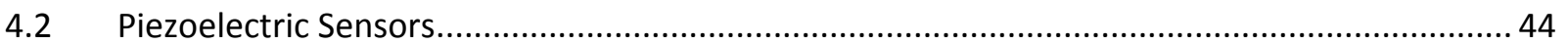

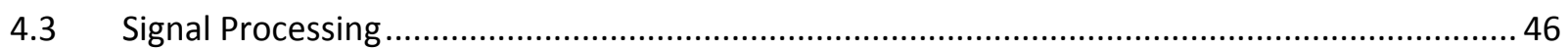

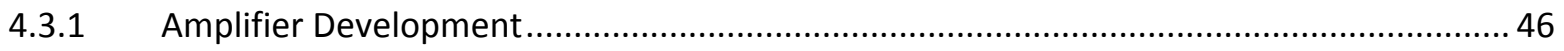

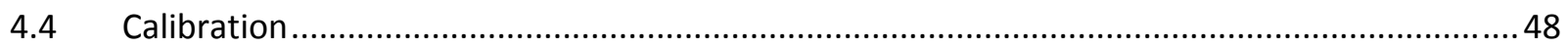

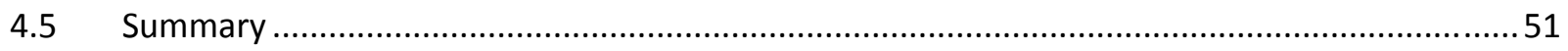

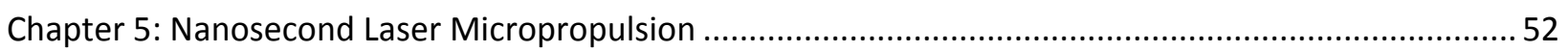




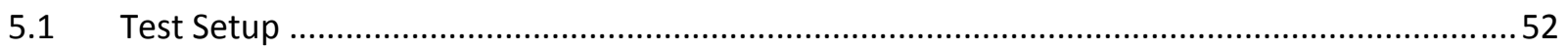

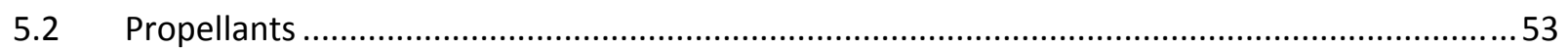

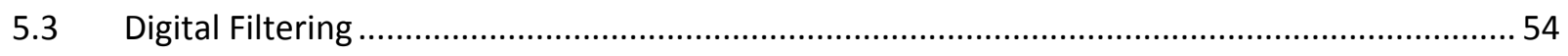

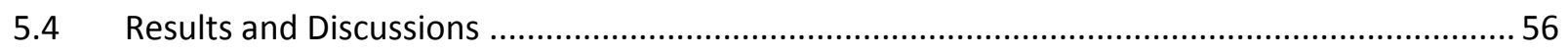

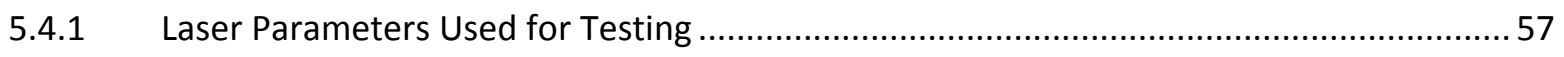

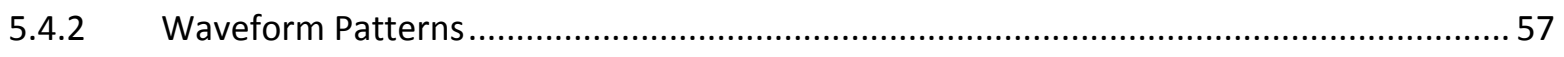

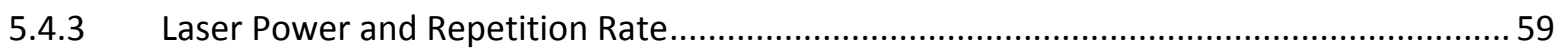

5.4.4 Moment Coupling Coefficient and Specific Impulse .................................................. 64

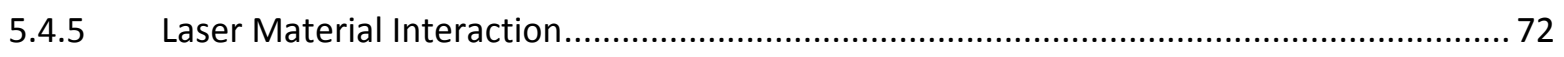

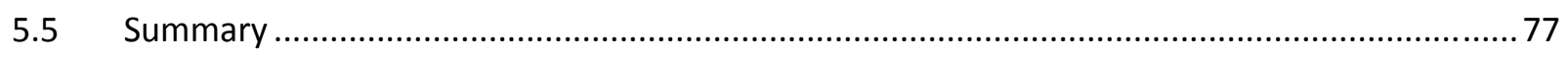

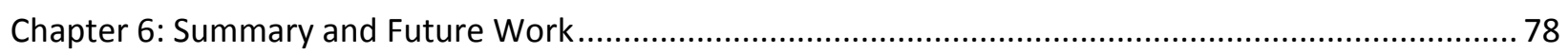

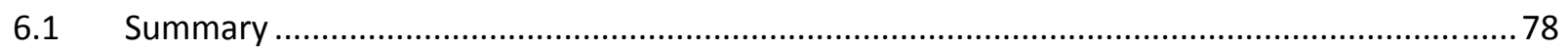

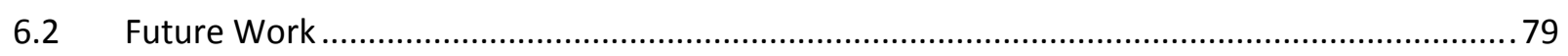

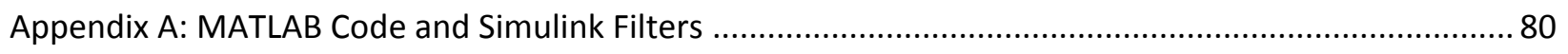

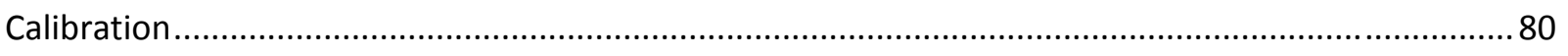

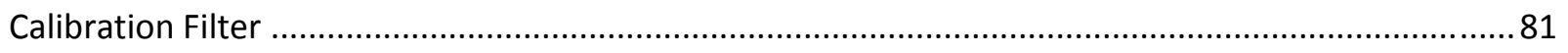

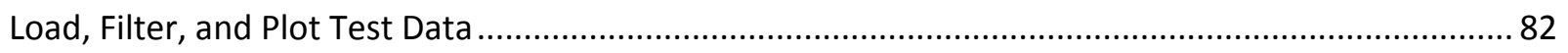

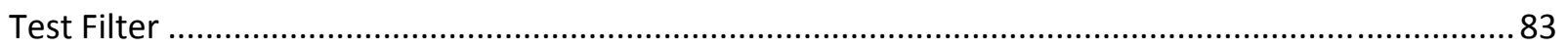

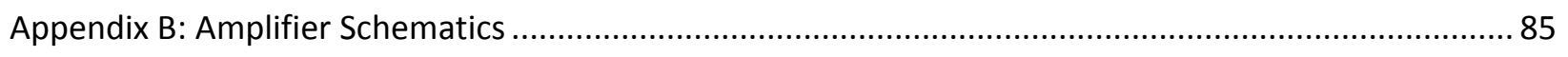

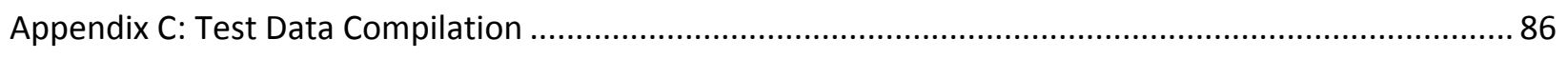

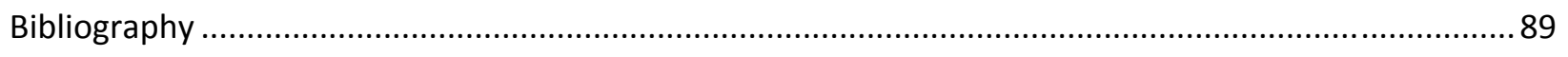




\section{List of Figures}

Figure 1-1: MEMS-based Microthruster from Zhang et al................................................................ 4

Figure 1-2: Independent Laser Powering Satellite [12] .................................................................... 6

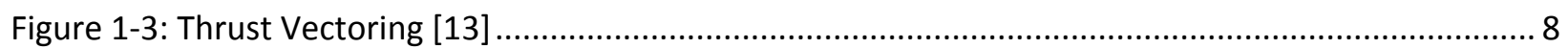

Figure 2-1: Chirped Pulse Amplification Operation Diagram [31] .......................................................... 15

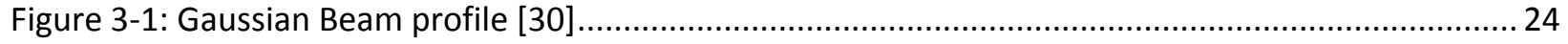

Figure 3-2: Intensity of Gaussian Beam Across Beam Diameter [30] .................................................... 25

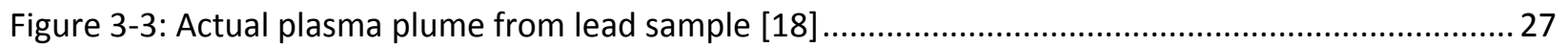

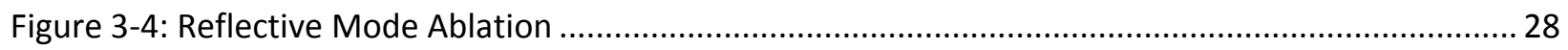

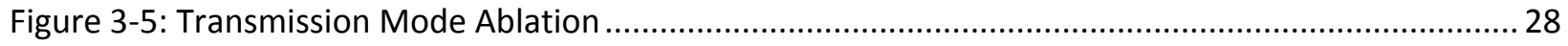

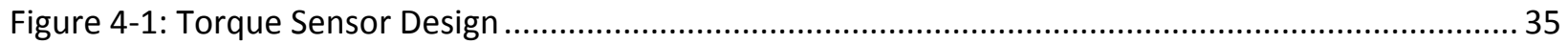

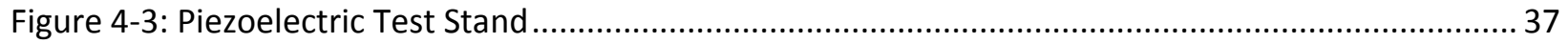

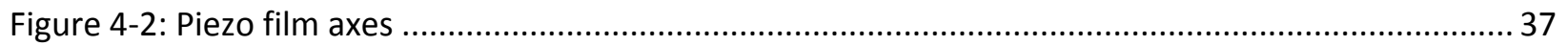

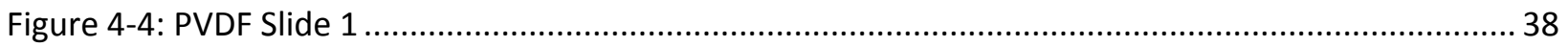

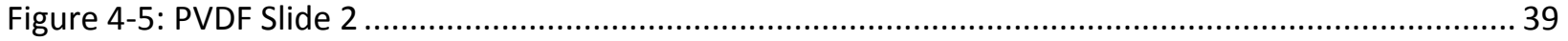

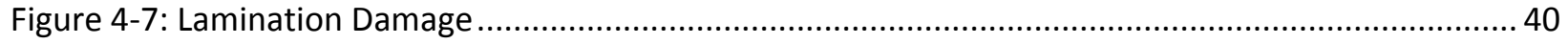

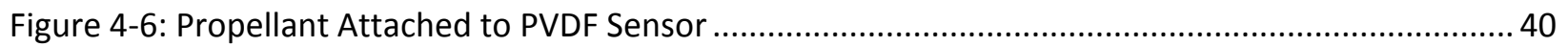

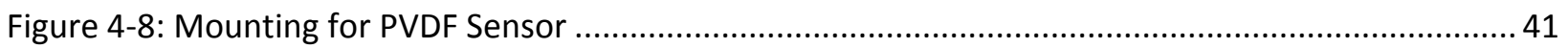

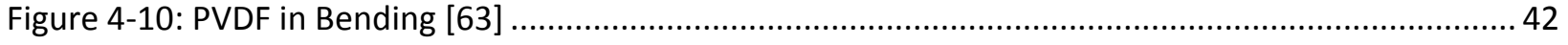

Figure 4-9: Applying a Force Along the 3 Axis..................................................................................... 42

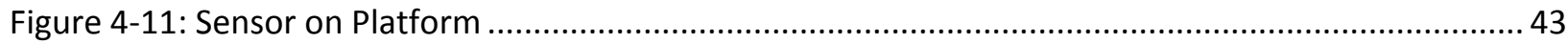

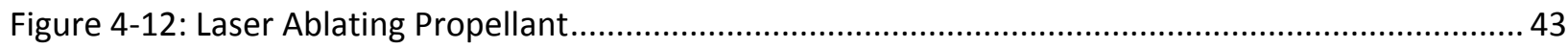

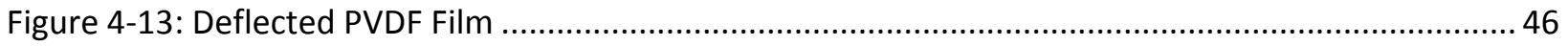

Figure 4-14: Vargas et al. Circuit on a Breadboard ............................................................................. 47

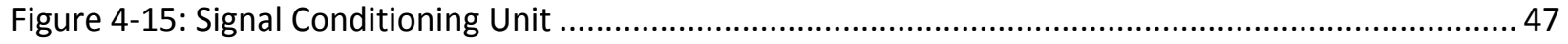

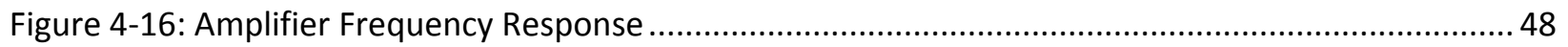

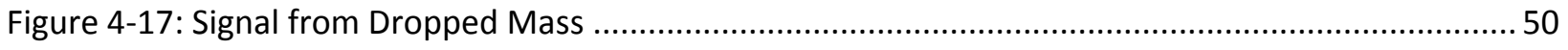

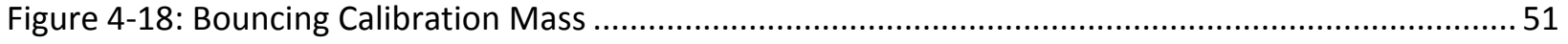

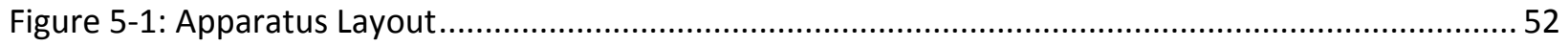

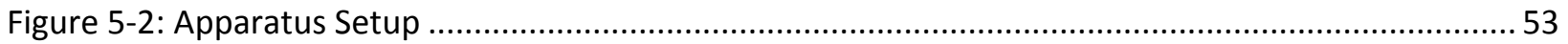


Figure 5-3: Unfiltered and Filtered Signals for Brass, $0.001 ", 121 \mu \mathrm{J}$ at $10 \mathrm{kHz}$

Figure 5-4: Unfiltered on Filtered Signals for Brass, $0.001 ", 121 \mu \mathrm{J}$ at $10 \mathrm{kHz}$ using bandpass filter .......... 56

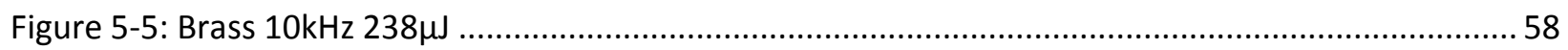

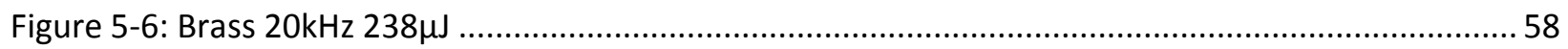

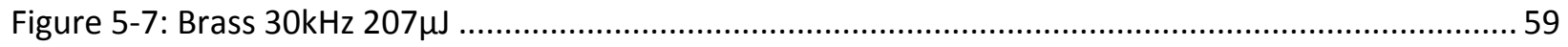

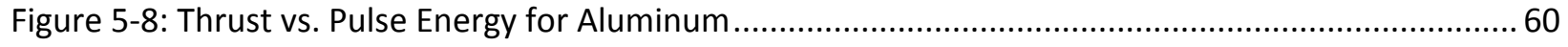

Figure 5-9: Thrust vs. Pulse Energy for PVC Logarithmic ........................................................... 61

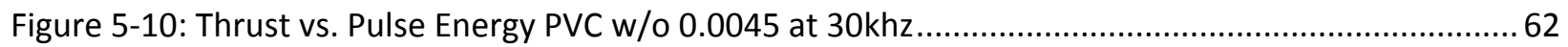

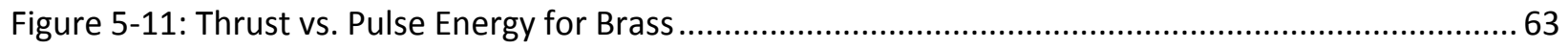

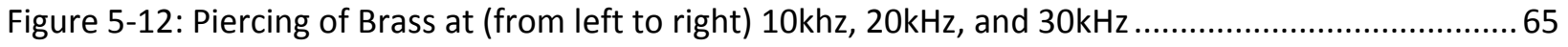

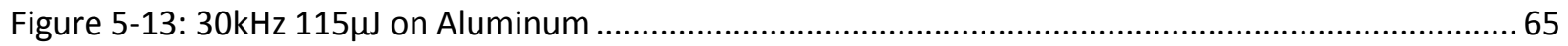

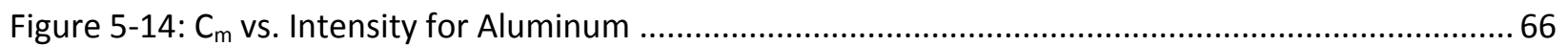

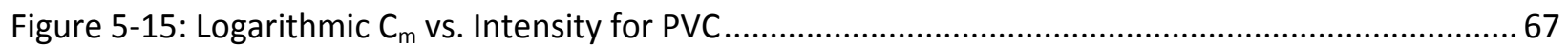

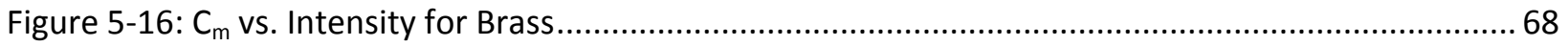

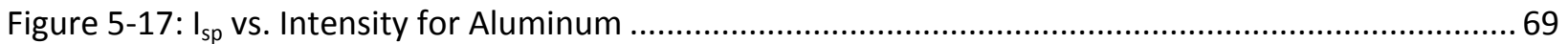

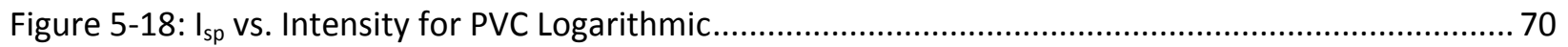

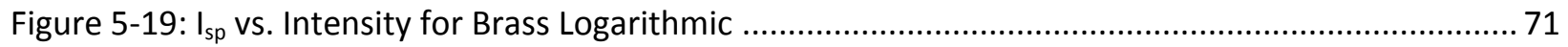

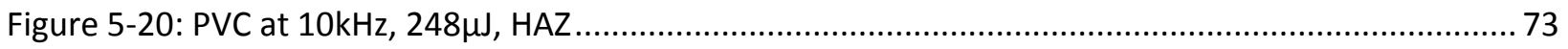

Figure 5-21: PVC (Top Left), 20kHz (Top Right), 30kHz (Bottom Left), and 40kHz (Bottom Right)........... 74

Figure 5-22: Brass at $10 \mathrm{kHz}$ (Top Left), 20kHz (Top Right), 30kHz (Bottom Left), and 40kHz (Bottom Right) 75

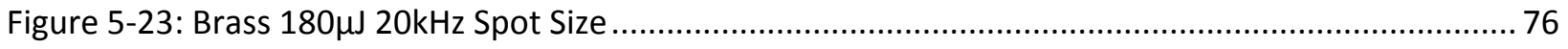

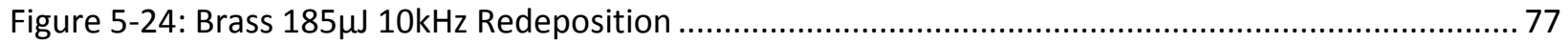




\section{List of Tables}

Table 1-1: Luke \& Phipps 2003 Test Parameters ................................................................................... 7

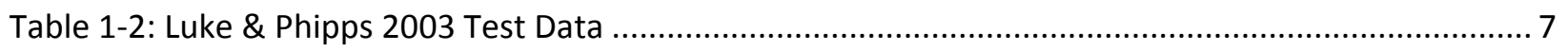

Table 1-3: Phipps, Luke, Helgeson, Jonhson, 2006 Test Data .................................................................... 8

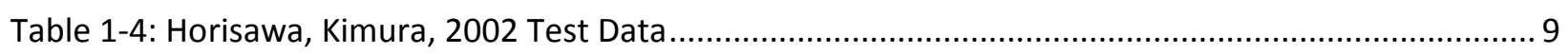

Table 1-5: Pakhomov, Gregory, Thompson 2002 Test Parameters ........................................................ 9

Table 1-6: Maesato, Koizumi, \& Tahara, 2006 Test Data............................................................................ 11

Table 1-7: Yokoyama, Horisawa, Funaki, \& Kuninaka, 2007 Test Data................................................... 11

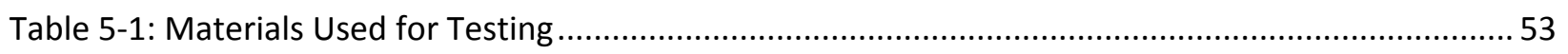

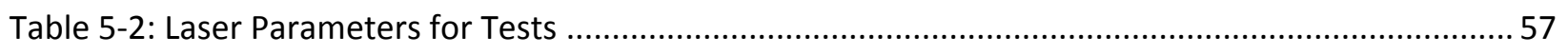

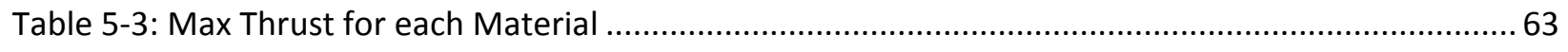

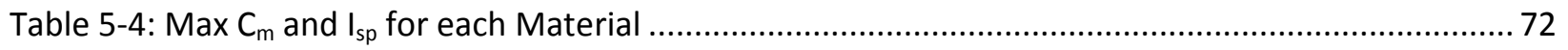




\section{Nomenclature}

\begin{tabular}{|c|c|c|}
\hline$A$ & absorptivity & \\
\hline$A_{e}$ & nozzle exit area & \\
\hline$\alpha$ & material absorption coefficient & \\
\hline DOF & depth of focus & \\
\hline $\mathrm{cm}$ & centimeter & $10^{-2} \mathrm{~m}$ \\
\hline$C_{m}$ & moment coupling coefficient & \\
\hline $\mathrm{D}$ & collimated beam diameter & \\
\hline $\mathrm{dt}$ & change in time & \\
\hline$E$ & pulse energy & \\
\hline$f$ & focal length of lens & \\
\hline $\mathrm{F}$ & force & \\
\hline $\mathrm{Fl}(\mathrm{r})$ & fluence & \\
\hline $\begin{array}{l}\mathrm{Fl}_{\text {th }} \\
\text { fs }\end{array}$ & $\begin{array}{l}\text { material fluence threshold } \\
\text { femtosecond }\end{array}$ & $10^{-15} \mathrm{~s}$ \\
\hline go & gravitational acceleration & \\
\hline GW & gigawatt & $10^{9} \mathrm{~W}$ \\
\hline $\mathrm{h}$ & height for calibration mass & \\
\hline HAZ & heat affected zone & \\
\hline $\mathrm{Hz}$ & hertz & \\
\hline I & impulse & \\
\hline$I_{s p}$ & specific impulse & \\
\hline J & joule & \\
\hline $\mathrm{kHz}$ & kilohertz & $10^{3} \mathrm{~Hz}$ \\
\hline kJ & kilojoule & $10^{3} \mathrm{~J}$ \\
\hline kW & kilowatt & $10^{3} \mathrm{~W}$ \\
\hline$\lambda$ & wavelength of light & \\
\hline LSC & laser supported combustion & \\
\hline LSD & laser supported detonation & \\
\hline $\mathrm{m}$ & mass & \\
\hline$\dot{m}$ & mass flow rate & \\
\hline $\mathrm{MHz}$ & megahertz & $10^{6} \mathrm{~Hz}$ \\
\hline MJ & megajoule & $10^{6} \mathrm{~J}$ \\
\hline $\mathrm{mm}$ & millimeter & $10^{-3} \mathrm{~m}$ \\
\hline $\mathrm{mN}$ & millinewton & $10^{-3} \mathrm{~N}$ \\
\hline MW & megawatt & $10^{6} \mathrm{~W}$ \\
\hline $\mathrm{N}$ & Newton & \\
\hline Ns & Newton-second & \\
\hline$\eta_{\mathrm{ab}}$ & ablation efficiency & \\
\hline $\mathrm{nN}$ & nanonewton & $10^{-9} \mathrm{~N}$ \\
\hline ns & nanosecond & $10^{-9} \mathrm{~s}$ \\
\hline$P$ & momentum & \\
\hline$p_{e}$ & exit pressure & \\
\hline$p_{o}$ & atmospheric pressure & \\
\hline ps & picosecond & $10^{-12} \mathrm{~s}$ \\
\hline
\end{tabular}




$\begin{array}{lll}\text { PVC } & \text { polyvinyl chloride } & \\ \text { PVDF } & \text { polyvinylidene fluoride } & \\ Q^{*} & \text { energy per unit mass for ablation } & \\ R & \text { surface reflectivity } & \\ S & \text { laser heating source term } & \\ T & \text { thrust } & \\ t & \text { time } & 10^{12} \mathrm{~W} \\ T W & \text { terawatt } & 10^{-6} \mathrm{~m} \\ \mu m & \text { micrometer } & 10^{-6} \mathrm{~N} \\ \mu N & \text { micronewton } & \\ V & \text { repetition rate } & \\ V_{\text {ex }} & \text { effective exhaust velocity of ablated } \\ & \text { propellant } & \\ V_{\text {impact }} & \text { impact velocity of calibration mass } \\ V_{i} & \text { initial velocity of calibration mass } \\ V_{f} & \text { final velocity of calibration mass } \\ W & \text { watt } & \\ W_{0} & \text { spot size }\end{array}$




\section{Chapter 1: Research Motivation and Objectives}

\subsection{Introduction to Laser Micropropulsion}

Laser propulsion was first proposed by Dr. Arthur Kantrowitz in 1972 [1] with the idea of using a ground based high power laser to propel a flying object. The Myrabo Laser Lightcraft, designed by Larson et al, uses Kantrowitz's theory and has achieved lift off using a high power $\mathrm{CO}_{2}$ laser [2].

Laser Ablation Propulsion is a relatively new form of electric micropropulsion that has great potential due to the high efficiency of energy conversion. Micro propulsion is being developed for satellite maneuverability. Smaller satellites can be maneuvered with very small thrust pulses while in orbit, requiring a small amount of propellant for each maneuver. Other propulsion methods, such as chemical, solid and hybrid rockets, cannot be efficiently applied to a small satellite system as they require much more propellant than electric propulsion methods.

Lasers can produce large amounts of power in very short pulses making them useful for thruster applications where specific amounts of thrust are required [3]. This is best used for maneuvering due to the relatively low amount of power available at this point in time when compared to a chemical rocket. Laser propulsion dominates with respect to specific impulse, $I_{s p}$, and moment coupling coefficient, $C_{m}$, over chemical rocket propulsion. Specific impulse values for chemical rockets are typically limited to around 500s because of available reaction temperatures; lasers do not have a temperature limit, and thus $I_{s p}$ values greater than 5000 s are attainable [4].

Laser light can be focused to a very small point greatly increasing the density of the light beam [3]; this implies that more energy deposited in the material will increase the exhaust velocity of the particles and could provide more thrust. For the same amount of thrust, less propellant could be used due to focusing the laser energy [5]. Obviously, a higher power laser could produce very high energy densities when focused, but this also means that very small lasers can produce sufficient amounts of energy required for ablation. Benefits to using a smaller, low powered laser are that total mass requirements may be low and thus a lightweight diode laser could be used.

Satellite lifetime is currently propellant limited. If the amount of propellant on-board can be increased then the satellite lifetime can in turn be increased, thus decreasing costs for satellite replacement [6]. 
Many polymers have been tested for thrust generation and have been found to produce a generous amount of thrust when compared to metals. For example, to reach the ignition threshold of polymer materials used for micro propulsion requires just over $1 \mathrm{~W}$ of laser energy. For optimum moment coefficient values, a laser above $5 \mathrm{~W}$ could be used [7].

\subsection{Purpose for Laser Micropropulsion Research}

Space exploration and the satellite industry is one of extreme expense. For example, the costs of sending a satellite into geosynchronous orbit were upwards of $\$ 30,000$ per kilogram in 2001 [6]. Thrust values are still fairly low to actually transport the system to orbit from earth with an onboard laser, but are able to supply sufficient impulse for maneuvering. Electric propulsion and laser propulsion are sought after for their high specific impulse potential, which is regarded as the efficiency of an engine or rocket. It is effectively the change in impulse per unit weight of propellant. Micropropulsion is a viable means for satellite maneuvering due to its low system weight and energy requirements.

\subsection{Micropropulsion Review}

In 1958, Explorer I, and Vanguard I were the first microsatellites weighing in at $15 \mathrm{~kg}$ and $1.5 \mathrm{~kg}$ respectively [8]. As communications technology advanced, satellite pointing and station keeping became crucial. The requirement for systems to be able to maneuver a microsatellite efficiently was essential, and is still researched extensively to find more efficient methods. Generally, a satellite's lifetime is limited to the amount of propellant onboard. If the total impulse is increased to keep the satellite in orbit for a longer period of time, it would reduce hefty launch costs. The total impulse is the maximum amount of impulse that the system can provide over its operational lifetime. The other important parameter is the impulse bit, which is effectively the smallest amount of thrust that can be generated over the shortest period of time the propulsion system can fire [8]. This is important for precision maneuvering, and pulsed systems are best fit for these applications [6]. Micropropulsion is achieved by many different methods. This section outlines several types of micropropulsion systems and explains basic operation, as well as some benefits and performance data.

\subsubsection{Electric Propulsion}

Electric propulsion encompasses any propulsion technology that uses electricity to increase exhaust velocity [9]. Electric propulsion aims to increase the exhaust velocity to reduce the amount of propellant required to achieve the required mission thrust [9]. 
Several different technologies have been developed to produce low milli-Newton thrust levels. The three types of electric propulsion are electrothermal, electrostatic, and electromagnetic.

\subsubsection{Electrothermal Thrusters}

Electrothermal propulsion is achieved by electrically heating the propellant and allowing the exhaust to thermally expand, typically through a nozzle. The resistojet and the arcjet are two types of electrothermal propulsion. The resistojet heats the propellant via convection by passing electricity through a high resistance component and dissipating energy. Resistojet performance is around $300 \mathrm{mN}$, with specific impulse values around 300s. The arcjet passes an electric current through a gas propellant, and can either use a nozzle or a magnetic field, at which point it would be categorized as an electromagnetic thruster. Arcjet thrust levels are around $250 \mathrm{mN}$, with 600 s specific impulse measurements.

The resistojet and arcjet require approximately 500W and 2kW of power onboard respectively [6].

\subsubsection{Electrostatic Thrusters}

Electrostatic propulsion operates by accelerating charged particles by electric and magnetic fields. Three types of electrostatic propulsion systems are: 1) electron bombardment thrusters, 2) ion contact thrusters, and 3) field emission thrusters. The electron bombardment thruster bombards a monatomic gas or vapor with electrons to produce positive ions. Ion contact thrusters produce positive ions by passing the propellant vapor through a $1100^{\circ} \mathrm{C}$ porous tungsten contact ionizer. The field emission thrusters produce positive or negative ions by passing propellant droplets through an intense electric field discharge.

Electric thrusters aim to be energy efficient by converting energy into charged particles [6]. They typically produce thrust levels less than $1 \mathrm{mN}$, which makes them useful for precision control of satellites [9].

\subsubsection{Electromagnetic Thrusters}

Electromagnetic thrusters accelerate plasma by electric and magnetic fields. Plasma is predominantly electrically neutral, and makes a good electrical conductor. Electrostatic thrusters accelerate charged particles, and electromagnetic thrusters accelerate electrically neutral plasma. Electromagnetic thrusters are pulsed plasma thrusters, ion thrusters, and Hall thrusters. Pulsed plasma thrusters (PPT) use a Faraday accelerator concept to accelerate the plasma. The plasma is accelerated by passing a current in the direction of an applied electric field, perpendicular to the magnetic field [6]. PPTs have 
specific impulse values around 1000s [9]. Ion thrusters ionize large portions of propellant and then electrostatically extract ions from the propellant using biased grids and accelerate them to high velocities. Ion thrusters have high specific impulses from 2000s to 10,000s [9]. The Hall thruster requires low plasma densities and a high electric field to be effective since collisions must remain low for proper operation. This is due to the crossed electric and magnetic field, and the chance for shorting to occur in the electric field [6]. Thrust output for the Hall thruster is higher than the ion thruster. Hall thrusters typically achieve specific impulses of 2000s [9].

\subsubsection{MEMS-Based Thrusters}

MEMS thrusters are generally categorized as micro rockets. Micro fabrication makes the manufacturing of microthrusters possible. Micro rockets are currently a onetime use system where an array of micro rockets is fabricated into the satellite for control. As the rocket fires the solid propellant, that cell is no longer usable. This is the issue with using solid rockets and micro rockets for micro satellites as the amount of movements available is limited to the size of the rocket array since the solid rocket cannot be re-fired once used [10]. The solid propellant inside the chamber of the rocket is electrically ignited by a high resistance component within the chamber. A MEMS microthruster designed by Zhang et al. used gunpowder as the solid propellant and achieved peak thrust values of $0.05-0.34 \mathrm{~N}$ with specific impulse values of 2.68-14.65s [10]. An image of their microthruster is shown below in Figure 1-1.

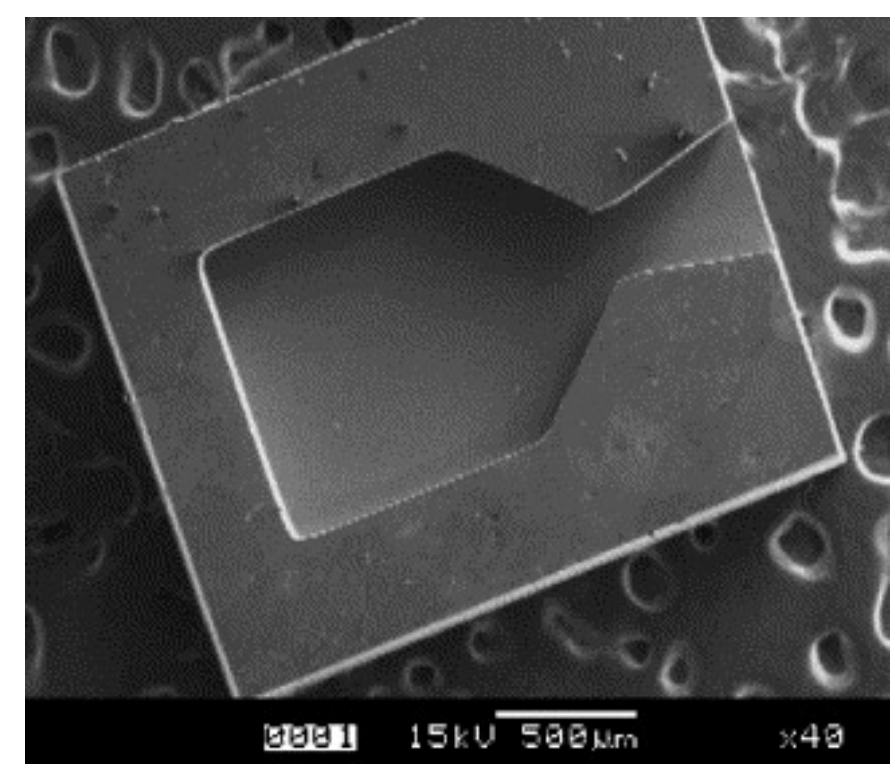

Figure 1-1: MEMS-based Microthruster from Zhang et al 
MEMS microthrusters are well-suited for thrust production for maneuvering, but the specific impulse values show that the effective thrust to propellant weight ratio of this system is not comparable to most electric propulsion systems. Chaalane et al. measured thrust levels of $0.1-1 \mathrm{mN}$, so it is possible to achieve smaller impulse values [11]. These values depend on the size and geometry of the thruster and the propellant choice. The drawback of having unusable weight after the rocket fires is still an issue with these types of thrusters.

\subsubsection{Laser Ablation Microthrusters}

Laser Ablation Propulsion is a relatively new form of micropropulsion which uses energy from the laser light to thermally heat a propellant to a temperature that surpasses its irradiance threshold and converts the thermal energy to kinetic energy via a thermal plasma expansion. For comparison, laser ablation thrusters for satellite applications have generated thrust levels at the $\mathrm{nN}$ level, and have achieved specific impulses of 20,000 s with Aluminum and Carbon propellants. Thrust levels can be dramatically changed depending on the laser parameters and the type of propellant used.

\subsection{Benefits to Laser Ablation Thrusters}

Compared to the other technologies, laser ablation thrusters are capable of a wider range of thrust production. The ability to also use the same system to produce low precision thrust or high thrust values for larger movements is possible. The length of life of a laser propulsion system is greater than MEMS-based systems and comparable to electric propulsion sources. Use of laser propulsion for light craft and satellite transport is also possible using an external independent laser, which makes laser propulsion even more intriguing for its applicability to future systems. One interesting setup has a high power laser in space that would provide energy to surrounding satellites equipped with lenses and propellant, shown below in Figure 1-2 [12]. 


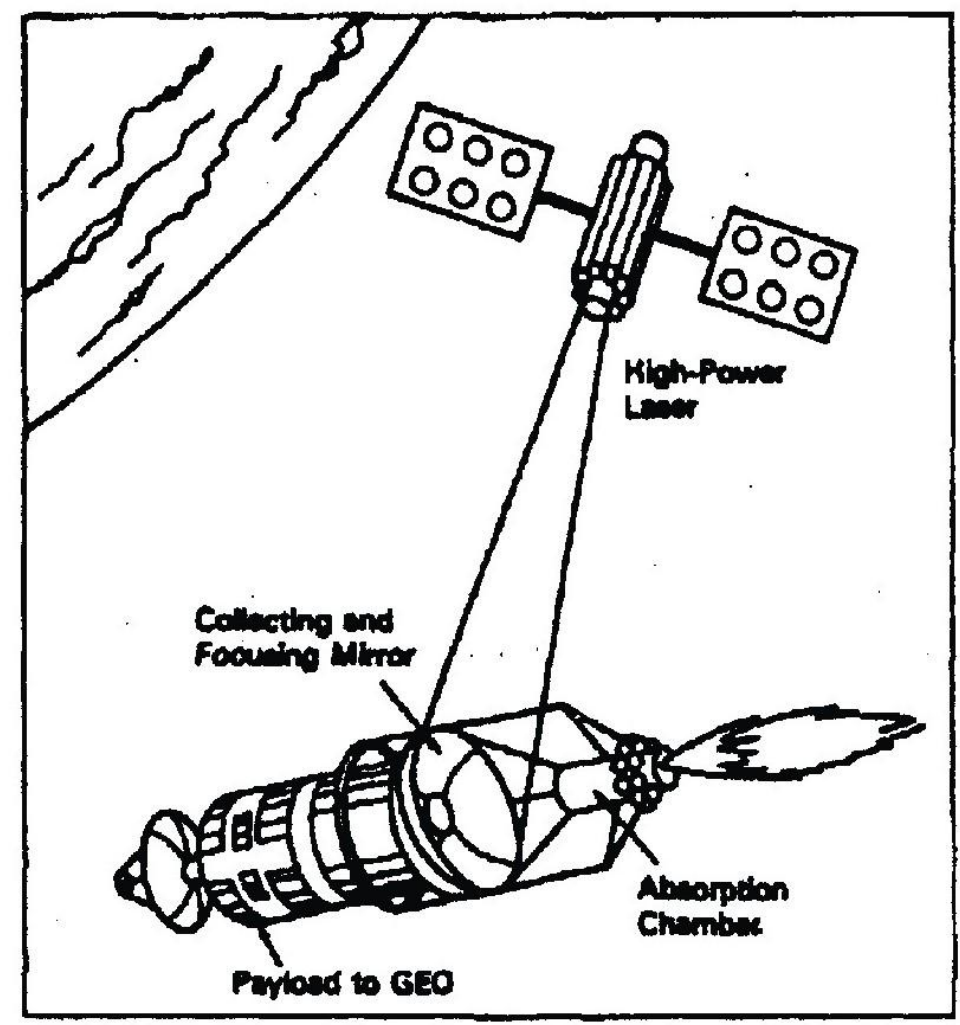

Figure 1-2: Independent Laser Powering Satellite [12]

\subsubsection{Important Laser Parameters for Evaluation}

Laser parameters that are important for propulsion are the pulse width, pulse energy, intensity, and repetition rate, all of which are described later in this report in the Laser Parameters for Propulsion section. Pulse width is the length of the pulse given in seconds, defined by a time-distance relation. The pulse energy defines how much energy is in each pulse, defined by the repetition rate and the average power of the laser. The intensity is important as it essentially defines the ablation potential of the laser with the material. Repetition rate is the rate of pulses exiting the laser cavity, given as a frequency. It defines the amount of time between laser pulses. Repetition rate will increase the average power of the laser, but decrease the pulse energy. Recent studies have used lasers with very low repetition rates due to the high peak power available at lower repetition rates. It is necessary, conversely, to evaluate the effects of laser ablation propulsion at high repetition rates.

\subsubsection{Laser Parameters and Methods for Testing Influence}

For the tests conducted in this work, a 10 Watt diode pumped Nd:YAG laser with dual wavelength; 532 and $1064,20 \mathrm{~ns}$ pulse width, and repetition rate from $10 \mathrm{kHz}$ to $70 \mathrm{kHz}$ is used. The power is variable via 
diode pumping power regulation from $0-10 \mathrm{~W}$, and the repetition rate can be adjusted within the $10 \mathrm{kHz}$ to $70 \mathrm{kHz}$ range. The repetition rate cannot be reduced below $10 \mathrm{kHz}$ as the laser crystal would be in danger of cracking. In order to test for thrust output variations due to these laser parameters, the repetition rate will be tested for each power level as is defined in the Test Apparatus Development section of this report. The setup developed is able to test for thrust variations due to laser power levels, and repetition rate changes. Various groups have developed testing methods that are able to attain fairly accurate results, typically using a pendulum or weight balance system in a vacuum. Another method used by some groups is direct force measurement. These groups also typically use an ICCD camera to capture plasma images to estimate exhaust velocities.

\subsubsection{Experimental Review}

\subsubsection{Luke \& Phipps, 2003}

A setup by Luke \& Phipps incorporates a roll of transparent material with fuel attached that is ablated by the laser to create thrust.

The test apparatus was designed using a torsion pendulum style hanging on a steel wire with a counterweight balancing the system. The entire stand was placed in a vacuum chamber. The amount of torsion induced on the wire was calibrated using a magnetic coil, and the amount of thrust could be measured using a flag pendulum that would catch the plasma shock front. The issue with this method of thrust evaluation is that the flag pendulum is not a reliable or consistent method of measurement as the plume was sometimes not caught by the flag. Their measurements could resolve a $20 \mu \mathrm{N}$ force. The given laser parameters are shown in Table 1-1.

\begin{tabular}{|c|c|c|c|}
\hline $\begin{array}{c}\text { Repetition } \\
\text { Rate }\end{array}$ & Pulse Width & Laser Power & Pulse Energy \\
\hline $20-100 \mathrm{~Hz}$ & $2-10 \mathrm{~ms}$ & $2-14 \mathrm{~W}$ & $75-140 \mathrm{~mJ}$ \\
\hline
\end{tabular}

Table 1-1: Luke \& Phipps 2003 Test Parameters

Thruster performance with these laser parameters is given below in Table 1-2.

\begin{tabular}{|l|c|c|c|}
\hline \multicolumn{1}{|c|}{ Material } & Thrust & $\begin{array}{c}\text { Moment Coupling } \\
\text { Coefficient }\end{array}$ & Specific Impulse \\
\hline PVC & $70 \mu \mathrm{N}$ & $190 \mu \mathrm{N} / \mathrm{W}$ & $220 \mathrm{~s}$ \\
\hline $\begin{array}{l}\text { Kapton with Black } \\
\text { Coating }\end{array}$ & $6.7 \mu \mathrm{N}$ & $26 \mu \mathrm{N} / \mathrm{W}$ & $34 \mathrm{~s}$ \\
\hline
\end{tabular}

Table 1-2: Luke \& Phipps 2003 Test Data 
Luke \& Phipps found that running the laser at longer pulse widths in their system allowed for thrust vectoring as the fuel was being fed perpendicular to the laser beam. They estimated 50 to 60 degrees of thrust vectoring is possible by increasing the pulse width. Thrust vectoring occurs when the laser is used in continuous wave mode, due to the laser ablating faster than the tape can travel, which causes a flame front as shown in Figure 1-3.

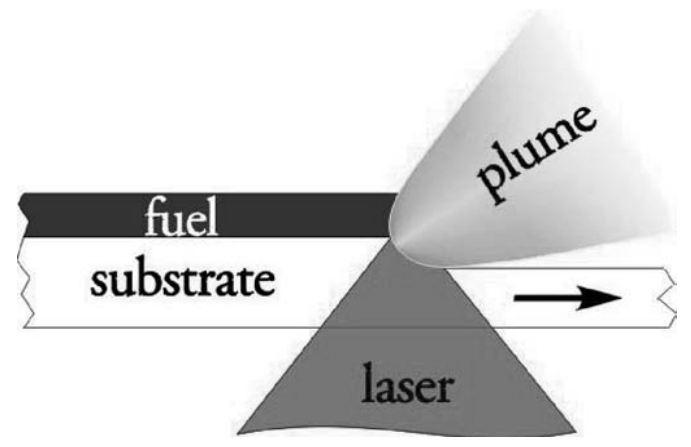

Figure 1-3: Thrust Vectoring [13]

This phenomenon can be prevented by using a pulsed laser, with the option of thrust vectoring if required by altering pulse length. Experimented dimensions of the substrate and fuel are $100 \mu \mathrm{m}$ and $60 \mu \mathrm{m}$ respectively with a width of $1 "$ [14]

\subsubsection{Phipps, Luke, Helgeson, \& Johnson, 2006}

This experiment used a ns pulsed laser with pulse widths of 4-4.55ns, pulse energy of approximately $20 \mu \mathrm{J}$, and spot size of $5-20 \mu \mathrm{m}$. The laser was fired at a gold coated spinning hard drive disk at $8 \mathrm{kHz}$. The test parameters are very similar to those used in this thesis, however the repetition rate is used to be able to fire quickly enough to ablate the spinning disk. An interesting result from these tests is the differing specific impulse values, shown in Table 1-3 below for an aluminum coating. Specific impulse is defined as the ratio of impulse generated to the weight of the ablated propellant per pulse.

\begin{tabular}{|c|c|c|c|c|}
\hline Intensity & Thrust & $\mathrm{Cm}$ & Isp from CmQ* & Isp from TOF \\
\hline $76 \mathrm{TW} / \mathrm{m}^{2}$ & $0.94 \mu \mathrm{N}$ & $111 \mu \mathrm{N} / \mathrm{W}$ & $1120 \mathrm{~s}$ & $6610 \mathrm{~s}$ \\
\hline
\end{tabular}

Table 1-3: Phipps, Luke, Helgeson, Jonhson, 2006 Test Data

This data demonstrates the difference in the specific impulse using different calculation methods [15]. The data will be compared to those calculated in this thesis as the laser parameters are similar. 


\subsubsection{Horisawa \& Kimura, 2002}

In Fundamental Study of Laser Plasma Accelerator for Propulsion Applications, Horisawa \& Kimura used a pendulum system to measure thrust. The pendulum held a sample at the bottom that was ablated by the laser. As the pendulum was displaced, another low power laser reflected off of the sample onto a surface that showed the amount the pendulum displaced. They used thin enough samples to test for transmission mode ablation propulsion. They noticed that some of their values were very low, and that a crater was forming on the front implying that a plume was generating on both sides essentially equalizing the thrust on both sides [16]. They noticed low thrust values with low absorption rate materials and low incident power. When the material was doped to increase the absorption coefficient, the thrust values went up by an order of magnitude [16]. Data from their tests are given below in Table 1-4 [16].

\begin{tabular}{|c|c|c|c|}
\hline Pulse Energy & Fluence & $\begin{array}{c}\text { Moment Coupling } \\
\text { Coefficient }\end{array}$ & Impulse Bit \\
\hline $1.5-2.5 \mathrm{~J} /$ pulse & $0.7-1.5 \mathrm{~kJ} / \mathrm{cm}^{2}$ & $0.2-0.65 \mu \mathrm{N} / \mathrm{W}$ & $0.5-1 \mu \mathrm{Ns}$ \\
\hline
\end{tabular}

Table 1-4: Horisawa, Kimura, 2002 Test Data

Fluence is the amount of energy per unit area of the laser beam, defined as the pulse energy divided by the focal spot cross sectional area. An impulse bit is defined as the smallest amount of impulse possible from a propulsion system. This is an important quantity for micropropulsion systems that require precise movements.

\subsubsection{Pakhomov, Gregory \& Thompson, 2002}

Pakhomov et al. demonstrated a time of flight (TOF) measurement [17]. They proposed that short pulse widths below 100ps should be used for ablative laser propulsion, due to the theory that the ablation duration and plasma lifetime are around 1 $\mu \mathrm{s}$ [17]. From their experiments they stated that high density plasma must be developed in order for ablation to be the main contributor for momentum transfer, which implies that laser pulse duration should be set by the amount of time required for high density plasma to develop. Their given laser parameters are shown in Table 1-5.

\begin{tabular}{|c|c|c|c|c|c|}
\hline $\begin{array}{c}\text { Light } \\
\text { Wavelength }\end{array}$ & Repetition Rate & Pulse Width & Pulse Energy & Atmosphere & Intensity \\
\hline $532 \mathrm{~nm}$ & $10 \mathrm{~Hz}$ & $100 \mathrm{ps}$ & $35 \mathrm{~mJ}$ & $3 \times 10^{-3} \mathrm{torr}$ & $3 \times 10^{13} \mathrm{~W} / \mathrm{cm}^{2}$ \\
\hline
\end{tabular}

Table 1-5: Pakhomov, Gregory, Thompson 2002 Test Parameters 
The TOF measurement was done by placing two copper plates downstream of the ablation both connected to an oscilloscope. They could then determine approximate particle velocity as the particles from the wave front hit both plates with the time difference between successive impacts. Using this method requires a maintained vacuum to allow the particles to travel relatively uninterrupted. Their goal was to determine accurate specific impulse values and compare those to their other works, as well as other published data. They found that specific impulse values decreased gradually with consecutive pulses fired at a lead target. They also found that specific impulses were higher for lighter atomic mass unit propellants. They noted that with a focal distance change from $20 \mathrm{~mm}-50 \mathrm{~mm}$ there was no significant change in specific impulse. Data from their testing included aluminum having an impulse of $300 \mathrm{nNs}$, with a mass material removal rate of $0.2-0.3 \mu \mathrm{g} /$ pulse. Carbon had mass removal rates of approximately $0.15 \mu \mathrm{g} /$ pulse. Copper samples reached $55 \mathrm{~km} / \mathrm{s}$, and a very high 20000 s specific impulse was recorded for Carbon samples [17].

\subsubsection{Pakhomov, Thompson, Swift \& Gregory, 2002}

Pakhomov et al. [18] in another experiment used a direct force measurement piezoelectric sensor made by PCB Piezotronics, Inc. The sample was placed directly on top of the sensor and was fired at using a similar laser used as that described in Table 1-5. The pulse energy was reduced to $8 \mathrm{~mJ}$ in these tests. They found that the material removal rate was twice as high in vacuum than in atmospheric conditions, due to material deposition from atmospheric pressure [18]. They also found that the material removal rate was generally lower for lighter atomic mass propellants. For the metals they tested, they found moment coupling coefficients in the range of $20-80 \mu \mathrm{N} / \mathrm{W}$ [18]. The moment coupling coefficient $\left(\mathrm{C}_{\mathrm{m}}\right)$ is the ratio of impulse to laser pulse energy, per pulse, for ablation of the propellant.

\subsubsection{Maesato, Koizumi, \& Tahara, 2006}

In Performance Characteristics of Low-Power Laser Ablative Thrusters for Small Satellites, an Nd:YAG laser with wavelength of $1064 \mathrm{~nm}$, pulse width of $6 \mathrm{~ns}$, and an output energy of $0.65 \mathrm{~J}$ is used to generate thrust from various polymers. The testing was conducted at $10^{-3}$ torr using a pendulum system with an electromagnetic damper and counterweight balance. Their testing results of the polymers are given below in Table 1-6. 


\begin{tabular}{|c|c|c|}
\hline Material & $\begin{array}{c}\text { Moment Coupling } \\
\text { Coefficient }\end{array}$ & Specific Impulse \\
\hline PTFE (10\% carbon) & $100 \mu \mathrm{N} / \mathrm{W}$ & $170 \mathrm{~s}$ \\
\hline POM (20\% carbon) & $42 \mu \mathrm{N} / \mathrm{W}$ & $300 \mathrm{~s}$ \\
\hline
\end{tabular}

Table 1-6: Maesato, Koizumi, \& Tahara, 2006 Test Data

Maesato et al. noted that after the first shot to the polymers the moment coupling coefficient decreased rapidly with each successive shot. They proposed that the laser pulse increased the porosity of the material, and in turn reduced the absorption efficiency of the laser pulse to the solid propellant [19].

\subsubsection{Yokoyama, Horisawa, Funaki, Kuninaka, 2007}

In Fundamental Study of Laser Micro Propulsion Using Powdered-Propellant, an investigation on the thrust production with a powdered propellant was conducted. In the tests a pulsed Nd:YAG laser with a wavelength of $1064 \mathrm{~nm}$, pulse width of $10 \mathrm{~ns}$, and pulse energy of $400 \mathrm{~mJ}$ was used. The tests were conducted in a vacuum chamber at $10^{-3}$ torr and used a pendulum mass type target with a displacement sensor behind the sample area. They used the plasma plume to imply a force on the sample area; the data is given below in Table 1-7 [20].

\begin{tabular}{|l|c|c|}
\hline \multicolumn{1}{|c|}{ Material } & Impuse Bit & Specific Impulse \\
\hline Silicon Powder & $7 \mu \mathrm{Ns}$ & $4 \mathrm{~s}$ \\
\hline Toner Powder & $5-15 \mu \mathrm{Ns}$ & $3-5 \mathrm{~s}$ \\
\hline $\begin{array}{l}\text { Carbon Particle } 500 \mu \mathrm{m} \\
\text { diameter on cellophane Tape }\end{array}$ & $2-4 \mu \mathrm{Ns}$ & $1-3 \mathrm{~s}$ \\
\hline $\begin{array}{l}\text { Carbon Particle } 500 \mu \mathrm{m} \text { behind } \\
\text { glass }\end{array}$ & $6-18 \mu \mathrm{Ns}$ & $7-18 \mathrm{~s}$ \\
\hline
\end{tabular}

Table 1-7: Yokoyama, Horisawa, Funaki, \& Kuninaka, 2007 Test Data

The data ranges are due to pulse energy changes. It is noted that the cellophane tape experiment absorbs laser energy but does not contribute to thrust and should be avoided. Using a transparent substrate may be the only means to holding a sample, but the purpose should obviously be very significant if thrust generation is being sacrificed.

These testing methods are all good options to evaluate various thrust parameters. Use of a direct force measurement method is thought to be the best option but is typically costly. Using the method outlined 
in this report has been very cost effective, and appropriate results compared to those published have been gathered from testing.

\subsection{New Trends in Micropropulsion Research}

New trends in research for laser micropropulsion and for laser propulsion are focused on shorter pulse widths and more energetic material compositions. Using femtosecond laser pulse widths has reduced the plasma pulse energy absorption due to the pulse fully entering the material before the plasma plume is generated. As laser technology advances, higher power lasers are available and are being used to conduct experiments as well. Cai et al. found that irregularly shaped holes from ablation occurred due to their lower power laser. They propose that an inhomogeneous energy distribution is attributed to intensities that are too low for the material's ablation [21]. For satellite use, higher power diode lasers are being tested with various materials for systems that would require the laser to be onboard the craft. Investigations on the use of nozzles and magnetic fields are also ongoing to increase the specific impulse of the propulsion system.

Other research on light craft where the laser source would be independent of the craft is also being conducted for various purposes. Higher power lasers could be used to transport satellites and craft to space, but would be left on earth or would already be in space due to their weight and size.

\subsection{Objectives of This Work}

High repetition rate $\mathrm{kHz}$ Nanosecond lasers have not been extensively studied for laser propulsion. The objective of this work is to test kHz nanosecond laser parameters' effects on micropropulsion by altering the repetition rate and laser power during testing. The ability to test a new range of parameters for laser micropropulsion is a very beneficial contribution to this new field. Along with these tests several materials will be tested and the damaging effects the laser power and repetition rate have on the material will be evaluated due to the typical assumption that all propellant is usable for the propulsion system.

During the design of the testing apparatus, it is required to develop a sensor system. So as another contribution to this thesis a new method for testing micropropulsion using piezoelectric sensors will be developed and tested. 


\section{Chapter 2: Laser Background and Principles}

The applicability of the laser has been constantly growing over the last half century. Within the last 20 years different chemical compounds for various types of lasers have been discovered, and their potential for power output has also increased. New methods for light intensity growth, along with higher repetition rates, have given the laser greater ability to be used for new objectives.

Lasers have great applicability for thruster use. The efficient conversion of electrical energy to thrust is what is sought after in propulsion applications. The manufacturing industry also benefits greatly from laser ablation, as certain materials are not easily drilled by normal methods. For instance, drilling through rubber or diamond is very difficult without the use of a laser, which can cut through these materials very easily [22].

Having a basic understanding of how the laser functions, and the different methods available to increase beam power are important to continue with research of a laser ablation topic. This section will focus on the laser cavity and its components, how the cavity intensifies the beam, and how the beam can then be focused to a spot. Different types of lasers will also be discussed, along with their common applications.

\subsection{Laser Operation}

The laser consists of several main components that intensify light via a source, also called a pump. The pump stimulates atoms in the parent medium. Light intensity will continue to grow within the laser cavity, reflecting axially between mirrors on both sides of the medium [23]. Light intensity growth occurs by stimulated emission. When energetic light is directed on a material, there is a transition of electrons from occupied spaces below Fermi energy to unoccupied spaces above Fermi energy [24]. As electrons decay to their lower energy levels they emit photons, which then stimulate other atoms within the laser cavity [22]. This well describes the acronym, LASER: Light Amplification by Stimulated Emission of Radiation.

The energy that is released as light during stimulation comes from a phenomenon known as population inversion. Laser operation not only consists of stimulating light emission, but also intensifying it by inducing coherence using methods such as Q-switching, and mode locking. The average power of a laser is the beam area multiplied by the laser intensity [25]. 


\subsubsection{Coherence}

Coherence is achieved when the laser is oscillating in one mode, implying that any two points along the beam will constantly be in phase [26]. Having the beam oscillate in one mode provides more power than there would be if the beam were oscillating in multiple modes. When multiple modes are present, the interference caused by the waves overlapping can cause dark spots in the material the laser beam is being shot at [27]. Dark spots imply low power regions of the beams cross section.

Coherence length and time are important characteristics of the laser beam. The coherence time is the amount of time the laser beam can travel before it loses phase continuity, and the coherence length is the distance the beam can travel as a result [27].

\subsubsection{Q-Switching and Mode Locking}

Q-switching greatly enhances the applicability of the laser by allowing the intensity to grow within the laser cavity before releasing the beam, which is called a Pulsed Laser. During the process, a DC voltage is applied, rotating the polarization of the light by $90^{\circ}$ [23]. A polarizer placed before the front mirror reflects the light back towards the back mirror, where it could also then be mode locked. Mode locking happens by bouncing the light off of a back mirror where gain modulation attempts to lock the waves into a single mode by changing their phase. The light will continue to travel axially within the cavity while stimulating other atoms and gaining intensity. Once the DC pulse stops polarizing the light, the laser beam exits the cavity [23].

\subsubsection{Monochromaticity}

Monochromatic light means that only a single line in the light spectrum was emitted by the laser cavity [28], implying that the laser only emits a single wavelength [27]. However, it is fairly implausible, and in a realistic sense monochromatic light is impossible [26].

\subsection{Types of Lasers}

The pulsed laser was briefly described in the previous section, but they will be discussed here for the sake of comparison with a continuous wave (CW) laser. Several different types of lasers with respect to chemical composition and state will also be discussed.

\subsubsection{Pulsed Laser}

A pulsed laser emits pulses of beamed light at a rate typically determined by a function generator, and a pulse width which can be regulated by Q-switching. The Nd-YAG laser (Yttrium Aluminum Garnet doped with Neodymium) is an example of a solid state pulsed laser, which can emit a laser power greater than 
a MW in nanosecond pulses. If the same laser is used in continuous wave mode, powers slightly more than $10 \mathrm{~W}$ are attainable [23]. This shows that the pulsed laser can have a much higher peak power than the continuous wave laser [28].

\subsubsection{Femtosecond and Nanosecond Pulsed Lasers}

Pulsed lasers have several main components which transfer the energy of the beam and amplify it to levels that the oscillator could not safely do on its own. The oscillator produces regular low energy short pulses that are sent to a stretcher, which then converts the femtosecond pulse into a 50-200ps chirped pulse [29]. A chirped pulse is one where the instantaneous pulse is red in the front and blue at the back [30]. The wave then passes through several amplification stages to increase its pulse energy by a factor of $10^{7}-10^{9}$. A compressor then works to transmit the amplified pulse of the same duration that the oscillator output. Chirped Pulse Amplification (CPA), as shown below in Figure 2-1, is a method to ensure the components remain undamaged, as they would be damaged if the oscillator output that much energy to the amplifier [29].

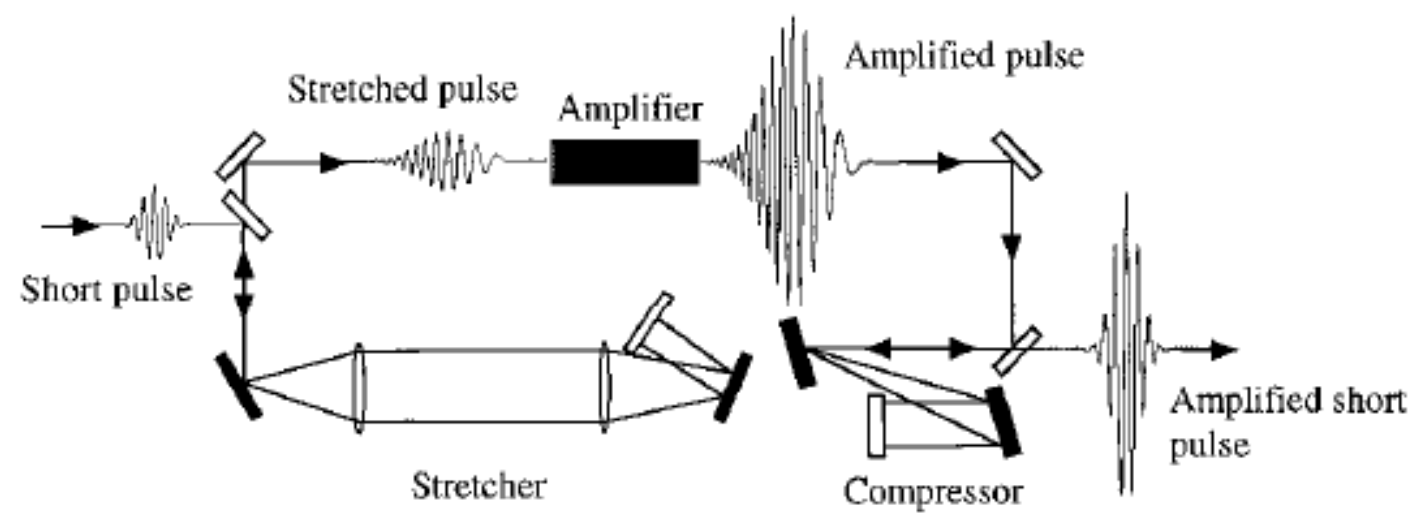

Figure 2-1: Chirped Pulse Amplification Operation Diagram [31]

Pulse duration is inversely proportional to the bandwidth of the pulse, which implies that a large bandwidth is required to create a short pulse. Femtosecond lasers are typically Ti-Sapphire due to its large bandwidth, high damage threshold, and saturation fluence of $1\left[\mathrm{~J} / \mathrm{cm}^{2}\right]$. A femtosecond pulse typically contains a few nanojoules of energy, which can be amplified by quite a bit with use of diffraction gratings. Diffraction gratings "stretch" the pulse by a factor of $10^{3}$ or $10^{4}$, and impose a positive chirp so the longer wavelengths emerge before the shorter ones [29]. Large power lasers are currently in existence, and larger ones are being manufactured. With laser powers exceeding a petawatt, the amount of energy available can easily damage the laser components. 
The safest way to increase power without damaging these components is to further shorten the pulse length [29]. This will allow a greater amount of output energy from a relatively low amount of energy input.

\subsubsection{Femtosecond and Nanosecond Ablation}

Within the ablation process, heat conduction away from the affected zone is an issue. The laser heating source term, $\mathrm{S}$, is given in Equation (2.1) below [32]. The equation describes how the laser intensity and absorption coefficient directly relate to the material heating.

$$
S=I(t) A \alpha \exp (-\alpha z)
$$

Where $I(t)$ is the intensity of the laser spot, $\alpha$ is the material absorption coefficient, and $A$ is the surface absorptivity defined by Equation (2.2) below, where $\mathrm{R}$ is the surface reflectivity [33].

$$
A=1-R
$$

For ultra short laser pulses, often defined by pulses shorter than $100 \mathrm{ps}$, the pulse is too short to allow for heat conduction to the surrounding zone [34]. This is due to the pulse fully entering the material before the electrons and ions are able to equalize, called the electron-ion relaxation time. Due to the pulse penetrating the material before the ablation begins [35], the electrons reach very high energies, and the lattice and ion energies are much lower than that of the electrons [36]. The subsequent electron-ion energy transfer very quickly heats the material to a very high temperature, much higher than that of longer pulses. The material quickly vaporizes, but the heat affected volume is much smaller with femtosecond laser pulses [36]. This reduces the amount of energy lost within the ablation process, and also gets rid of the surrounding zone being damaged by the laser pulse. For manufacturing purposes, it is very important to reduce damaged zones by heat. For propulsion purposes, it is not as much of an issue to have a heat affected zone. However, it could potentially damage the propellant or create some porosity. Nanosecond pulsed lasers are considered too short to bypass the surrounding zone being affected by heat conduction. Often nanosecond pulsed lasers are partially absorbed by the expanding plume, which is considered laser supported detonation (LSD).

Nanosecond pulsed diode pumped fiber laser amplifiers are capable of efficiencies greater than $40 \%$ and are low mass devices, which make them very applicable for micro propulsion use [7]. Fiber lasers currently are capable of delivering $25 \mathrm{~kW}$ peak power, with $10 \mathrm{~ns}$ pulse widths at $100 \mathrm{kHz}$, delivering an 
average power of 25W [37]. Microchip lasers on the other hand have efficiencies of $1-2 \%$ because most of the power available is required for chip cooling [7].

\subsubsection{Continuous Wave Laser}

A continuous wave laser is constantly under steady pumping, outputting a continuous beam with no breaks [23]. The output power that results is less due to the inability for the intensity to grow very much before the beam exits the laser cavity. The decay rate is such that the laser can maintain a lower power beam than if it were pulsed.

\subsubsection{Solid-State Lasers}

Solid-state lasers consist of a transparent crystal or glass rod with the ability to remove unwanted heat, and can undergo stimulated emission. The host materials are the majority of the rod, but the active substance in the material is typically $50 \%$ of the material composition, this is also called the dopant [22]. Some doping substances used are Chromium, Uranium, and Neodymium. Solid state lasers use a flash lamp or a diode as a pumping source with a reflective inner cavity, which encounters significant losses [23].

\subsubsection{Ruby Laser}

The Single Crystal Ruby laser is no longer a widely used solid state laser. This is due to the low overall system efficiency. The laser requires a large amount of pumping to initiate population inversion and in turn activate stimulation [38]. It's comprised of Aluminum Oxide as the host doped with Chromium atoms. The Chromium atoms emit red light when stimulated by green light [22]. The Ruby laser typically emits a light wavelength of $694 \mathrm{~nm}$.

\subsubsection{Nd:YAG}

The neodymium doped yttrium aluminum garnet laser can emit light wavelengths of $1.06 \mu \mathrm{m}$ to $1.32 \mu \mathrm{m}[28] . \mathrm{Nd}: Y A G$ is the most commonly used laser due to its high gain capabilities. Neodymium doped lasers are good for diode use as they have a high upper level lifetime, requiring less power from the pumping source [38].

\subsubsection{Nd:YVO}

Neodymium doped Yttrium Orthovanadate is often used for diode and microchip lasers. The material has strong absorption abilities at a wavelength of $809 \mathrm{~nm}$ [38]. The material is good for diodes due to the higher efficiency, and low power requirement from the pumping source [38]. 
This is due to the fact that diode laser systems typically do not have much pumping power available.

\subsubsection{Liquid Lasers}

Liquid and dye lasers are not very common. The dye laser is comprised of organic substances as the active ingredient; water, ethanol, benzene, methanol, acetone etc. [27]. The liquid laser and the solidstate laser operate in a similar manner.

\subsubsection{Gas Laser}

Gas lasers briefly described in this section are classified by their mediums; atomic lasers, ion lasers, molecular lasers, and excimer lasers.

\subsubsection{Atomic Lasers}

He-Ne gas laser is an example of an atomic laser with a wavelength of $633 \mathrm{~nm}$. It is small, cheap and, as a result, is usually used for optical experiments, measurements, or display purposes [28]. Argon lasers have the ability to retain a coherent beam that is more powerful than the He- $\mathrm{Ne}$ beam, and can attain wavelengths of visible light from light green to deep blue [23].

\subsubsection{Molecular Lasers}

$\mathrm{N}_{2}$ is an example of a molecular laser, which emits ultraviolet light at a wavelength of $337 \mathrm{~nm}$. These lasers are typically used for infrared research [28].

\subsubsection{Excimer Lasers}

$\mathrm{Xe}_{2}$ is an excimer gas laser emitting a light wavelength of $173 \mathrm{~nm}$. These are typically used for photochemical research [28].

\subsubsection{Semiconductor Lasers}

Optoelectronic applications and micro electronics benefit from the semiconductor laser, which typically has dimensions of $1 \mathrm{~mm} \times 1 \mathrm{~mm} \times 1 \mu \mathrm{m}$. Semiconductor host materials include Germanium, and Silicon. The semiconductor laser uses dopants called $\mathrm{p}$-type, $\mathrm{n}$-type, and $\mathrm{p}$-n-type. Semiconductor lasers can also be pumped by another laser, often a crystal diode laser [27].

\subsection{Diode Lasers}

The diode laser is a very small, low power laser that has many applications such as laser ablation propulsion on a satellite, laser pointers, CD and DVD players, etc. They are typically used when weight, size, and power availability are critical. In the past, the low power output of most diode lasers generally 
did not allow them to ablate metals, which limited their ablation uses. If used for propulsion, polymers were used as the propellant due to the fluence produced by low power, long pulse width lasers. Recent advancements in diode laser technology have boosted peak power and reducing pulse width, increasing their usability for micropropulsion. Using a nanosecond pulsed laser instead of a ms pulsed laser would require approximately 500 times less fluence on the target, which implies both a larger distance allowable between the lens and the target and a lower power laser can be used [39]. For micropropulsion, diode lasers and fiber lasers are essential due to their compactness. Light weight diode lasers are able to deliver sufficient power to ablate most propellants to generate thrust requirements for precision maneuvering on nano-satellites. Diode technology for micropropulsion is described later in this section.

Other applications of diode lasers are for pumping sources. Nd-YAG microchip lasers have been pumped using diode lasers, which then could output high enough power levels to ablate metals [40]. Diode pumped lasers are able to achieve repetition rates of 1 to 2 magnitudes higher than flash lamp pumped lasers due to their reduced heating of the laser gain medium [41].

Laser ablation requires that the binding energy of the material be surpassed by the laser beam energy. Typically for laser cutting, or laser ablation processes, a pulsed laser will be used to attain high enough energy densities [40]. Diode lasers are not typically capable of achieving these intensity levels. For laser micropropulsion, the system would use a diode laser as an energy source due to the low weight requirements of the satellite.

Recent uses of diode lasers also include power beaming. Power beaming uses high power lasers to transfer power from one location to another. This is useful for applications where power lines are not possible or feasible, for instance, a moving object with an electric motor. The laser energy charges batteries onboard the device, and the batteries power an electric motor. The laser could also power the object directly. Recent advancements in diode technology have made using diode lasers more applicable for a space elevator design. Amplified power output of over $3.5 \mathrm{~kW}$ is possible using stacked diode arrays [42]. Another use of a stacked diode array was used as a diode pumping source for a terawatt laser developed by Siebold et al. that outputs $85 \mathrm{fs}$ length pulses [43]. A laser with this power level capability could be used to provide propulsion to a craft or satellite hundreds of kilometers away.

\subsubsection{Wavelengths of Diode Lasers}

Laser wavelength is an important factor for propellant material choices and diode types. There are many different lasing materials that can be used in the diodes, so propellants are not limited to the 
wavelength of the specific diode. Wavelengths of $447 \mathrm{~nm}$ to $900 \mathrm{~nm}$ are possible by changing the diode material. For instance, a Zinc Selenide diode would produce a $447 \mathrm{~nm}$ wavelength light, and a Gallium Arsenide diode laser would produce a 900nm wavelength light [3]. Diodes can also use frequency doubling to reduce the wavelength if required, but can create inefficiencies if not focused to high intensities [3].

\subsubsection{Diode Lasers for Micropropulsion}

Diode lasers are useful for satellite applications because they are capable of being made very small and lightweight. This is due to the high gain materials used for diode lasers, which allow them to have smaller than normal cavities being around $300-500 \mu m$ long [3]. Having a smaller laser allows for a lighter satellite that reduces launch costs and propellant requirements as well. Diode lasers are also known for their high efficiency which reduces energy requirements. Energy storage for communications could be shared with the energy storage for the laser and could thus reduce weight even further. Diode lasers with output powers of $1[\mathrm{~W}]$ or greater typically have efficiencies of approximately $50 \%$ [40].

Diode lasers are also capable of being used in conjunction with one another to increase power output. Being able to use multiple smaller, lower power, lasers together to create one output beam can be beneficial to instances that require higher thrust output but still require a low weight design. Luke et al replaced a single laser with four fiber coupled lasers capable of an output power of 14[W] pulses [14]. Laser beam width contributes greatly to the energy density potential with the use of lenses to focus on a single spot. If the output power of the laser is increased by increasing the width of the cavity, then more energy can be focused on a spot. The problem with diode lasers is that if the cavity width is increased too much then internal cross lasing can occur within the structure due to the diode sidewalls. Typically, emission widths on diodes are $75-600[\mu \mathrm{m}]$, but with reduced diode sidewalls, emission widths as large as $1500[\mu \mathrm{m}]$ have been made [44].

Recently, methods to increase power output and reduce pulse width for diode lasers have been researched. Ulm et al. developed a master oscillator power amplifier system for a 920nm wavelength to achieve pulse widths as short as $267 \mathrm{fs}$ with an average power of $708 \mathrm{~mW}$ and a peak power of $661 \mathrm{~W}$, at a repetition rate of $3.3 \mathrm{GHz}$ and pulse energy of 213pJ [45]. This was done by compressing picoseconds pulses that are output from an oscillator to femtosecond pulses post amplifier. High power diode lasers are also being developed for many applications. Fiebig et al. developed a distributed Bragg reflector tapered diode laser to output $12 \mathrm{~W}$ of optical power, at $44 \%$ conversion efficiency. The diode laser outputs a wavelength of $979 \mathrm{~nm}$ [46]. The benefit to the design by Fiebig et al. is that it retains its 
compactness, while many other developments have increased in size and weight. Power conversion efficiency (PCE) is a highly sought after parameter for researchers developing diode lasers. The efficient conversion of electrical energy to light energy can enable higher power lasers to be designed and used for applications due to the lower amount of energy required for cooling and pumping. Cai and Kanskar have developed a continuous wave (CW) laser that has a PCE of 67\%. The laser operates at 1060nm, and has a steady heat sink temperature of $25^{\circ} \mathrm{C}$, which shows that it is a stable design. They used an aluminum free material (GaAs) system which they state reduced the facet temperature rise by an order of magnitude when compared to diode systems using AlGaAs [47]. 


\section{Chapter 3: Fundamentals of Laser Ablation Propulsion}

Laser ablation of a material can differ greatly depending on the following laser pulse parameters; pulse duration, pulse frequency, pulse energy, and wavelength. For example, long pulse ablation involves evaporating the material while short pulse ablation is governed by hydrodynamic motion [48].

Hydrodynamic motion is caused by thermal expansion of the material while energy from the laser is being absorbed. When hydrodynamic motion begins it creates a plume and a crater, which both expand. This is a phase transfer of the solid directly into hot, radiant, expanding plasma [18]. The temperature distribution within the material is non uniform at the beginning of the hydrodynamic motion, and the temperature of the material decreases with depth due to conduction losses [48].

Laser ablation for nanosecond or longer pulses generally occurs in three stages [49]. The first stage is the solid material absorbing the laser beam. This causes a large amount of heating due to the amount of energy being irradiated on the material. Stage two involves material being ejected while continuing to absorb energy from the laser. This stage contains laser-gas and laser-plasma interactions as the material is being ejected. The ejected matter is known as plasma. Femtosecond laser pulses do not have this stage while interacting with material since the pulse is so quick that all material is ejected as plasma after the pulse has finished being absorbed [49][50]. Stage three begins when the pulse interaction ends. During this stage a plume expands adiabatically from the surface of the material. The plume is often used for many different purposes. It can be used to place a thin film of material on a body opposite the material being ablated, called sputtering. It could also be used for micro propulsion to produce thrust necessary for satellite maneuvering.

The laser energy absorbed in stage one is by electrons transferring heat through cold material with conduction. There is an exchange of energy between the electrons and the lattice of the material. During the hydrodynamic stage when material removal begins, the electron and lattice temperatures are approximately the same [48]. The expanding cloud, also known as the plume, has a very thin shell of ejected matter on its external edge. Gas-liquid phase material fills the shell, and the density of this material phase decreases as the plume expands proportionally to the time of expansion. 


\subsection{Laser Parameters for Propulsion}

Laser propulsion requires high intensity laser pulses for propellant ablation. Laser-matter interactions differ greatly with different materials. For example, transparent materials transport light at low intensities, but absorb and heat very quickly at high intensities [33]. This explains why a laser can be directed and focused through a series of lenses at a wider beam diameter, however at the focal point the intensity greatly increases and has a higher potential for ablation.

In order to get high intensity pulses either a high power laser must be used, or a shorter pulse width can be used with a lower power laser, possibly to decrease the size, weight, and cost of the laser. The average power of a laser is defined by Equation (3.1) below given in Joules per second, or Watts [W].

$$
\text { Average Power }=\frac{\Delta \text { Energy }}{\Delta \text { time }}
$$

Then using the average power, the energy per pulse can be calculated. The energy per pulse is an important value as it directly influences the intensity of the laser, shown in Equation (3.2) defined as joules of energy per pulse [J].

$$
E_{\text {pulse }}=\frac{\text { Average Power }}{\text { Repetition Rate }}
$$

The repetition rate is the frequency that the laser releases a pulse. The fluence of the laser is defined as the energy per cross sectional area of the laser at its focal point, or spot $\left[\mathrm{J} / \mathrm{cm}^{2}\right]$.

$$
\text { Fluence }=\frac{E_{\text {pulse }}}{\text { Spot Area }}
$$

A useful method to increase laser beam power is to reduce the spot size, which is done with lenses. Spot size (diameter), $w_{0}$, of the focused beam can be calculated using Equation (3.4) [51].

$$
w_{0} \approx \frac{1.27 \lambda f}{D}
$$

Where $f$ is the focal length of the focusing lens used, $\lambda$ is the wavelength of the laser light, and $D$ is the diameter of the incident collimated beam.

Equation (3.5) shows how laser intensity is inversely proportional to the pulse width, defined as Watts per cross sectional area of the spot $\left[\mathrm{W} / \mathrm{cm}^{2}\right]$. 


$$
\text { Intensity }=\frac{\text { Fluence }}{\text { Pulse Width }}
$$

The pulse width is the length of the laser pulse given in seconds. Another important parameter for laser ablation is the peak power of the pulse. Where the energy per pulse may be low due to the repetition rate, Equation (3.6) shows how the pulse width can significantly increase the peak power of the laser pulse. For thrust purposes, peak power is an important parameter due to the material dissipating heat with time. In order to blast material from the zone, a high intensity short pulse is desired to reduce the amount of time available for heat conduction.

$$
\text { Peak Power }=\frac{E_{\text {pulse }}}{\text { Pulse Width }}
$$

With a high peak power from a short pulse width, lower power lasers can be used for propulsion. This opens the field to diode lasers which are much lower power lasers compared to large table-top lasers.

\subsection{Fluence}

Ejected materials by the ablation are strongly influenced by laser energy density [52]. Energy density is also known as fluence, with units typically $\left[\mathrm{J} / \mathrm{cm}^{2}\right]$. Laser fluence is not uniformly distributed over the focal spot diameter, and its typical distribution is given below in Equation (3.7) [48].

$$
F l(r)=F l \exp \left(1-\frac{r^{2}}{w_{0}^{2}}\right)
$$

Where $F l$ is the surface energy density of the absorbed laser $\left[\mathrm{J} / \mathrm{cm}^{2}\right], \mathrm{r}$ is the distance from the center of the laser spot perpendicular to the laser beam, and $w_{0}$ is the spot diameter from Equation (3.4). This non uniform fluence distribution is described below in Figure 3-1 with a Gaussian beam profile.

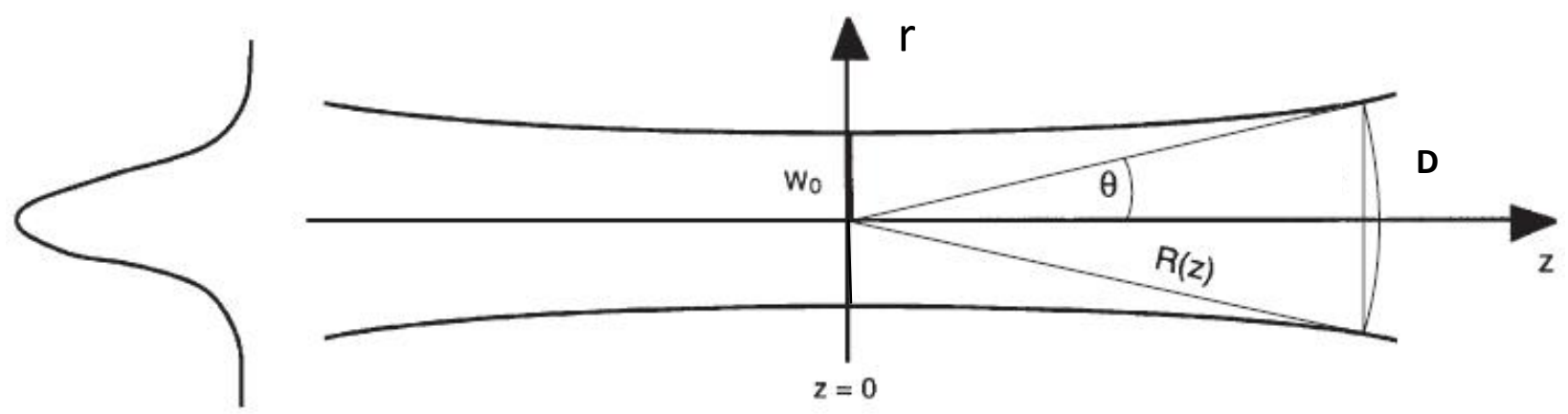

Figure 3-1: Gaussian Beam profile [30] 
Where the $z$ axis is the centerline of the beam, $R(z)$ is the radius of curvature of the wave surface, $D$ is the radius of the collimated beam, and $w_{0}$ is the minimum diameter of the beam being focused by the lens on the right. Along $z$ is the depth of focus (DOF), defined by Equation (3.8).

$$
\begin{aligned}
D O F & =\frac{\pi w_{0}{ }^{2}}{2 \lambda} \\
& \approx 2.5 \lambda\left(\frac{f}{D}\right)^{2}
\end{aligned}
$$

The DOF is the distance that the beam can be considered focused. The exact focal point is at the minimum diameter $w_{0}$, but determined by the wavelength we can assume the beam to be focused at any point within the DOF. The DOF is centered at the focal point and extends $0.5^{*}$ DOF in both directions along $z$.

Figure 3-2 below describes the intensity in a Gaussian beam, where the highest intensity is at the center of the beam spot. [30].

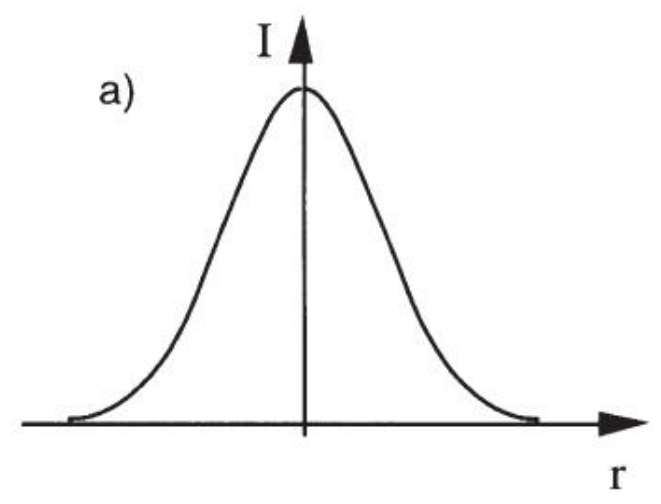

Figure 3-2: Intensity of Gaussian Beam Across Beam Diameter [30]

The fluence threshold $\left(F_{T h}\right)$ of the material must be reached in order for ablation to occur. The fluence threshold basically describes how much energy can be absorbed by the material before it begins to ablate, or the minimum amount of fluence required to ablate the material [48].

The ablation process is most simply described by Equation (3.9): ablation rate per pulse [53].

$$
d(F)=\frac{1}{\alpha_{e f f}} \ln \left(\frac{F l}{F l_{T h}}\right)
$$


Where $d$ describes the ablation rate per pulse, $\alpha_{\text {eff }}$ is the effective absorption coefficient, $F l$ is the irradiation fluence, and $F l_{T h}$ is the ablation fluence threshold of the material.

\subsection{Plasma Plume Expansion}

As energy is focused into the ejected material with high intensity, it breaks down into a ball of expanding plasma [5]. Plasma expansion is a topic of great depth. Groups have researched the effects of plasma expansion on thrust in many different scenarios. Schall et al. suggested using a nozzle to further contribute to thrust generation by using the high pressure expanding gas [5]. The effects of plasma expansion are heavily dependent on laser type, material, atmospheric conditions etc. Ambient air density influences surface pressure during ablation and plume generation [54][50]. However, the initial expansion of the plume is not affected much since the large $1 \mathrm{kbar}$ driving pressure is typically much higher than ambient pressure [50].

Horisawa \& Kimura found that the moment coupling coefficient $\left(C_{m}\right)$ decreased as incident pulse fluence levels increased [16]. Eckel et al supported this theory by finding that the amount of energy that isn't absorbed by the plasma is inversely proportional to the pulse energy [55]. This implies that the amount of energy that actually reaches the target material is a greater percentage of the incident pulse energy when the pulse energy is lower. Plasma further influences the pulse reaching the material depending on the length of the pulse. Since plasma growth times are very short, about 1 to $2.5 \mu \mathrm{s}$, a shorter pulse should be used to reduce plasma and pulse interaction time [56]. Plasma lifetimes are also only several microseconds long [18]. Eckel et al also found that pulse lengths shorter than the plasma build up time of approximately $1 \mu$ s should be used to reduce the amount of energy lost to plasma plume absorption [55]. This is due to a breakdown of the material or air in front of the target which feeds the plasma expansion away from the surface [55]. There is thus a loss of energy deposition and as a result a loss of impulse potential from the interaction.

The combustion effects from testing in air as opposed to in a vacuum do not drastically contribute to thrust production. Sterling et al. found an increase of about $12 \%$ when testing in air vs. testing in 1 atm of nitrogen [57]. A plasma plume example is given below in Figure 3-3 [18]. 


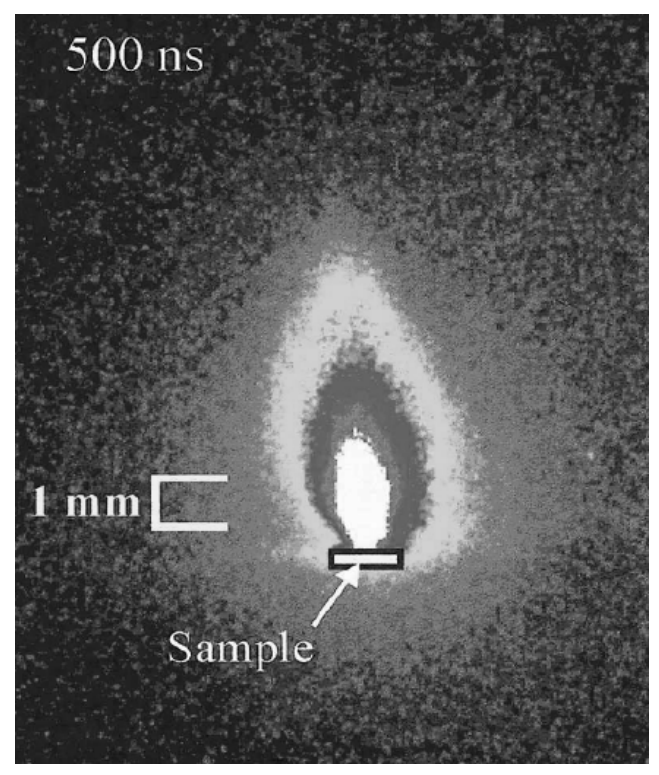

Figure 3-3: Actual plasma plume from lead sample [18]

Eckel et al found that an increase in pulse energy did not linearly increase plasma wave front velocities [55]. Several groups have investigated the effects of constricting the plasma expansion with a thicker medium to increase thrust. Zheng et al used both a glass plate and water in different tests to determine $C_{m}$ value increases [58]. They found an increase in the $C_{m}$ value by 30 times vs. a conventional direct ablation method in atmospheric conditions. Energy conversion from the plasma expansion to kinetic energy in the water is converted much more than in air. The plasma expansion confinement increased the absorption efficiency of the laser pulse, which is a similar result to using a high absorption propellant [58]. Their experiments also found that thicker layers of water produced higher $C_{m}$ values.

\subsection{Modes of Laser Ablation Plume Generation}

There are two methods to generate thrust via laser ablation of the material. Reflective mode(R-mode) is when the laser pulse on the material causes the plume to expand back towards the laser. Transmission mode, or T-mode, involves ablating the thickness of the material with a single pulse so that the plume expands from the back of the material. The following figures describe both reflection (Figure 3-4) and transmission mode (Figure 3-5) ablation. 


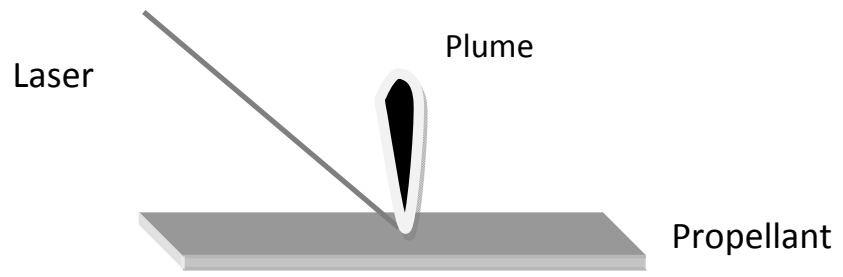

Figure 3-4: Reflective Mode Ablation

The R-mode has several drawbacks, the primary one being that material deposits often end up on the laser lens if the laser is in close proximity to the material. Another obvious drawback is wasted material. As the material is ablated, there is unablated material left over that will not be able to provide the same ablation energy as the previous pulse due to the crater that remains. This material could be made thinner so as to have the laser ablate the thickness in one single pulse and reduce material waste.

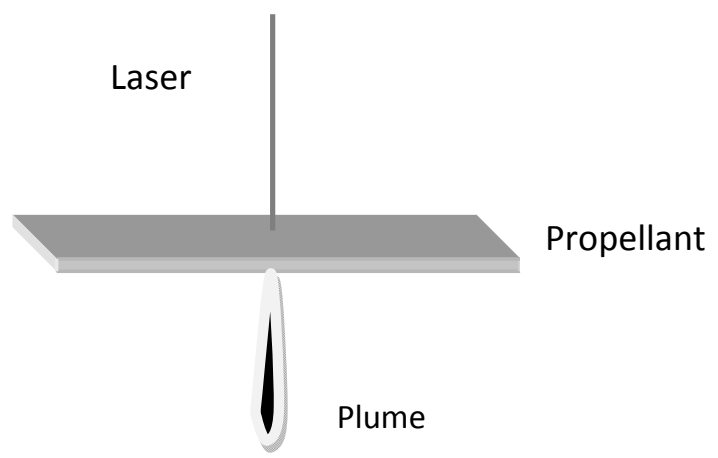

Figure 3-5: Transmission Mode Ablation

The thickness of the material required to completely ablate and achieve transmission mode is dependent on the laser characteristics. Laser power, frequency, and wavelength all contribute to the ability of the laser to ablate. Different materials will also exhibit different performances with different lasers.

For the testing conducted in this research reflective mode was mainly used as the lenses were considerably far away from the ablation zone, and pulse energies are too low to effectively ablate the materials used with a single pulse. Using reflective mode did not hinder any experimental data, and several observations were made based on reflective mode ablation specifically. A test was designed to incorporate transmission mode, however it was unclear as to whether or not transmission mode actually occurred. 


\subsection{Propellants Used for Laser Driven Micropropulsion}

Propellants that are best for laser micropropulsion have several main requirements, one being that they should not continue to burn after the laser has stopped firing. This would mostly waste propellant. The propellant can also not contaminate the body it's propelling with ablation by-products. If contaminants get onto the lenses or sit in a nozzle, they can greatly hinder the performance of the micropropulsion system, if it's able to function any further at all [5].

Polymers are often chosen for applications where thrust is desired. Metals have very high heat conductivity when compared to polymers. For low power lasers, the likelihood for ablation increases when materials with high light absorption in the laser wavelength are chosen.

Diode lasers, which are typically used for micro propulsion, are also usually unable to reach ablation thresholds for metals unless they are used as a pumping source for other lasers, for example a diode

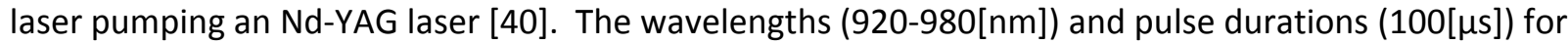
diode lasers limit them to using polymers for ablation. Metals do not eject much mass when ablated, again when compared to polymers for low power lasers, thus it is not very good for thrust production with low power lasers [5]. However, the low thrust production levels of metals and low material removal amounts compared to polymers per laser pulse increase the specific impulse values of most metals.

Polymers generally undergo incubation-the fluence threshold reduces as the material is pulsed with the laser [59]. This means that initially the fluence threshold may be high, but after a number of pulses it will decrease fairly rapidly. Incubation generally occurs at wavelengths where the absorption coefficient of the polymer is low [60].

One investigation showed that the ablation of several different polymers melted and generated volatile substances. These polymers are polycarbonate (PC), polyethylenterephthalate (PET), polytetrafluoroethylene (PTFE), and polymethylmethacrylate (PMMA). Polyimide (PI) was the last test material, which tended to degrade and/or sublimate [59]. All of the materials tested induced cavities smaller than the quasi-Gaussian focal diameter on the surface. The ablation rates were less than $1 \mu \mathrm{m}$ within the first 50 pulses, which was typically due to incubation [59]. 
Ablation of polymers is both a photochemical and photothermal reaction, with the ratio of the two depending on the polymer blend and the irradiation wavelength. For thrust generation, the photochemical properties are not very important [53]. Thrust generation requires exothermic decomposition, gas formation, and a well defined decomposition temperature. Transformation of laser energy to thrust for energetic polymers is typically greater than $100 \%$, implying that $100 \%$ of the energy focused on the material is converted into thrust [53].

\subsection{Doping Agents}

Doping agents are added to materials to assist with absorption of light at wavelengths where the material's absorption coefficient is low. Polymers are generally able to be ablated with less intensity than metals, but doping the polymer compound can increase its ability to produce thrust. Generally no more than approximately $10 \%$ dopant should be added to the material [53]. Most polymers are unable to absorb infra-red radiation unless doped. These dopants could be carbon, or IR dyes. Carbon nanopearls produce the best results from various thrust experiments [53].

Glycidel Azide Polymer (GAP) was originally developed as a binder for solid rocket propellant as it's a high energetic polymer. When GAP is doped with an IR dye it typically transforms almost all of the material ablated to a gaseous form, thus being good for generating thrust. When GAP is doped with carbon, many craters are formed and material is ejected without creating much gaseous product, which is not very good for generating thrust [53]. Generally, IR dyes lead to more homogeneous decomposition, which aids in transforming ablated material to gaseous form and generates more thrust than if other dopants were used. For example, large material fragments are ejected from some materials doped with carbon. PVC $+\mathrm{C}$ (poly vinyl chloride doped with carbon) and PVN $+\mathrm{C}$ (poly vinyl nitrate) are two examples where large material fragments are ejected upon ablation [53].

Lippert \& Urech found that the plume expansion velocity of the explosion from ablation was low for PVC while for GAP and PVN it was fairly high [53]. The amount of chemically stored energy is proportional to the expansion velocity, implying that a high expansion velocity has more energy available for thrust [53]. Eckel et al found that doped materials tested had much higher exhaust velocities than the undoped materials; $2.5 \mathrm{~km} / \mathrm{s}$ vs. $8 \mathrm{~km} / \mathrm{s}$ when doped [55].

\subsection{Basic Principles of Thrust Production}

For thrust production, a high transition rate from solid state to gaseous state is desired [53]. Ablation is caused by radiating a large amount of energy into a spot on the material in a very short period of time. 
The energized material then transitions from solid to gaseous form and exerts an impulse on the body [5]. The ejection of gas from the surface forms a cloud of plasma particles, which consists of ground state neutrons, electrons, and ions [49]. The mass and exhaust velocity of the ablated material are the main contributors to the production of thrust. Mass, as defined in these equations, is the amount of material ablated per pulse. Below in Equation (3.10) is a commonly used rocket thrust equation [6].

$$
T=\dot{m} V_{e x}
$$

Where $\dot{m}$ is the mass flow rate of the propellant, and $V_{e x}$ is the effective exhaust velocity of the propellant. The effective exhaust velocity is commonly used to describe a uniform, axial, and averaged exhaust velocity of the ejected propellant. Since multiple pulse ablation is used for thrust generation in this work, the approximated average velocity of a single pulse is used for calculation. The product of the mass flow rate and velocity is related to Newton's second law, shown in Equation (3.11).

$$
\begin{aligned}
T & =\frac{d}{d t}\left(m V_{e x}\right) \\
& =\frac{d m}{d t} V_{e x}+m \frac{d V_{e x}}{d t} \\
& =\dot{m} V_{e x}
\end{aligned}
$$

Since exhaust velocity is considered constant, the change in velocity over time is zero. The change in mass is the mass removed from the sample via laser ablation, hence mass flow rate, $\dot{m}$. In this equation, $T$ is the thrust generated, and $V_{e x}$ is the effective exhaust velocity of the ablated material.

By integrating force, or thrust, over the time interval the force is applied, one can find the Impulse of the action, described by Equation (3.12).

$$
\begin{aligned}
I & =\int_{t i}^{t f} T d t \\
& =\int_{t i}^{t f} \frac{d m}{d t} V_{e x} d t \\
& =\Delta\left(m V_{e x}\right)
\end{aligned}
$$

This shows how Impulse, $I$, is defined as the change in momentum. The product of mass and velocity is known as momentum, P, typically given in Ns. The relation is described below in Equation (3.13). 


$$
\begin{aligned}
I & =\Delta\left(m V_{e x}\right) \\
& =\Delta P
\end{aligned}
$$

Where $\mathrm{m}$ is the mass ablated per pulse and $V_{e x}$ is the effective exhaust velocity over the time interval used to measure thrust. The use of impulse to calculate exhaust velocity is used in these experiments with the assumption that the initial velocity is zero as the propellant starts from rest.

The moment coupling coefficient is often used to evaluate micropropulsion systems, described with Equation (3.14) as the thrust to power ratio [61].

$$
\begin{aligned}
C_{m} & =\frac{P}{E} \\
& =\frac{m V_{e x}}{E}
\end{aligned}
$$

Moment coupling coefficient, $C_{m}$, often in units of $\mu \mathrm{Ns} / \mathrm{J}$ or $\mu \mathrm{N} / \mathrm{W}$, is a very useful term when comparing data from one test to another as it takes the energy in each pulse into consideration. The term however does not account for the many other parameters that influence thrust output from laser ablation such as pulse width.

By using the moment coupling coefficient, thrust can also be calculated using Equation (3.15) [5].

$$
T=C_{m} v E
$$

Where $v$ is the frequency of the laser and $\mathrm{E}$ is the laser pulse energy. This equation suggests that thrust is directly proportional to the repetition rate of the laser. However, use of this equation also suggests that the force on the body is present for the duration of time defined by the repetition rate. This method was used for initial calculations and determined to be incorrect for the system used in these tests. Thrust generated for a time of $1.5 \mu \mathrm{s}$ was used to calculate impulse in these tests as suggested by Pakhomov et al.[18]. Thrust is often determined experimentally, and then other parameters are calculated via these equations by using the ablated mass, thrust generated, and time interval.

Every material will react differently with different wavelength lasers. This will ultimately determine the amount of energy that the material absorbs from the laser, and thus determines the thrust potential for the material [3]. The amount of energy required to ablate a material can be calculated using Equation (3.16) [4]. 


$$
Q^{*}=\frac{E}{\Delta m}
$$

Where $\mathrm{E}$ denotes the amount of optical power per pulse provided by the laser in Joules, and $\Delta m$ is the mass of the material ablated. Using this value with the coefficient of momentum $C_{m}$ from Equation (3.14), Equation (3.17) can be formulated for exhaust velocity [62].

$$
V_{e x}=C_{m} Q^{*}
$$

With high powered lasers, metals can prove very effective for thrust production as they are very easily capable of surpassing fluence thresholds. Metals also typically have high exhaust velocities, which would directly affect thrust produced [5]. Specific impulse as defined in Equation (3.18) as the ratio of thrust per unit weight of material used and is very useful when comparing propulsion systems.

$$
\begin{aligned}
I_{s p} & =\frac{1}{W} \int_{t_{0}}^{t_{f}} F d t \\
& =\frac{1}{W} \int_{t_{0}}^{t_{f}} \frac{d P(t)}{d t} d t \\
& =\frac{P}{W} \\
& =\frac{V_{e x}}{g_{0}}
\end{aligned}
$$

Weight, W, in Equation (3.18) is defined as the product of mass and gravity. The mass term drops out and another equation to define specific impulse remains as the time averaged exhaust velocity divided by gravity at sea level. A term used to describe the ablation efficiency is given in Equation (3.19) [5].

$$
\eta_{a b}=\frac{m V_{e x}^{2}}{2 E}
$$

Where $\eta_{a b}$ is the ablation efficiency and $\mathrm{E}$ is the laser pulse energy. The laser ablation efficiency is simply the kinetic energy of the exhaust divided by the laser pulse energy. It can also be described with Equation (3.20) where an arrangement of terms shown can provide another relation [4]. 


$$
\begin{aligned}
\eta_{a b} & =\frac{m V_{e x}{ }^{2}}{2 E} \\
& =\frac{C_{m}{ }^{2} Q^{*}}{2} \\
& =\frac{C_{m} V_{e x}}{2}
\end{aligned}
$$

This shows that the relation to the ablation efficiency is directly related to the exhaust velocity and the moment coupling coefficient. It's interesting to note that for very high values of $C_{m}$ it is likely that the exhaust velocity, and in turn the specific impulse value, will be low due to the ablation efficiency threshold of $100 \%$. 


\section{Chapter 4: Test Apparatus Development}

Ready apparatus' for testing micro thrust are non-existent, and any method for testing must be designed at the facility. As a result, test apparatus development was required early in this thesis before any testing had ever been conducted. Also, as such testing has never been conducted at these facilities it was required to fully investigate and develop all methods from scratch.

\subsection{Testing Method Development}

The apparatus had several different initial concepts that were derived from published methods. The original methods and final setup are discussed in this section.

\subsubsection{Torque Sensor}

The initial design method was to have a rod attached to a torque sensor, pivoting on bearings. On the rod would be a sample holder to which the laser would be directed for ablation. The ablation would cause a deflection, and in turn torsion of the rod. The holder was designed to be adjustable to create a larger moment arm. The design is described below in Figure 4-1.

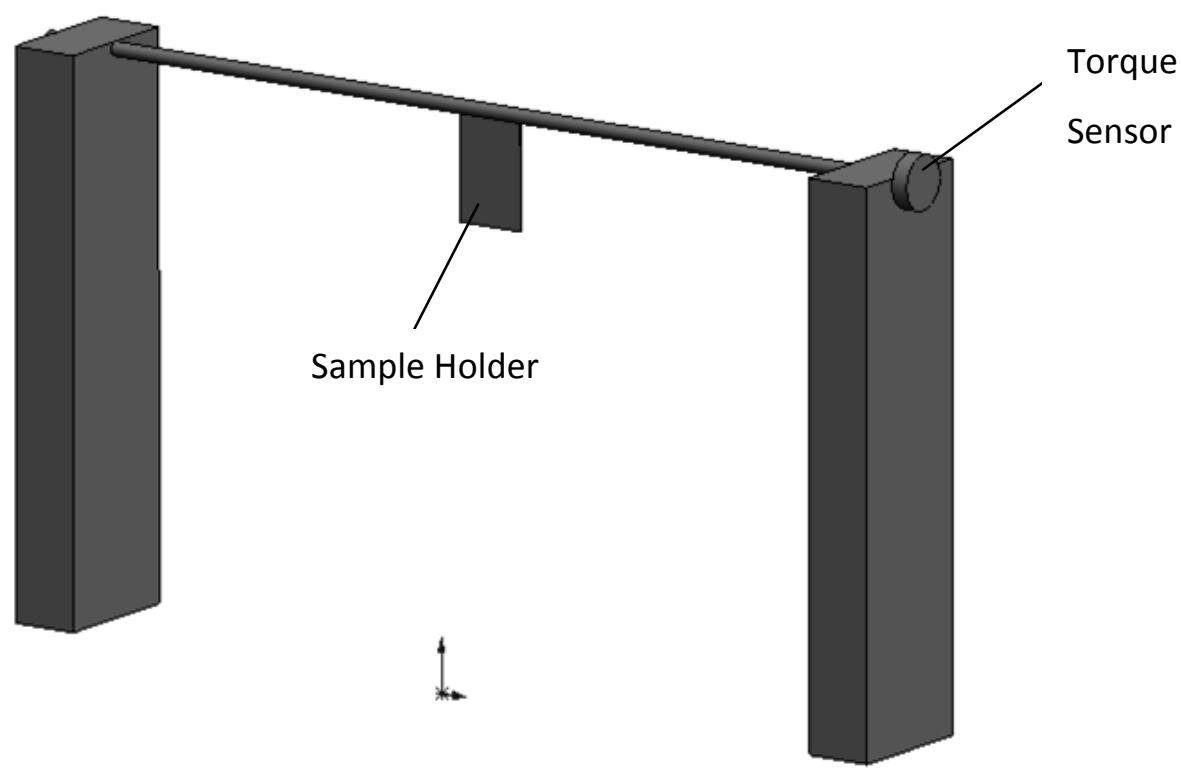

Figure 4-1: Torque Sensor Design

The issue with this design is the torque sensor sensitivity, and adequate bearings that would not essentially drown the micro forces from the ablation. The sensor would also require a series of components (amplifier, controller etc) that were too costly to purchase without a guarantee that it 
would work. A vacuum chamber is believed to aid in the acquisition of micro thrust data and was originally part of this design, similar to published methods. As a vacuum chamber was not available, on top of the other complications, this design was discarded.

\subsubsection{High Speed Camera}

A high speed ICCD camera was also investigated in order to capture exhaust velocities and solve for the thrust developed. As time of flight (TOF) measurements do not work well in atmospheric pressure since ambient pressure restricts plume expansion [35], and the equipment is very costly, this method was quickly discarded.

Plume expansion restriction is also the reason that another method was discarded, where the ejected material would push on a pendulum.

\subsubsection{Direct Force Sensor}

This method would employ a direct force sensor: PCB Piezotronics model 209C01, capable of $100 \mathrm{kHz}$ resolution. It was originally believed that $100 \mathrm{kHz}$ was too slow which was an initial downside to this method. This sensor is also very costly itself, in addition to the cost of the amplifier and the controller. The sensor is believed to be accurate and has been used by Pakhomov et al. in several instances, however a proper setup to utilize this sensor without damaging it was a concern, as testing for both reflective and transmission mode was desired. It was also desired to look into other methods for higher resolution, so this method was discarded.

\subsubsection{Piezoelectric Sensor}

After finding the direct force sensor from PCB Piezotronics (Depew,New York), and deciding that a direct force measurement system would be best for these facilities, another method for testing using piezoelectric materials was designed. This method used piezoelectric films from Measurement Specialties, Inc. (MSI) made of polyvinylidene fluoride (PVDF). When a force is applied to the film, the film displaces in one or more of its axes and outputs a voltage relative to the displacement. The sensors are very sensitive and, if used in the proper mode, have a very high resolution in the $\mathrm{MHz}$ range. As the films are very susceptible to being burned by the laser, a method of force transfer was designed. Initially, the system was set up to have a pendulum hold the sample, and have the pendulum impact the sensor. The sensor has a very high resolution when plane 1-2 is laid flat against a surface and is impacted along Axis 3, described in Figure 4-2 where a film and its axes are shown. Axis 1 is the axis on which the attached electrical leads are parallel to. 


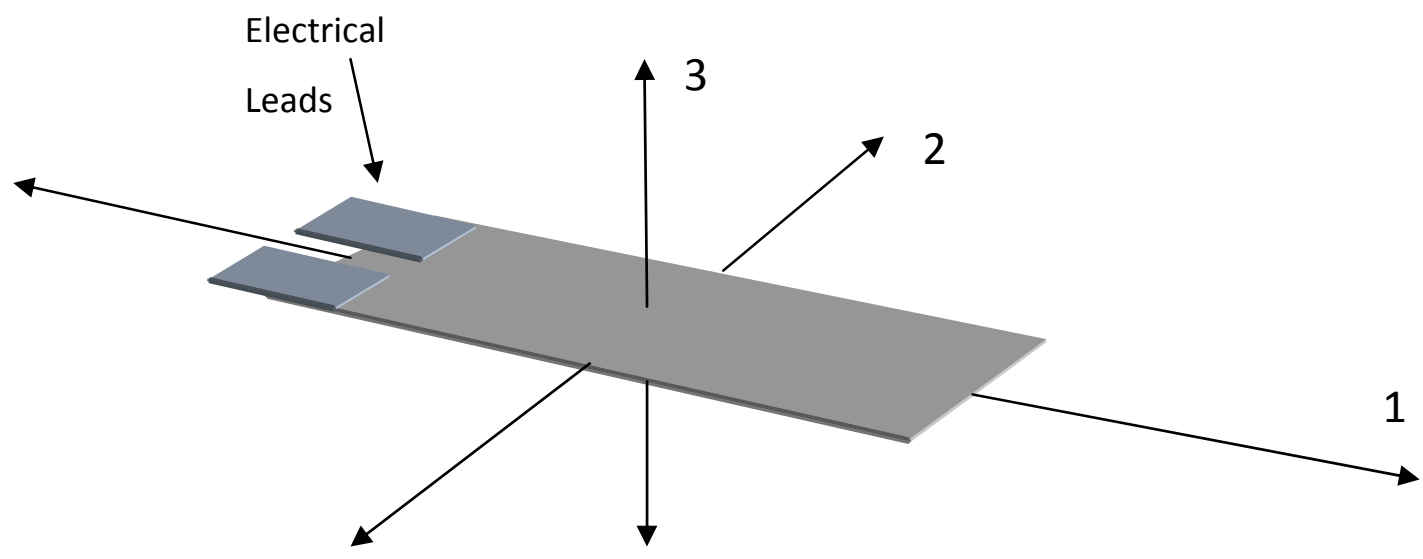

Figure 4-2: Piezo film axes

The manufactured apparatus design is shown below in Figure 4-3.

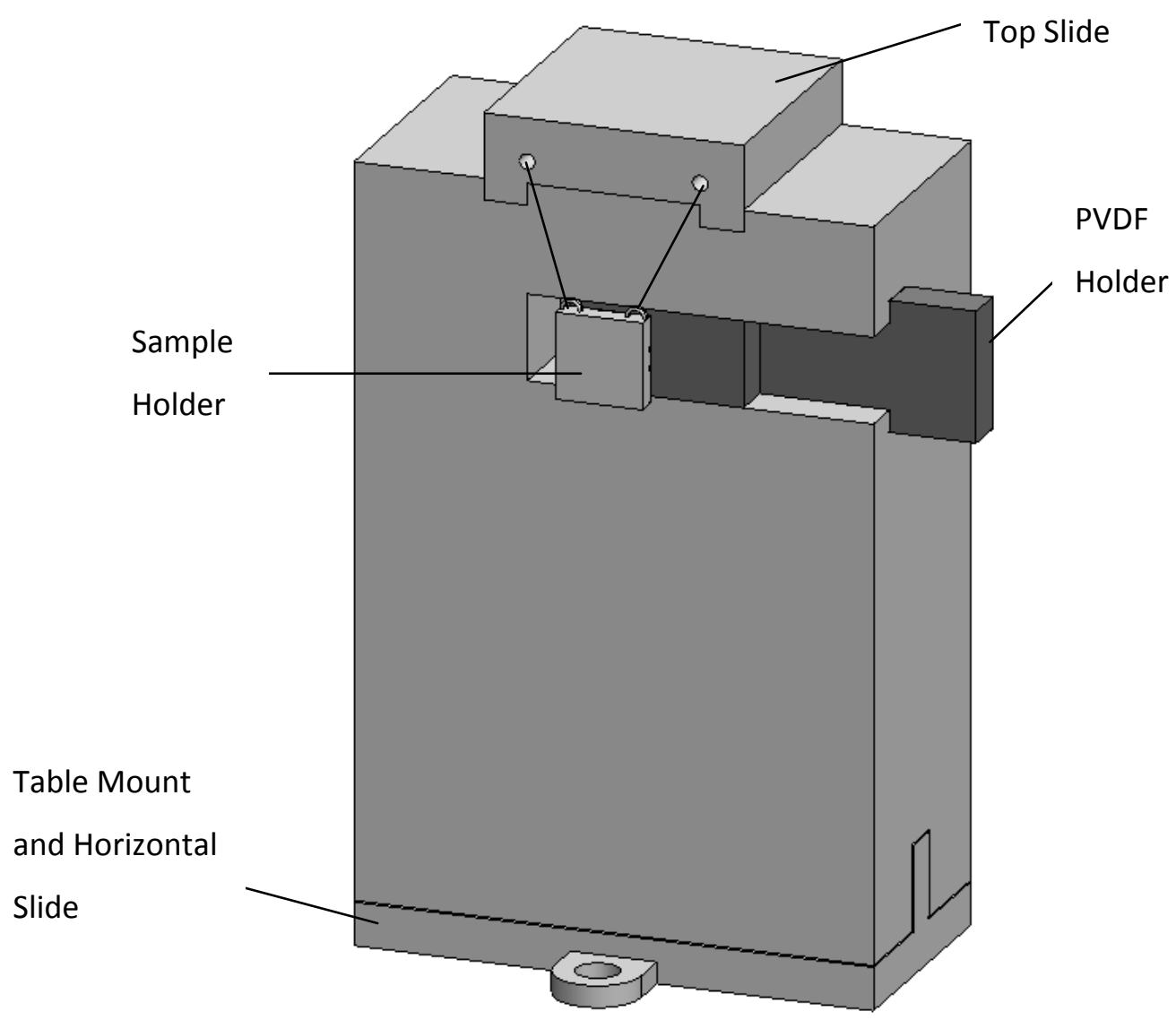

Figure 4-3: Piezoelectric Test Stand 
The design was kept simple so that it could be manufactured by the writer with the facilities at the university. The propellant would be attached with an adhesive to the visible face of the sample holder, which was to be suspended by thin wire from the top slide where vertical adjustments of the sample holder can be made. The table mount could be attached to the table, and served as a horizontal adjustment method for the sample. The horizontal and vertical adjustment methods were added since the laser level and placement is set for these tests.

The sample holder conically comes to a point on the opposite side, and is in contact with the PVDF on the holder. The PVDF holder, as shown in Figure 4-4, slides out of the stand in order to change the sensor or insert a different slide with a different profile, described below.

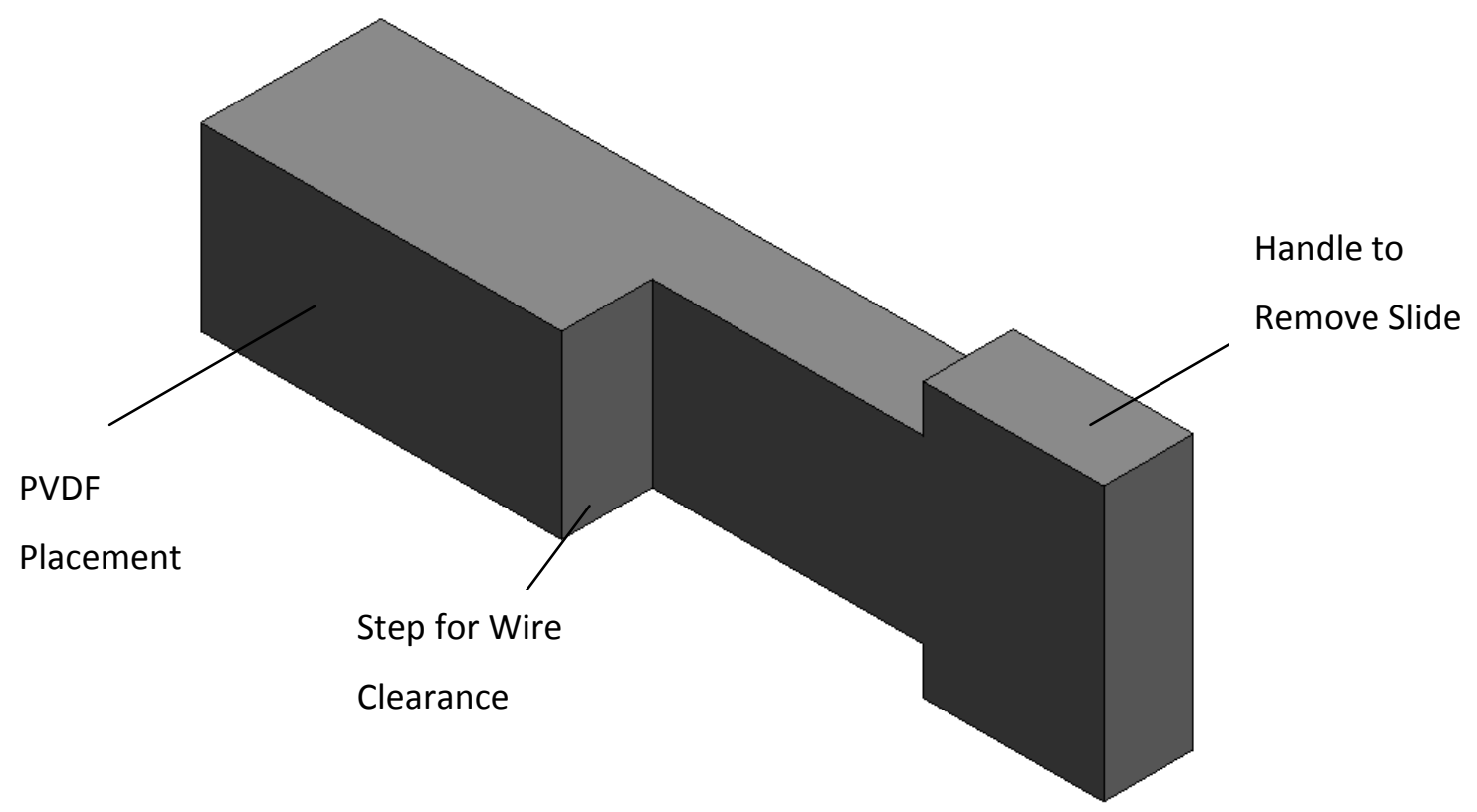

Figure 4-4: PVDF Slide 1

The step in the center of PVDF Slide 1 allows for wires to be run without bending the film and putting stress on the adhesion. The PVDF was connected via a twisted shielded cable to an amplifier that was designed for these tests, described later in this chapter. This system was believed to have been able to work, except the forces are so low that the sensor is unable to pick up the signal when applied in this fashion. The voltage to force conversion when the force is applied for film compression on the 1-2 plane is very low, approximately 13E-3V/N [63]. The PVDF film is much more sensitive in its other modes; however the response is much slower. The tradeoff between response and sensitivity was easy to debate since the signal was regularly non-existent in the compression mode. The slide was redesigned to allow for bending in the film, shown below in Figure 4-5. 


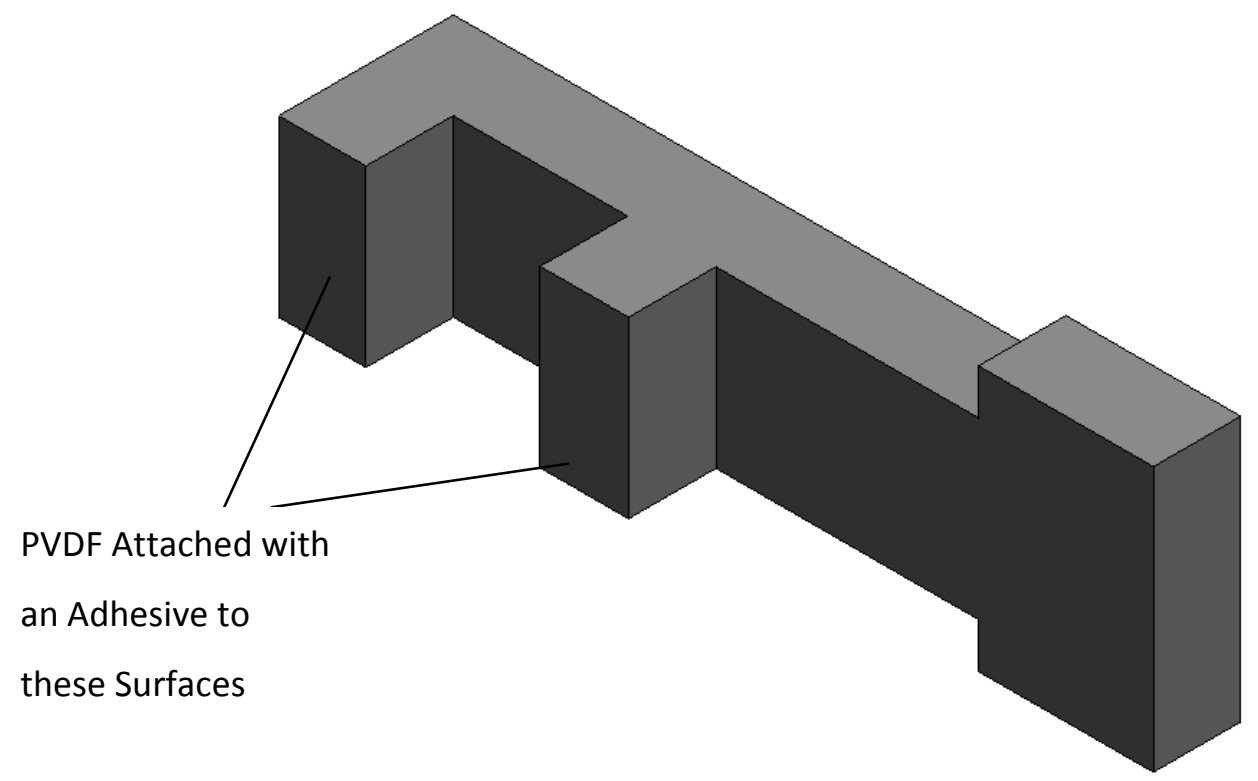

Figure 4-5: PVDF Slide 2

Bending was a relatively simple method to choose from the initial as it required very little machining of the test apparatus. The samples were tested again and results were recorded. The issue with this method is that the data is inconsistent, which lead to the assumption that the sample holder is also providing error to the data. The sample holder is a mass that the force must provide momentum to in order to then displace the PVDF film. Since this is not a perfect system there are losses with each transfer of momentum, also with contributions to the minimal but existent air resistance. The other potential issue is that the sample holder twists when a force is applied, implying that the force is not being directly transferred to the PVDF film as intended.

Due to the complications with this design, another final design was tested using the same concept of directly sampling the force using a PVDF film. This new method is described in the next section.

\subsubsection{Cantilevered Piezoelectric Sensor}

Building on the bending mode of the PVDF film, a new method was developed for testing thrust production via laser ablation. This method removes the sample holder and allows the laser to ablate a propellant which is directly attached to the sensor. The test apparatus is designed to exclude the sensor from the ablation area itself in order to minimize any contribution from the ejected material on the sensor directly. 
This setup provided a well distinguishable signal and was further developed to reduce noise, and isolate the force produced. Data acquisition is achieved by shooting the laser at the propellant attached to the sensor, as shown in Figure 4-6.

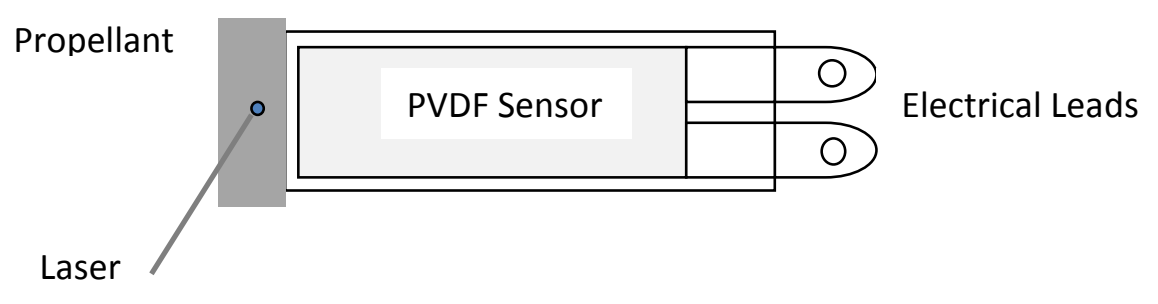

Figure 4-6: Propellant Attached to PVDF Sensor

The propellant is attached using Loctite 495 as it was found to keep the PVDF film lamination undamaged upon propellant removal. An example of lamination damage is shown below in Figure 4-7.

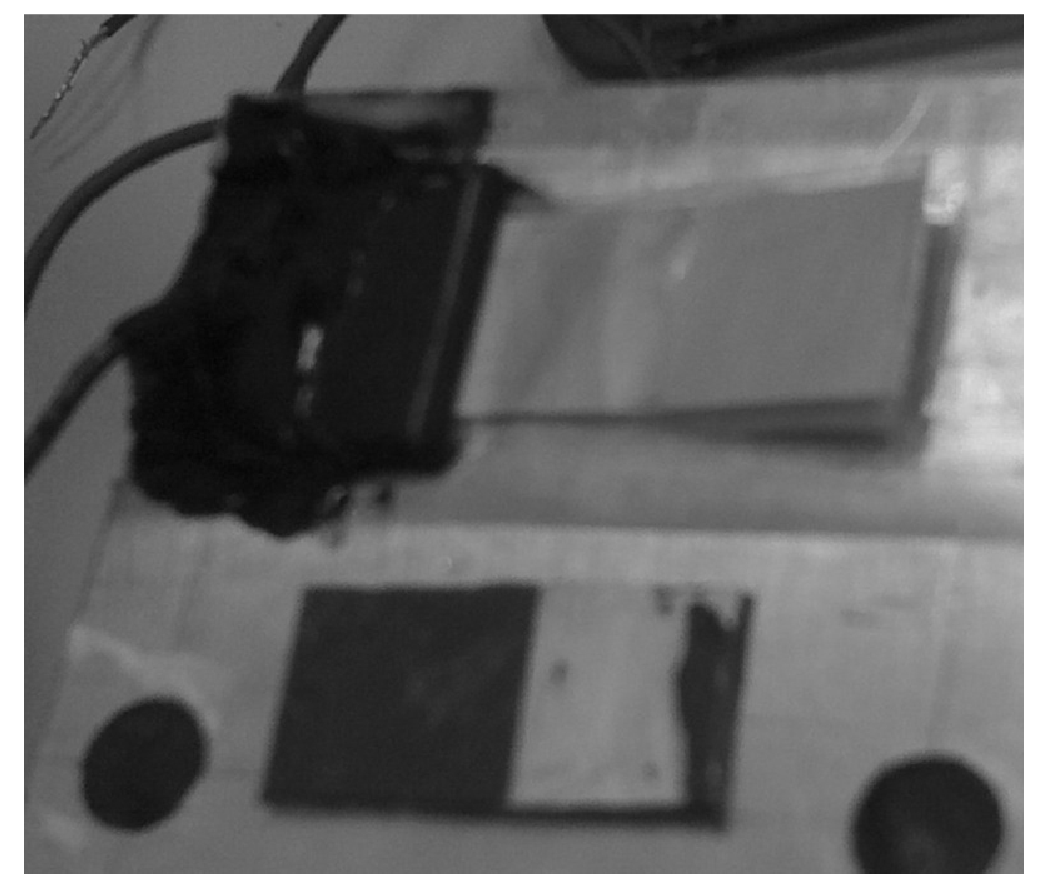

Figure 4-7: Lamination Damage

The bottom rectangular object is a sample that was glued to the PVDF film. A silver substance can be seen on the sample, which is PVDF that went with the sample when they were separated. The Loctite 495 seemed to reduce the damage to the sensor and was recommended by MSI. In order to further protect the sensor, the propellant is attached at the tip of the PVDF film on the lamination instead of on 
the PVDF directly; as was described in Figure 4-6. This also protects the sensor from the laser beam once the material has depleted at the laser spot. PVDF films were also initially chosen due to their very low cost, and in the event that a sensor was damaged it could easily be replaced. Using this method very few sensors were used and virtually none were damaged unintentionally. Several of the sensors were damaged intentionally by the beam to observe the effects of the resulting sensitivity. It was observed that the sensor generally was unusable once the laser ablated the surface. This is not necessarily due to the fact that it was punctured but could be due to the overheating of the element, or the fusion of the two layers shorting the sensor. The sensors can accept temperatures in the range of $40^{\circ} \mathrm{C}$ to approximately $100^{\circ} \mathrm{C}$ [63], so the very high temperatures encountered from a direct ablation would definitely destroy the sensor. Often once the sensor is ablated it was observed to no longer output a signal at all.

This setup illustrated in Figure 4-8, allowed the PVDF to deflect freely from its base. The mounting holds the sensor steady as to not induce an erroneous signal, and the wires connected to the leads are twisted and shielded with an aluminum foil to protect from electromagnetic interference (EMI). The aluminum foil is grounded to provide further protection from EMI.

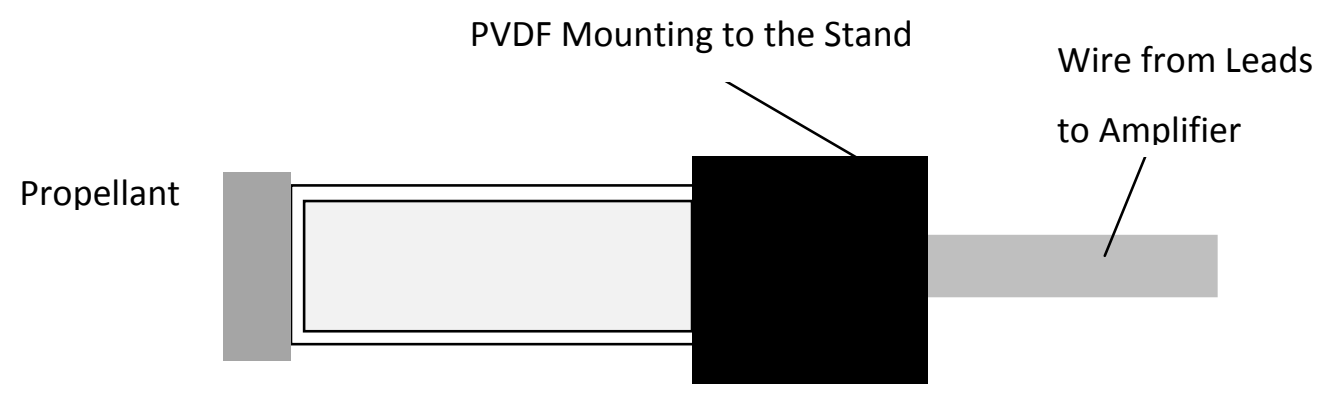

Figure 4-8: Mounting for PVDF Sensor

The force applied at the ablation site deflects the PVDF by $\delta \mathrm{d}$ when the propellant is ablated, described in Figure 4-9, which then outputs a signal proportional to the deflection as a potential difference. 


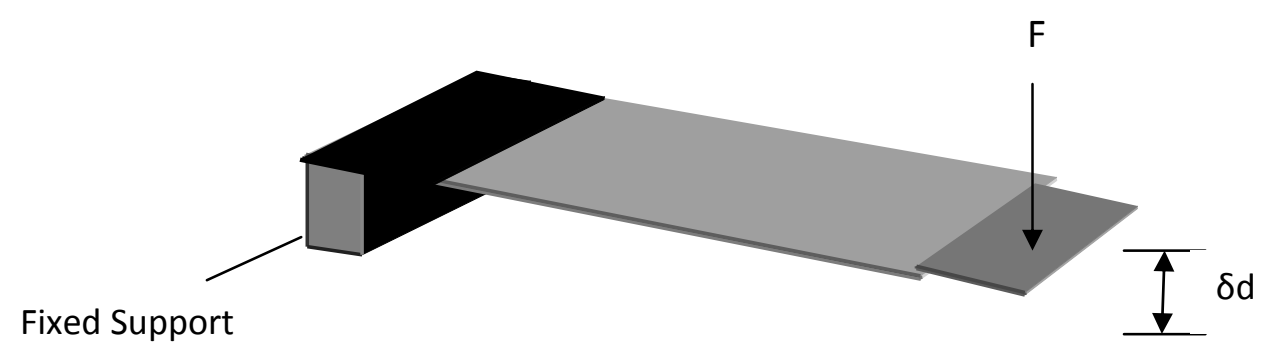

Figure 4-9: Applying a Force Along the 3 Axis

The leads attached to the PVDF film are both attached to a different layer on the film where the potential is generated. For bending, both layers are off of the neutral axis. As the sensor bends, the neutral axis acts as a pivot for the layers and enhances the compression of the layers as a result; described in Figure 4-10 below.

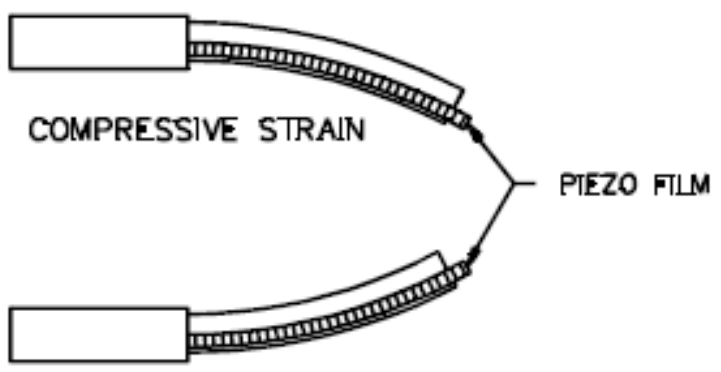

TENSILE STRAIN

Figure 4-10: PVDF in Bending [63]

The actual sensor setup is shown below in Figure 4-11, where the sensor is attached to a 3-axis with fine adjustment for laser focusing. The platform is securely fastened to the table to reduce error during testing from accidental movement during adjustment. 


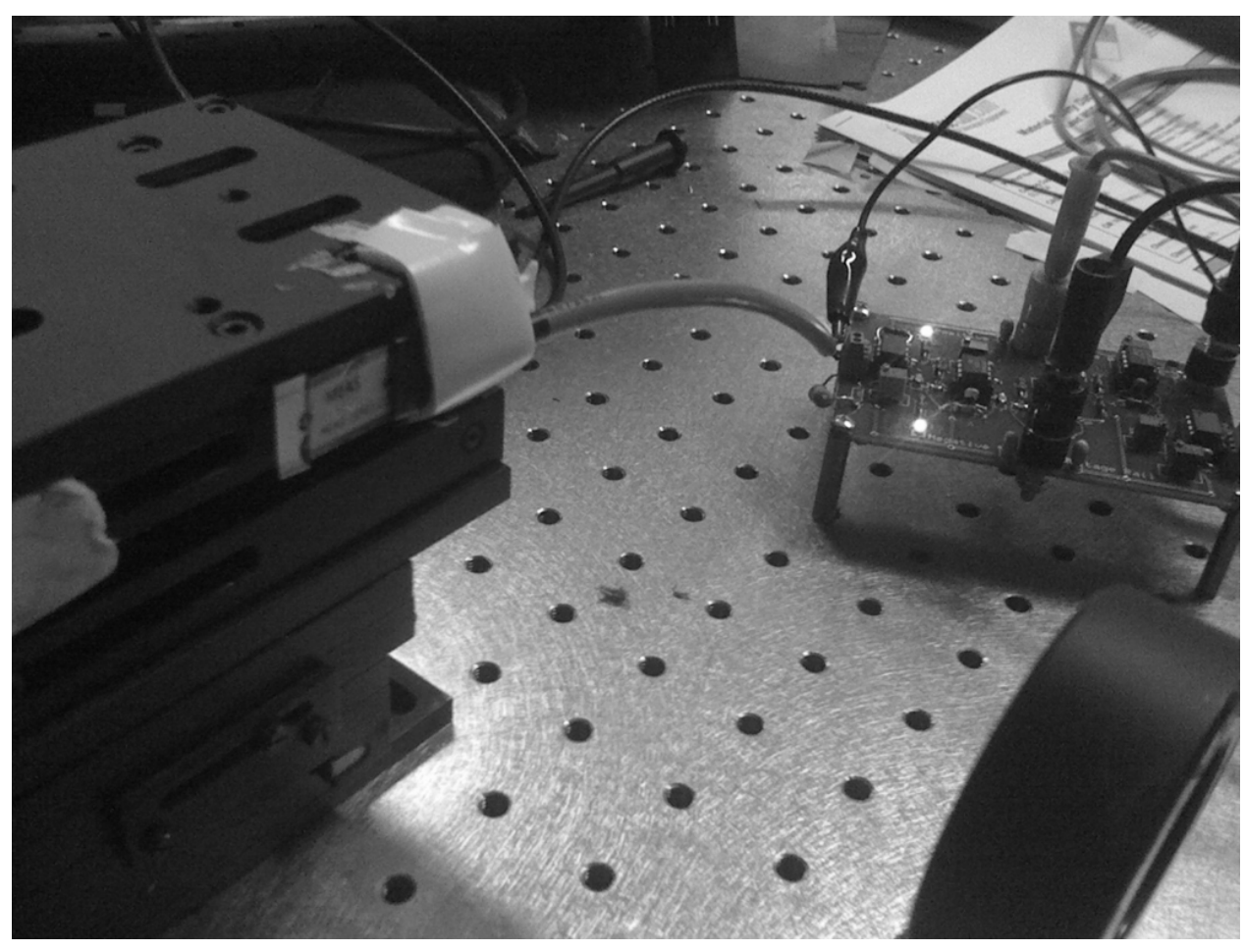

Figure 4-11: Sensor on Platform

Figure 4-12 below shows the laser ablating the propellant during an actual test.

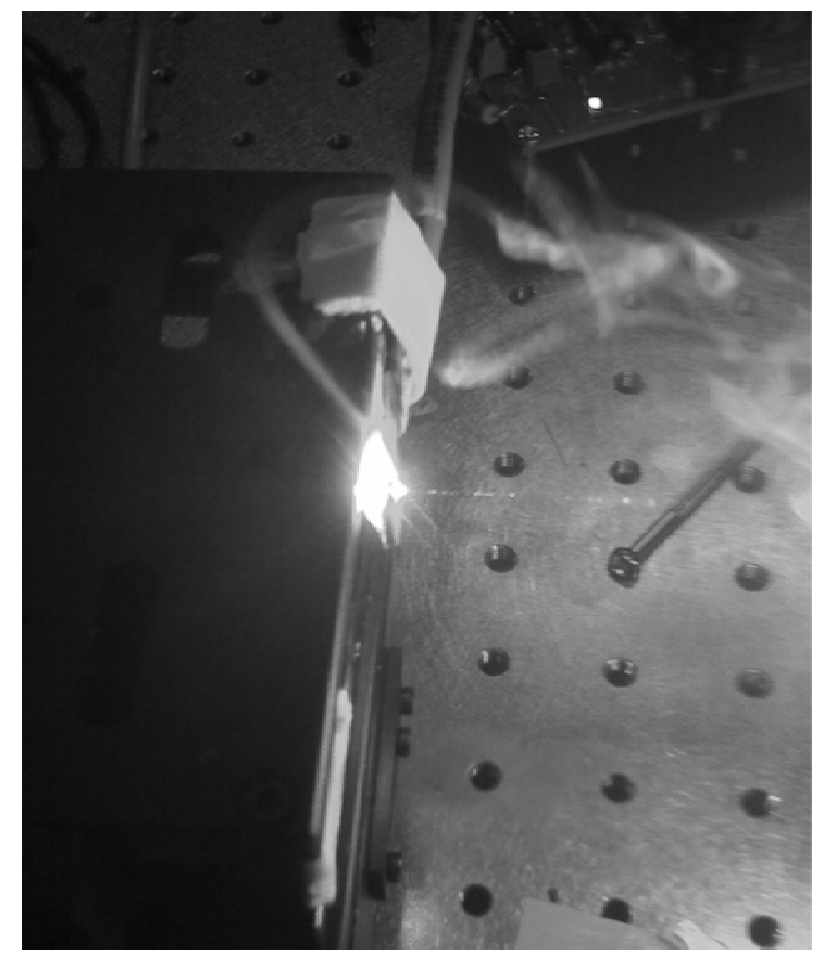

Figure 4-12: Laser Ablating Propellant 


\subsubsection{PVDF Film Sensor Issues}

There is no data available for the response of the PVDF film in a cantilevered arrangement. This became an issue as calibrating for such small interrupted forces, as those generated from a highly repetitive pulsed laser, is very difficult. Calibration methods are explained later in this chapter under Calibration. Due to the calibration difficulties, the option to attach the film to a thin surface and attempt to have the thrust stretch the PVDF in the 1 axis arose. This method would be satisfactory for larger forces, but the thrust generated is believed to be too small to be able to deflect such a surface. The MSI technical manual [63] mentions that the sensitivity of the sensor is heightened when used in bending, however to an unknown degree. There is data available for the compression and tension modes, which is why those modes were experimented with initially.

Shooting the propellant at different positions is assumed to provide different results due to the operation of the PVDF responding to a displacement with a voltage. As a result, the effect of the distance to the voltage change will be investigated as well. For these experiments, all samples were ablated at the same distance from the PVDF sensor while keeping a safe distance to minimize pyroelectric effects. The pyro-electric effects are low frequency [63] and are assumed to be filtered out by the amplifier and post process filtering. Typically only a very rapid change in temperature would be noticed [63], however, any pyro-electric effects are still best avoided during testing.

\subsection{Piezoelectric Sensors}

The pyroelectric phenomenon was discovered by Gaugain in 1856. He made precise measurements of the pyroelectric charges using a self discharging electroscope. In 1878 Lord Kelvin then associated the electric field with the pyroelectric effect [64]. Piezoelectricity, translates from the Greek language as pressure electricity [65][66], was then discovered by the Curie brothers in 1880 [67]. They noticed electric polarization occurred when a crystal was compressed along its hemihedral axis [67]. The crystal produces equal and opposite electrical quantities, and the quantity developed by a temperature or pressure increase is equal and opposite to that for an equal decrease in pressure or temperature. This implies that the piezoelectric and pyroelectric responses are completely reversible [66]. The electric charge, however, decays with time so these sensors are not good for static measurement [65].

Piezoelectricity provides great capability for small force sensors. The difficulty with using piezoelectric materials for sensors is proper calibration and filtered amplification. For these experiments, Mylar laminated PVDF (polyvinylidene fluoride) films (LDTK0-028K) were used instead of ceramics due to their 
higher sensitivity. The LDTK0-028K has a thickness of $28 \mu \mathrm{m}$ un-laminated, with a $127 \mu \mathrm{m}$ Mylar laminate.

PVDF piezoelectric films are able to output 10 times the voltage of piezo ceramics with the same force applied. The PVDF films also have a wide frequency range of $0.001 \mathrm{~Hz}$ to $10^{9} \mathrm{~Hz}$, making them highly susceptible to pressure waves and any thermal changes on the film surface [63]. Good shielding is required for use in these applications as the leads are highly susceptible to electromagnetic interference. For these tests, the wires attached to the leads are twisted to dampen major incoming differential signals due to EMI. The wires are then wrapped in aluminum foil and sheathed with a rubber sleeve. At the leads, the wires are quickly spot soldered as to not overheat the sensor but to allow the wires a clean connection. The leads are then taped and wrapped with aluminum as well to reduce any EMI. Using these sheathing methods and proper grounding significantly reduced the amount of noise in the signal.

For mechanical to electrical conversion using the PVDF film, values of $14.4 \mathrm{~V} / \mathrm{N}$ applied can be output at the leads if used in axial tension axis (along axis 1) [63]. Adhesion of the film to a substrate is required, and attempting to deflect the substrate with the expected micro Newton forces was expected to provide very poor results. As a comparison, a complete 3 direction compression would only output 13E$3 \mathrm{~V} / \mathrm{N}$ of force applied [63]. Tests were initially conducted with compressive forces on our apparatus and were very difficult to distinguish if visible on the oscilloscope at all. As was described in the previous section, another method of testing was developed where the film acted in a cantilever configuration. The difficulty with this configuration is that there is very little data available for the PVDF in a cantilevered configuration. Typically this configuration is used for lower frequency applications such as counting, or switches. Early in the experiments, we were unsure as to which method of data analysis should be used. It was decided to experiment with the cantilevered configuration and assume that the thrust generated is low enough to allow the PVDF film to sense a majority of the pulses with our high repetition rate laser. A SolidWorks simulation was performed to estimate the deflection of the film with a $100 \mu \mathrm{N}$ force applied at the center of the sample; Figure 4-13. 


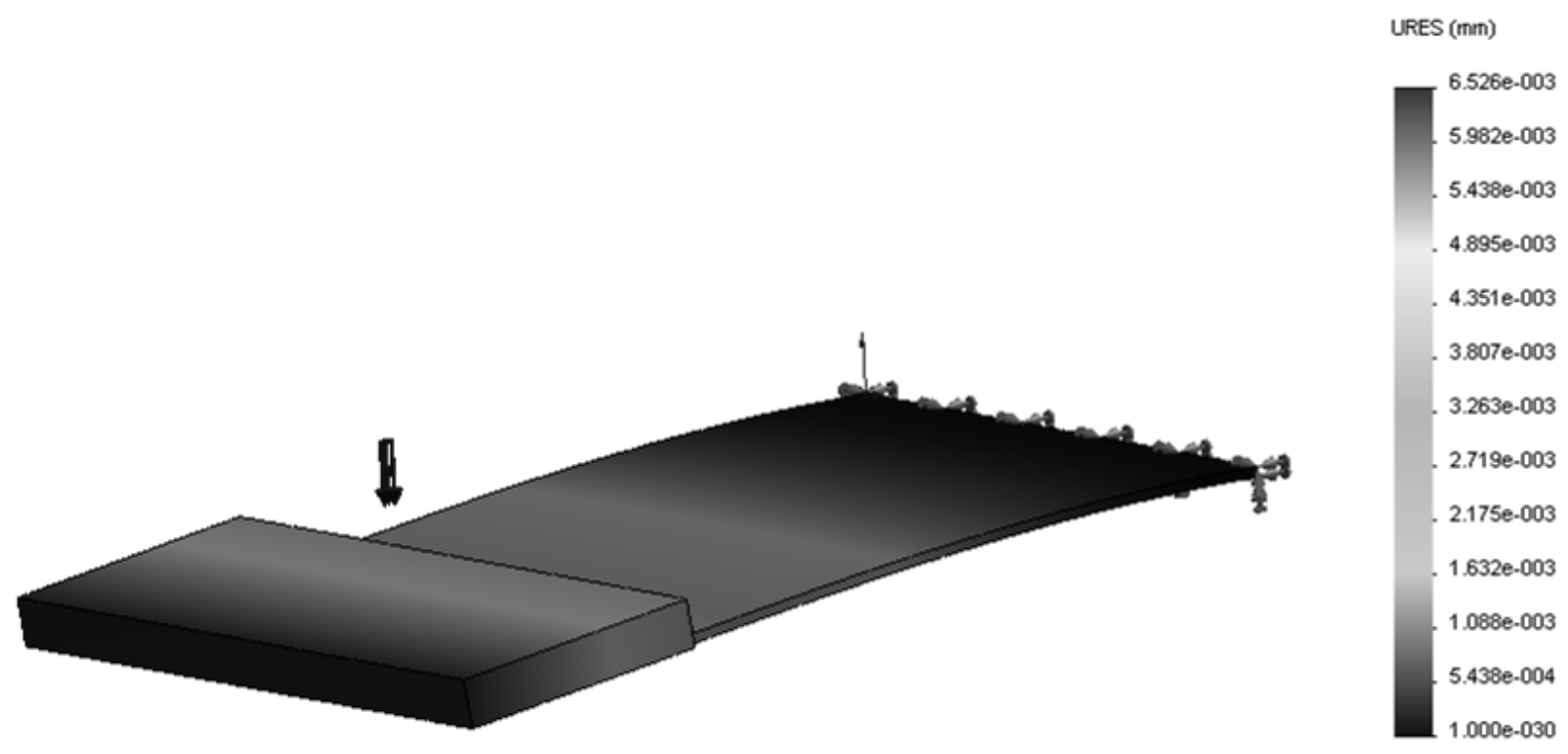

Figure 4-13: Deflected PVDF Film

The film's displacement from a $100 \mu \mathrm{N}$ force is approximately $6.5 \mu \mathrm{m}$, implying that the film will not have displaced a great amount before the next pulse is ablating the propellant. This is also coupled with the natural dampening of the sensor, leading us to assume that the addition of displacement from each sequential pulse is relatively null. As this thesis was not focused on FEM analysis of the sensor during the ablation process, it should be noted that the simulation generated for this section is solely for assumption verification.

\subsection{Signal Processing}

Piezoelectric films are very susceptible to noise, and require extensive filtering and signal processing. For these experiments, the analog signals received from the piezoelectric film are amplified and sent to a digital oscilloscope as an analog signal. The signal is then placed into a developed MATLAB code that converted and filtered the signals using Simulink. MATLAB code and Simulink filter setup are given in Appendix A: MATLAB Code and Simulink Filters.

\subsubsection{Amplifier Development}

The thrust produced by laser ablation is miniscule compared to the minimum threshold for traditional static or dynamic force sensors. The piezoelectric film required amplification to be able to measure the thrust generated. As the signal is also very noisy, an amplifier and filter were required to be developed. Several different options were reviewed and some were assembled onto a breadboard for signal testing. 
One circuit design by Vargas et al. claimed to be able to drive a PVDF film up to $500 \mathrm{kHz}$ [68]. The circuit was assembled onto a breadboard, as shown in Figure 4-14. The circuit did not seem to perform as intended, so this amplifier configuration was discarded.

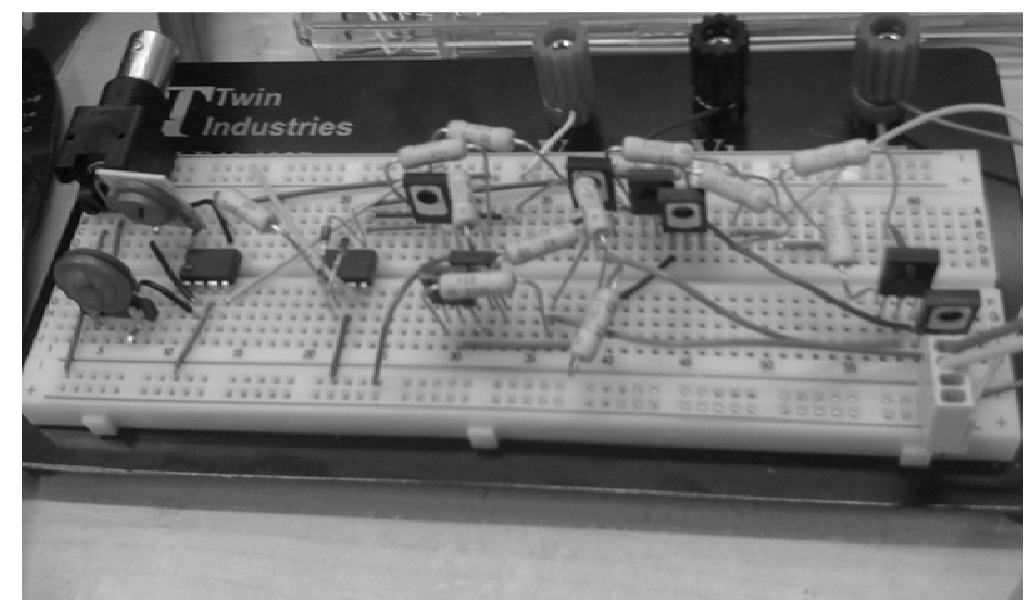

Figure 4-14: Vargas et al. Circuit on a Breadboard

Another amplifier arrangement was developed using Filter Pro 2.0, freely available from Texas Instruments. The developed signal conditioning circuit was converted to a schematic and the board sent out for manufacturing. The electronics were then assembled onto the PCB pictured in Figure 4-15. The circuit schematic is in Appendix B: Amplifier Schematics.

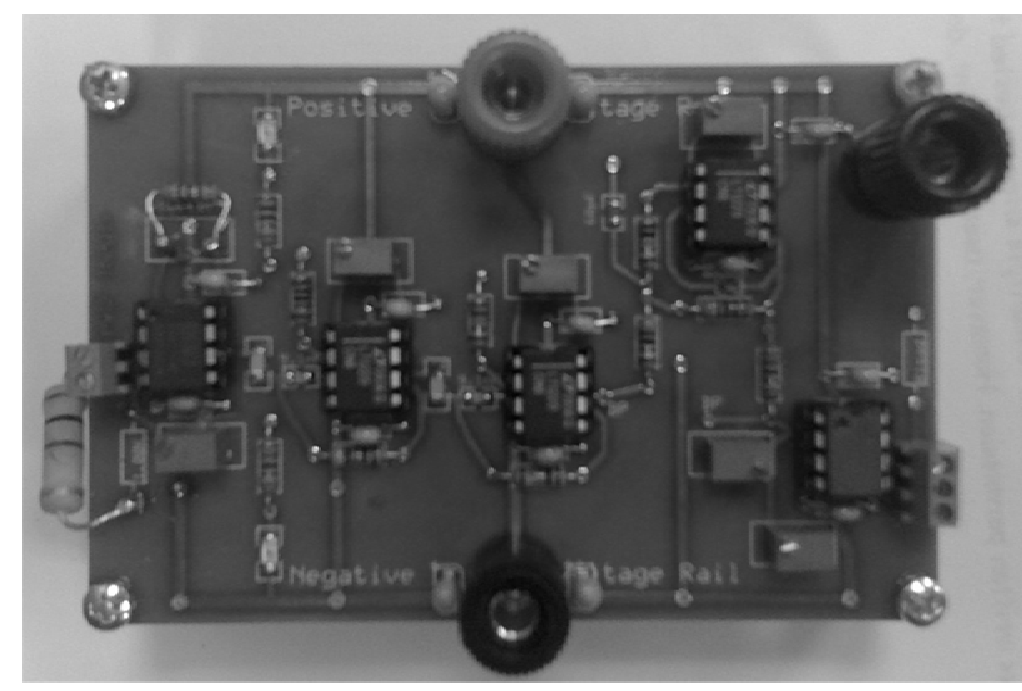

Figure 4-15: Signal Conditioning Unit

The signal conditioning unit was designed to pre-amplify the signal, filter it, and then amplify it again before the signal was sent to a digital oscilloscope. The total gain of the amplifier circuit was 1000 or 
$60 \mathrm{~dB}$. The filter that was used was a Butterworth bandpass type with cutoff frequencies of $1 \mathrm{kHz}$ and $600 \mathrm{kHz}$. The Butterworth type was chosen as its response is flat in the passband. The pre-amplifier stage used an instrumentation operational amplifier due to its high input impedance and easy gain selection through a single resistor. The signal conditioner was tuned and the performance verified using a signal generator [Tektronix AFG 3022B] and oscilloscope [LeCroy Wavesurfer 422] over the frequency range of $1 \mathrm{~Hz}$ to $1 \mathrm{MHz}$; amplifier frequency response graph shown in Figure 4-16.

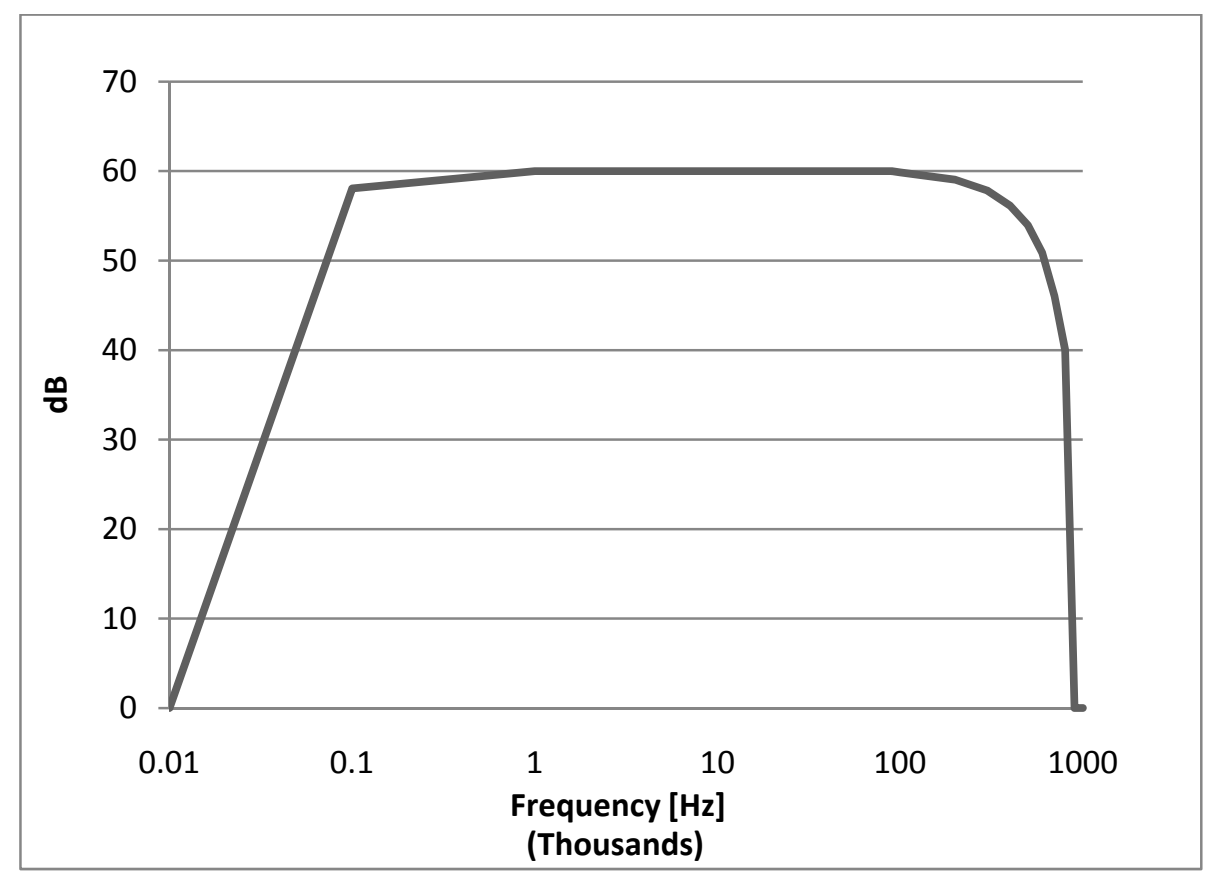

Figure 4-16: Amplifier Frequency Response

The frequency response shows that the repetition rate range used for these tests $(10 \mathrm{kHz}-40 \mathrm{kHz})$ are fully amplified.

\subsection{Calibration}

Since no data was available for the LDTK-028K with respect to low displacement vs. voltage or force vs. voltage, a method for calibration was required. By dropping a very small mass onto the tip of the sensor (in the same fashion as our propulsion occurs), a ratio of calculated force to tested voltage was determined to be $42.35 \mathrm{~V} / \mathrm{N}$. This value is an approximation of the relation as a dropped mass and a repetitive force induced from the laser are very different. The relation is assumed to be a good approximation as the sensitivity was expected to be higher than in the axial tension mode of $14.4 \mathrm{~V} / \mathrm{N}$. 
This was validated using various masses and vertical distances, all samples start from rest on a platform using solely the acceleration of gravity to provide velocity. Impact force can be calculated using Equation (3.12) for Impulse; rearranged in Equation (4.1).

$$
\begin{aligned}
I=\int_{t i}^{t f} F_{\text {impact }} d t & \approx m \Delta V_{\text {impact }} \\
F_{\text {impact }} \Delta t & \approx m \Delta V_{\text {impact }} \\
F_{\text {impact }} & \approx \frac{m \Delta V_{\text {impact }}}{\Delta t}
\end{aligned}
$$

Where $F_{\text {impact }}$ represents the amount of force applied to the surface of the piezoelectric film, $\Delta t$ is the amount of time that mass is in contact with the surface, $\mathrm{m}$ is the mass of the object, and $\Delta V_{\text {impact }}$ is the velocity of the mass at impact, defined by Equation (4.2) below, derived from the equation for work. The time of the impact was estimated to be 0.001 seconds, and was roughly verified with a high speed camera. The high speed camera captured 1000 frames per second and it was verified that the mass was in contact for just a single frame. The error with this assumption is that the impact time could have in fact been faster, but since there is no method for accurate validation available, the slowest time that was attainable from the images was used. Force in the equations below is defined as mass multiplied by gravitational acceleration of the object, $\mathrm{F}=\mathrm{ma}$.

$$
\begin{aligned}
\text { Work } & =F \Delta h \\
& =\int F(h) d h \\
& =\int m \frac{d V}{d t} d h \\
& =m \int \frac{d h}{d t} d V \\
& =\frac{m\left(V_{f}^{2}-V_{i}^{2}\right)}{2} \\
E_{k} & =\frac{m V_{f}^{2}}{2}
\end{aligned}
$$

Where $\mathrm{h}$ is the distance that the mass falls, $V_{f}$ is the final velocity, and $V_{i}$ is initial velocity which has been set to zero as the mass starts from rest in these experiments. Using Equation (4.2), commonly known as the kinetic energy, an energy balance can be used; shown in Equation (4.3) below, commonly known as the gravitational potential energy. 


$$
\begin{aligned}
\text { Work } & =F \Delta h \\
& =m g h
\end{aligned}
$$

Equating Equation (4.2) to Equation (4.3), the final velocity of the mass just before impact can be calculated and is shown in Equation (4.4).

$$
\begin{aligned}
m g h & =\frac{m V_{f}^{2}}{2} \\
V_{f} & =\sqrt{2 g h}
\end{aligned}
$$

Where $\mathrm{g}$ is the gravitational acceleration, and $V_{f}$ describes the final velocity of the mass just before impact $\left(V_{\text {impact }}\right)$. With these equations, it was assumed that the effects of air drag and rotation of the falling mass are negligible to the magnitude of the impact force.

The calibration data should be taken as an approximation of the force to voltage output as the force is very small and could be affected by the amplifier and connecting cables. The signals gathered for the single dropped mass and the highly repetitive pulse forces of the laser ablation are fairly different, which further indicates that this calibration method is not precise. An example of a sinusoidal signal from calibration is given in Figure 4-17.

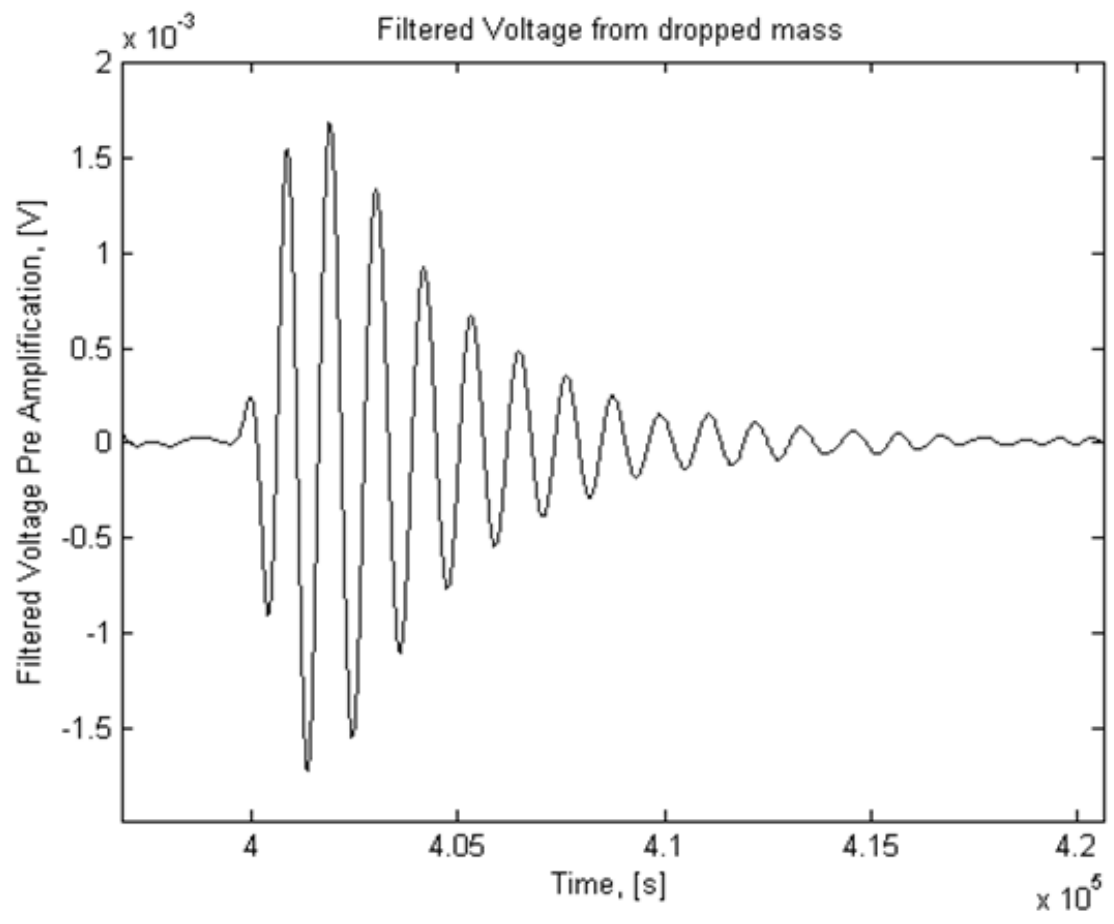

Figure 4-17: Signal from Dropped Mass

Another example given below in Figure 4-18 shows the sensor picking up the mass bouncing several times after impact. 


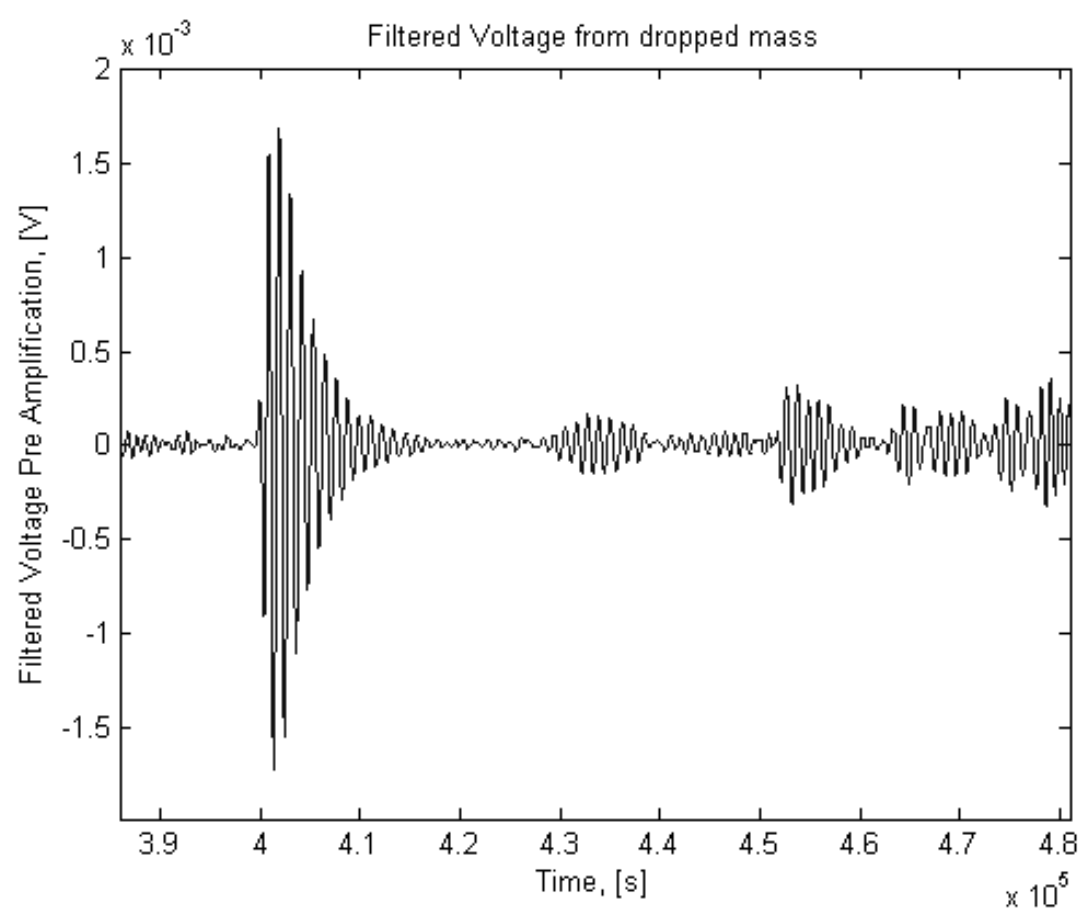

Figure 4-18: Bouncing Calibration Mass

The data is run through the generated MATLAB code and Simulink filter setup, given in Appendix A: MATLAB Code and Simulink Filters. The absolute maximum value of the filtered signal voltage was divided by the calculated force from Equation (4.1) to create a force to voltage ratio $(\mathrm{V} / \mathrm{N})$. The various masses were all used to create an average ratio. The ratio is used to convert all test data from a voltage signal to a force vs. time graph.

\subsection{Summary}

A test setup was developed to evaluate the effects of using a high repetition rate nanosecond laser for laser ablation propulsion. The test setup includes an amplifier system designed specifically for the piezoelectric film used in these tests. The laser was fired repetitively at the same spot to study the effects of using a lower energy repetitive pulse laser for thrust production. Moment coupling coefficient $C_{m}$, and specific impulse $I_{s p}$ values were evaluated as well as the effects of the laser parameters on the propellant. 


\section{Chapter 5: Nanosecond Laser Micropropulsion}

\subsection{Test Setup}

The testing apparatus is setup as shown below in Figure 5-1. Tests were conducted in an isolated room maintained at $20^{\circ} \mathrm{C}$ at $30 \%$ humidity, on an optics table to isolate the testing area from ambient vibrations.

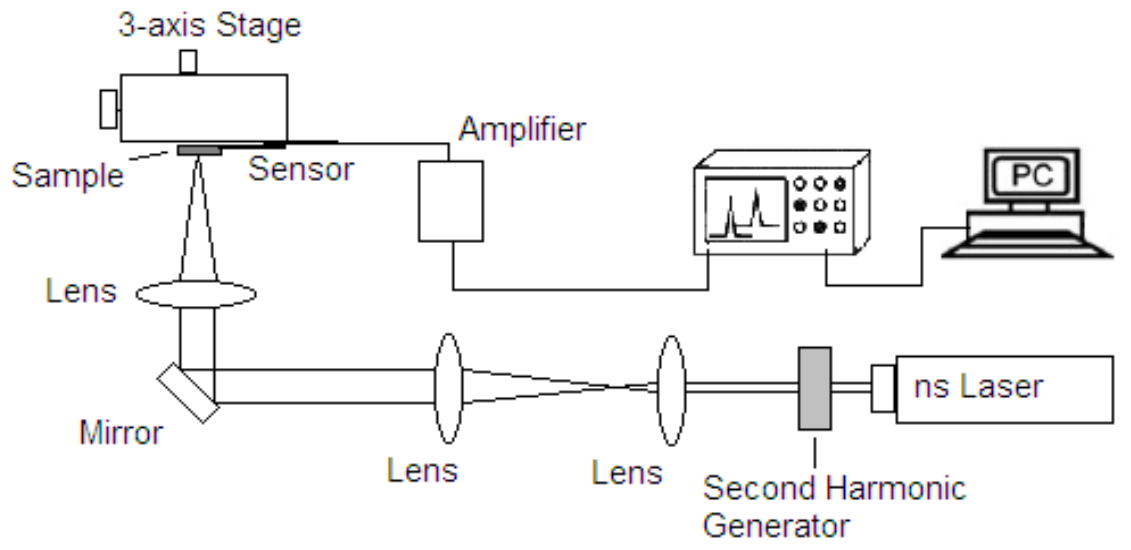

Figure 5-1: Apparatus Layout

The laser source was a commercially available Q-switched diode pumped solid state (DPSS) Nd:YAG industrial laser delivering $10 \mathrm{~W}$ of $1064 \mathrm{~nm}$ light at $10 \mathrm{kHz}$ to $40 \mathrm{kHz}$. The light is passed through a second harmonic generator which outputs two wavelengths of light at $1064 \mathrm{~nm}$ and $532 \mathrm{~nm}$. This is called the dual wavelength effect, which effectively increases the absorption of the laser light. For materials that have high absorption efficiencies around $532 \mathrm{~nm}$, this is very beneficial. The material first absorbs the $532 \mathrm{~nm}$ light and changes the absorption coefficient due to an increased electron density distribution. This change enables the $1064 \mathrm{~nm}$ wavelength to be absorbed more efficiently. Excited electron density and plasma plume density was found to be higher for dual wavelength pulses than for single by Sivakumar et al. [69].

The laser light ablates the propellant attached to the sample, which is then deflected. The signal is received by the amplifier, where the signal is filtered for $1 \mathrm{kHz}$ to $600 \mathrm{kHz}$ and amplified by $1000 \mathrm{x}$. The amplifier outputs the signal to the oscilloscope [LeCroy Wavesurfer 422], which is captured and analyzed using MATLAB on a PC. An image of the testing facility is given below in Figure 5-2. 


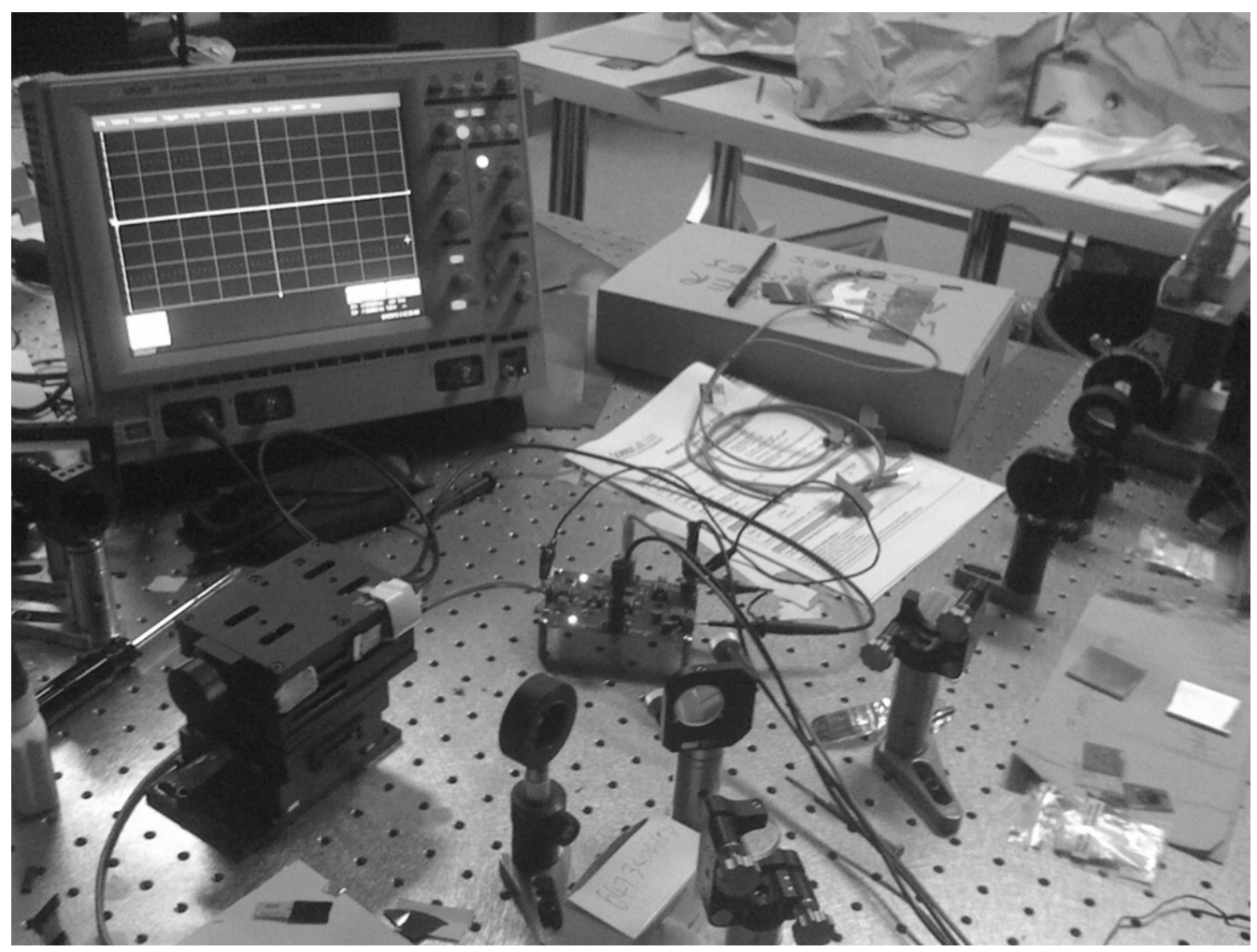

Figure 5-2: Apparatus Setup

\subsection{Propellants}

For initial testing of the system, poly-vinyl chloride (PVC) was chosen as a propellant due to the resources available regarding its behavior during laser ablation. As testing continued other materials were tested to evaluate the effects of high repetition rate materials for use as propellant. Metals were added to the testing as well since they were found in other tests to have high exhaust velocities resulting in high specific impulse values relative to polymers. A list of materials used is given below in Table 5-1.

\begin{tabular}{|l|c|c|c|c|c|c|}
\hline Material & Aluminum & Aluminum & PVC & PVC & Brass & Brass \\
\hline Thickness [in] & 0.005 & 0.005 & 0.065 & 0.0045 & 0.005 & 0.001 \\
\hline Colour & Blue & Natural & Dark Grey & White & Natural & Natural \\
\hline $\begin{array}{l}\text { Thermal } \\
\text { Conductivity } \\
\text { [W/mK] }\end{array}$ & 250 & 250 & 0.19 & 0.19 & 109 & 109 \\
\hline
\end{tabular}

Table 5-1: Materials Used for Testing 
The colour of the material is given as the coloured aluminum was found to produce higher thrust results than the natural aluminum colour. The blue coating is believed to be a thin coat of paint used by the distributor to differentiate the material thicknesses. The aluminum samples are both of the same alloy. The thermal conductivity is given as a comparison for the materials used [70]. The material ablated per pulse is expected to be a reflection of the thermal conductivity. The mass ablated per pulse for PVC is expected to be the highest of the three materials due to the polymer having a low melting temperature of approximately $100^{\circ} \mathrm{C}$ compared to the metals being around $900^{\circ} \mathrm{C}$. This will also strongly contribute to the heat affected zone of the PVC being larger than the other materials.

The samples were all attached to the sensor with an adhesive so that the overlap was on the lamination of the sensor and not on the actual PVDF. This was to reduce the chance of pyroelectric effects and to reduce the chances of de-lamination when the sample was removed from the sensor. The samples were examined to ensure that the bond between the propellant and the sensor was strong enough to be considered rigid for these tests. The adhesive joint is expected to reduce the transferred thrust by a very small amount, assumed to be consistent over all tests and negligible. The propellants were all ablated in the same fashion and same patterning to maintain consistency between results. The effects of ablating the material at different distances from the tip of the sensor were examined for the $0.005^{\prime \prime}$ blue aluminum. A $15 \%$ reduction was noticed in the signal over a 0.2 " distance.

Seeking thrust values, the thickness of the material was not expected to be an issue as the laser was allowed to fully pierce the material during data recording. For these tests it was assumed that the laser ablation occurred in reflective mode due to the thickness and positioning of the material.

\subsection{Digital Filtering}

The signal output from the piezofilm was quite noisy due to atmospheric interference, amplifier noise, etc. In addition, and most importantly to understand is the signal-to-noise ratio (SNR) was very low; the voltage output from the sensor due to the small thrust produced was on the order of tens of microvolts. Post process digital filtering of the signal was used to significantly reduce the ambient noise and extract the voltage signal that described the interaction of the laser with the propellant. Simulink and MATLAB software was used to post process the data; given in Appendix A: MATLAB Code and Simulink Filters.

A filtering sequence was developed that allowed the thrust signal to be extracted from the raw voltage data. An example of an unfiltered signal to a filtered signal is shown below in Figure 5-3. 


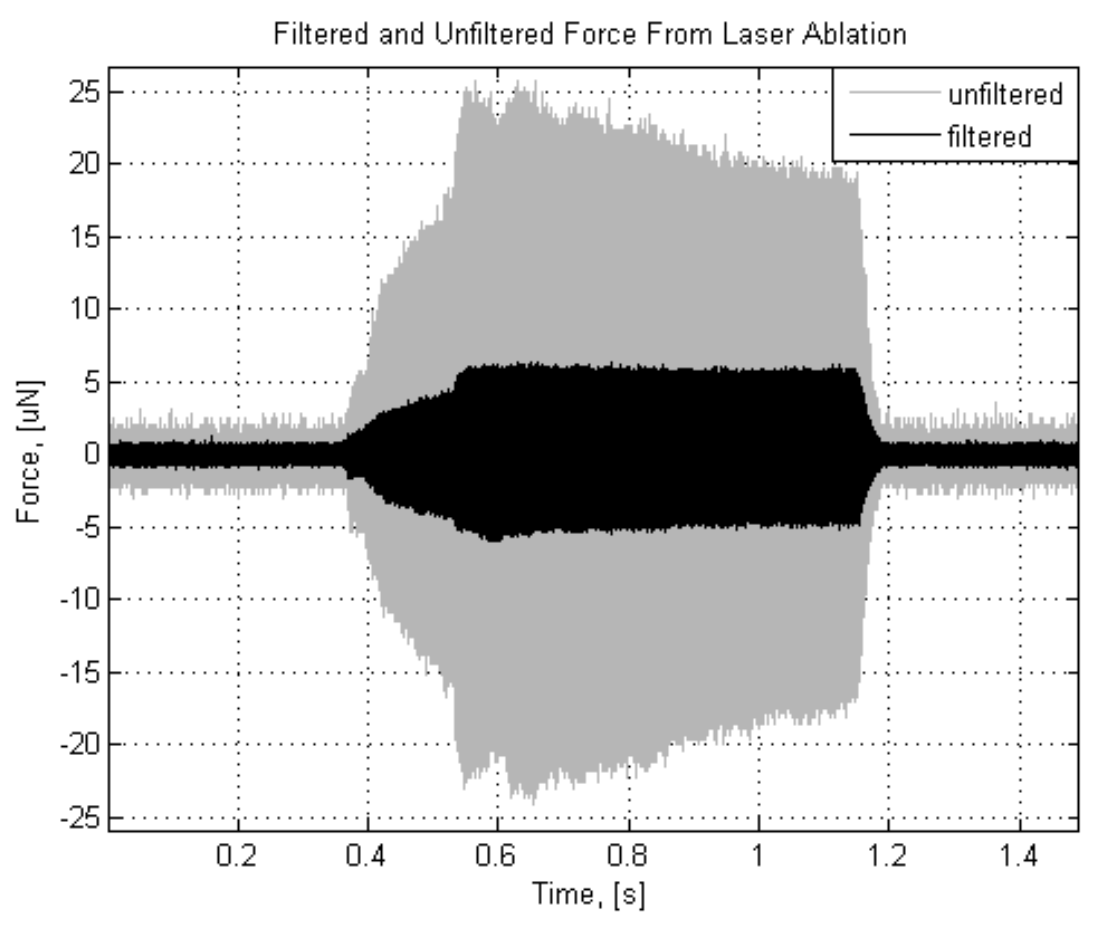

Figure 5-3: Unfiltered and Filtered Signals for Brass, $0.001 ", 121 \mu \mathrm{J}$ at $10 \mathrm{kHz}$

The signal was filtered with two sequential Butterworth type filters. The first filter is a high pass filter with a cut off frequency of $8 \mathrm{kHz}$, coupled to a notch type that cuts out frequencies between $45 \mathrm{kHz}$ and $200 \mathrm{kHz}$. The combination was used instead of a bandpass filter due to the plasma plume expansion occurring over several microseconds. By keeping the upper frequencies in the signal, the neutral signal noise did not increase but the signal during ablation increased slightly. The signal in Figure 5-3 shows how the noise signal is reduced. The filtering effects differ slightly over the range of materials and parameters, which is unavoidable if any filtering is used. The same signal using only a bandpass filter between $8 \mathrm{kHz}$ and $20 \mathrm{kHz}$ is shown below in Figure 5-4 for comparison of the filtering methods. 


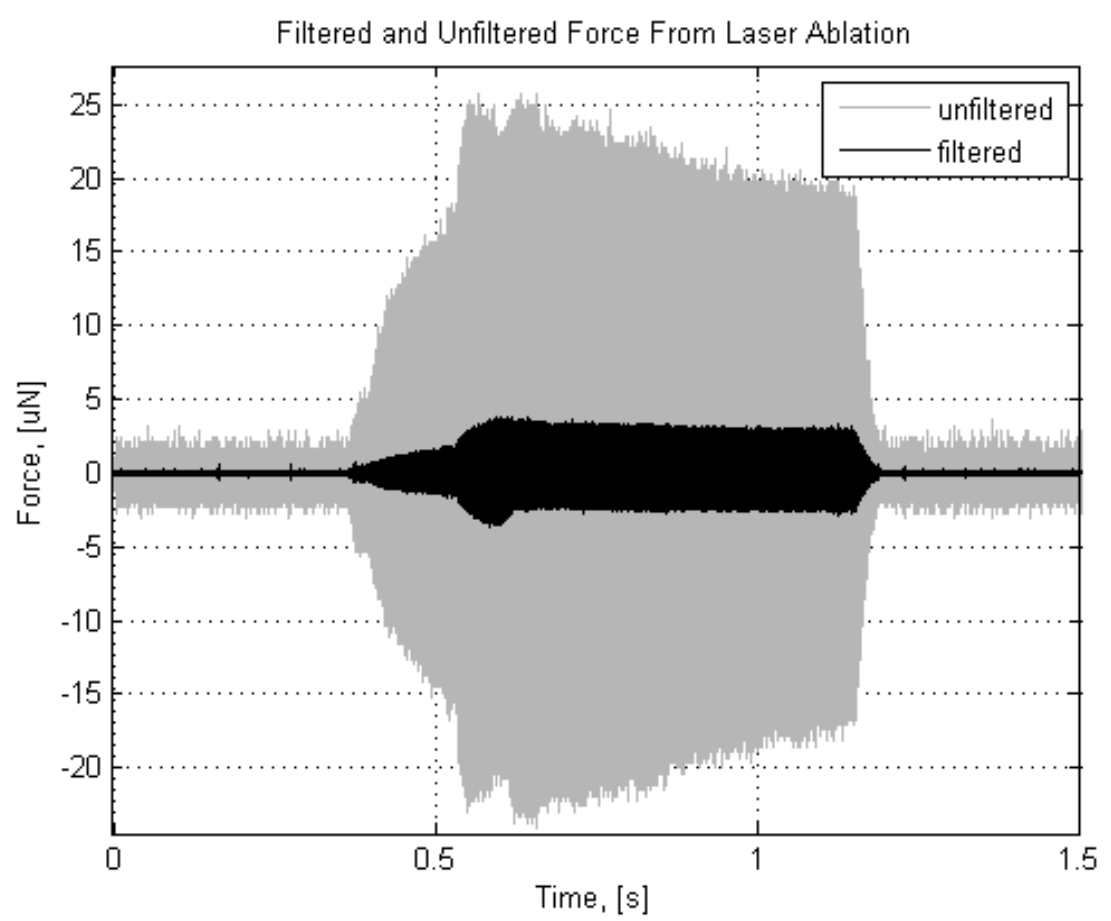

Figure 5-4: Unfiltered on Filtered Signals for Brass, $0.001 ", 121 \mu \mathrm{J}$ at $10 \mathrm{kHz}$ using bandpass filter

The signal filtered through a bandpass filter for the frequency of the repetition rate shows a larger decrease in the noise signal; however the amplitude of the ablation signal was also greatly affected. The relative difference in the ablation signals between Figure 5-3 and Figure 5-4 is minimal compared to the original signal and as a result the high pass combined with the notch filter was used to extract the thrust signal from the raw data.

\subsection{Results and Discussions}

The testing conducted aimed at comparing the effects that repetition rate and laser pulse energy have on micropropulsion. During testing, several other phenomena relating to ablation and laser material interaction were observed and are discussed in this section.

The depth of focus for these tests was 1.04 [cm]; when considering the $1064 \mathrm{~nm}$ wavelength. As long as the material was within this depth of focus, the focal point position would not contribute much more to the data and power increase is relatively negligible. The focal spot size (using Equation (3.4)) for these tests is $84.45 \mu \mathrm{m}$ at a focal length of $125 \mathrm{~mm}$, with a beam diameter of $2 \mathrm{~mm}$ and wavelength of $1064 \mathrm{~nm}$. Considering that the dual wavelength effect may have had an effect on the spot size, the spot size for $532 \mathrm{~nm}$ was calculated as $42.23 \mu \mathrm{m}$. The spot size effect on different materials is evaluated as well in the 
Laser Material Interaction section of this Chapter. The laser was focused using the visible $532 \mathrm{~nm}$ wavelength focal spot, which is expected to be within the depth of focus of the $1064 \mathrm{~nm}$ wavelength. To focus the laser, an aluminum foil was used at $125 \mathrm{~mm}$ and translated along the beam axis. The foil was observed for sparking in the depth of focus until a strong ablation spot was found. The piezo film with the attached sample was placed at this point and checked again for laser focus.

Maintaining consistency between tests was a priority. Ablation locations for $10 \mathrm{kHz}, 20 \mathrm{kHz}$, and $30 \mathrm{kHz}$ were kept within the same zone of the samples. Sample weight may have impacted sensor response. Samples were kept to approximately $0.5^{\prime \prime}$ by $0.2^{\prime \prime}$. The samples were ablated from top to bottom for each repetition rate, separating each series horizontally by approximately $0.03 "$. The previously mentioned compensation for distance of ablation from the sensor was not added to the results as the actual separations are considered negligible for these discussions.

\subsubsection{Laser Parameters Used for Testing}

The data used for the graphs in this chapter are averaged values of multiple tests at each pulse energy and repetition rate. Standard deviations for the data are given in Appendix C: Test Data Compilation.

Testing was conducted with constant laser parameters given below in Table 5-2.

\begin{tabular}{|l|c|c|c|}
\hline Repetition Rate & $10 \mathrm{kHz}$ & $20 \mathrm{kHz}$ & $30 \mathrm{kHz}$ \\
\hline Pulse Energy Setting $1 \mu \mathrm{J} /$ pulse & 121 & 120 & 115 \\
\hline Pulse Energy Setting $2 \mu \mathrm{J} /$ pulse & 185 & 180 & 162 \\
\hline Pulse Energy Setting $3 \mu \mathrm{J} /$ pulse & 237 & 237 & 207 \\
\hline
\end{tabular}

Table 5-2: Laser Parameters for Tests

It is interesting to note that the pulse energies are the same at $10 \mathrm{kHz}$ and $20 \mathrm{kHz}$ due to the average power doubling as well as the repetition rate. The effects would be expected to produce the same results since the repetition rates are both greatly lower that would promote LSD, which would be near $1 \mathrm{MHz}$. Originally, the $10 \mathrm{kHz}$ repetition rate was expected to produce higher thrust values. The effects of the repetition rate may only affect the pulse energy and not the thrust production as a result.

\subsubsection{Waveform Patterns}

Patterns in the waveforms were observed at the different repetition rates. The zoomed in waveforms were observed at $10 \mathrm{kHz}, 20 \mathrm{kHz}$, and $30 \mathrm{kHz}$, shown below in Figure 5-5, Figure 5-6, and Figure 5-7 respectively. Enlarged images are given to demonstrate the timescale of the occurrences. 


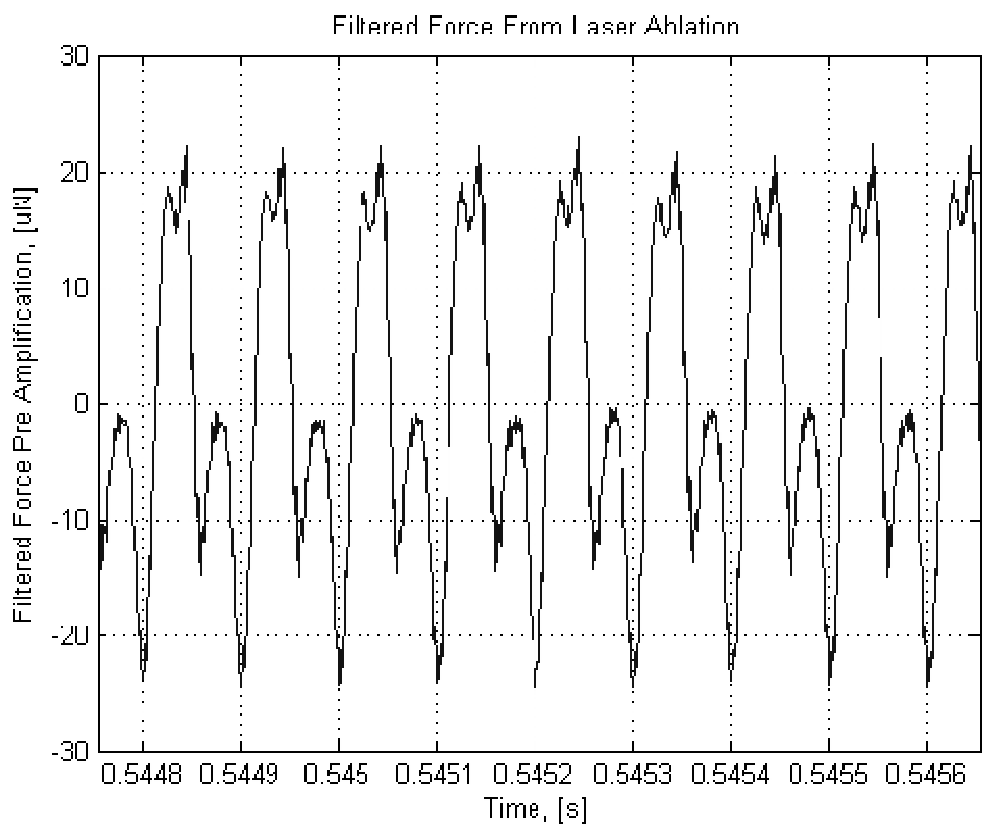

Figure 5-5: Brass $10 \mathrm{kHz} 238 \mu \mathrm{J}$

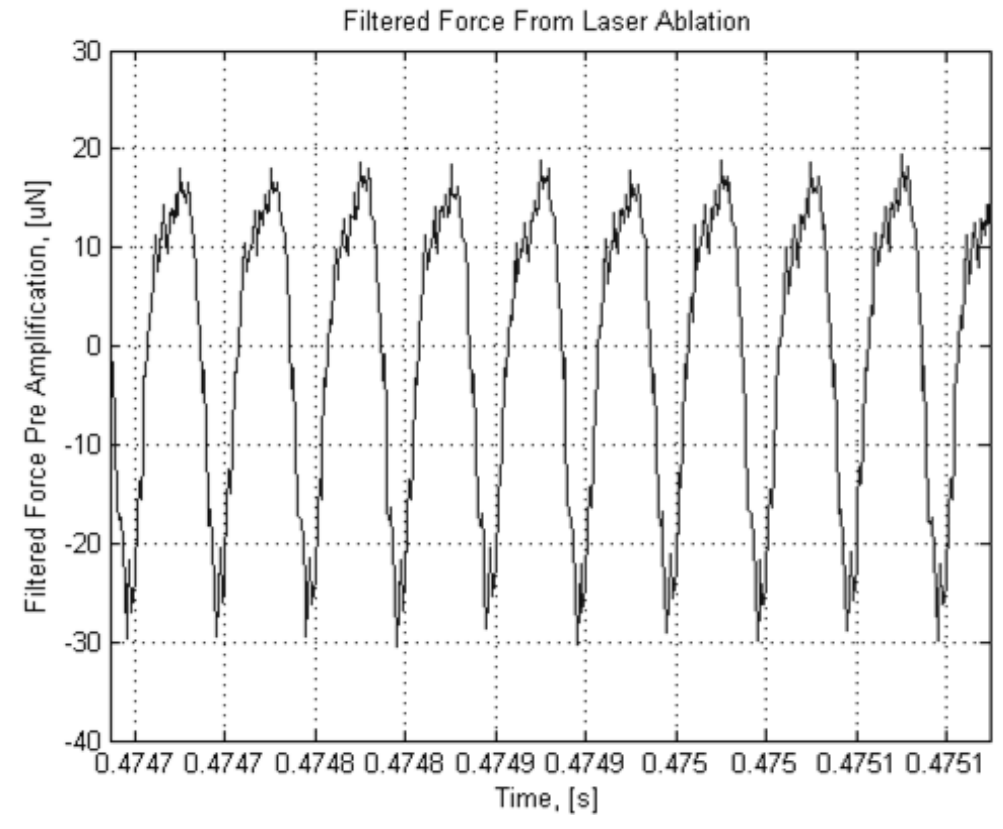

Figure 5-6: Brass 20kHz 238 $\mu$ J 


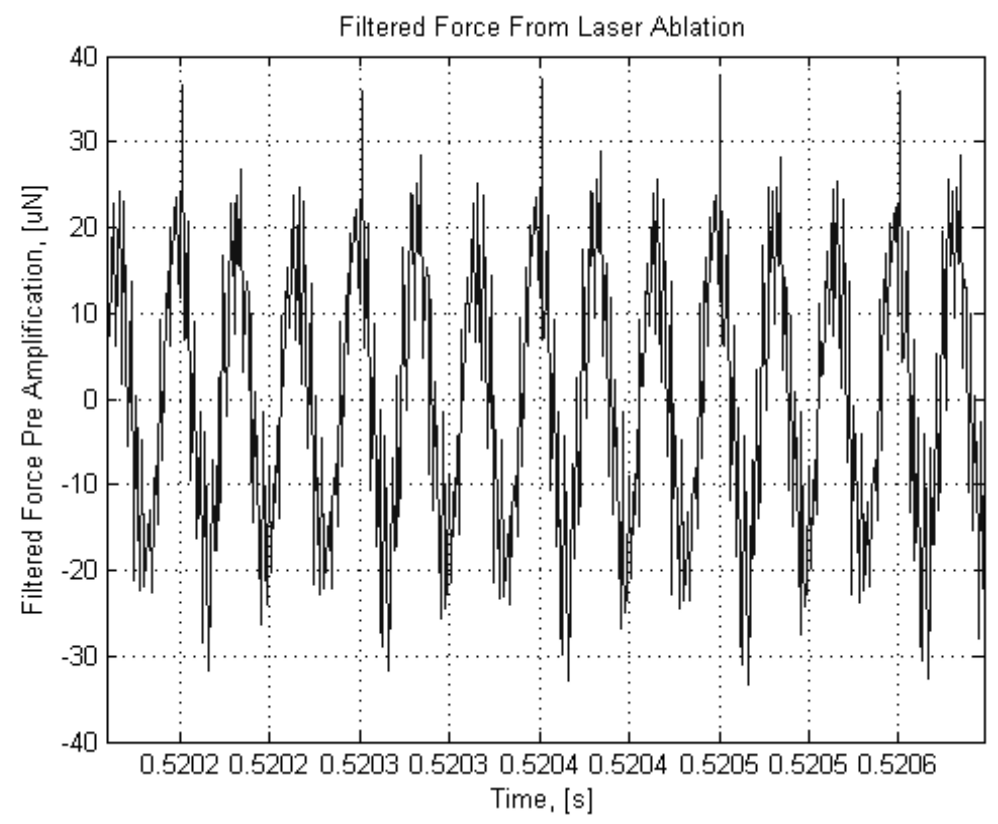

Figure 5-7: Brass $30 \mathrm{kHz} 207 \mu \mathrm{J}$

At $10 \mathrm{kHz}$ multiple waveforms are present in the signal, which was assumed to be thrust and a rebounding response of the piezoelectric film. As was observed in the calibration waveform (Figure 4-17), in the system response to an impulse there is a small artifact before the peak, and then a gradual signal dampening. Naturally, the frequency of the impulses significantly changes this behavior, however, at $10 \mathrm{kHz}$ it responds very similarly. At $20 \mathrm{kHz}$ and $30 \mathrm{kHz}$ there are no similarities to the $10 \mathrm{kHz}$ signal. Between the $20 \mathrm{kHz}$ and $30 \mathrm{kHz}$ signal the similarity is a consistent wave with no evidence of an overlapping waveform. Each peak occurs exactly at the repetition rate for the $20 \mathrm{kHz}$ and $30 \mathrm{kHz}$ signal. This suggests that the system is moving harmonically with the force produced via laser ablation for $20 \mathrm{kHz}$ and $30 \mathrm{kHz}$. The $10 \mathrm{kHz}$ signal also responded with the repetition rate, as each major peak is at the $0.0001 \mathrm{~s}$ marker. This occurrence was fairly common with the signals obtained.

\subsubsection{Laser Power and Repetition Rate}

Pulse energy is believed to be the greatest contributor to laser propulsion. The intensity of the laser power can be greatly increased by reducing the spot size of the focal point, or increasing the pulse energy. A smaller focal point may not significantly increase thrust produced but may increase specific impulse, since less mass with a higher velocity is expected from the ablation. Pulse width has also been shown to significantly change the ablation dynamics. The pulse width for these tests was not changed. In this section the maximum thrust for each test recorded is averaged and compared against pulse 
energy at the respective repetition rate. Figure 5-8 shows a thrust vs. pulse energy graph, in units $\mu \mathrm{N}$ and $\mu \mathrm{J} /$ pulse respectively, for the two aluminum materials tested.

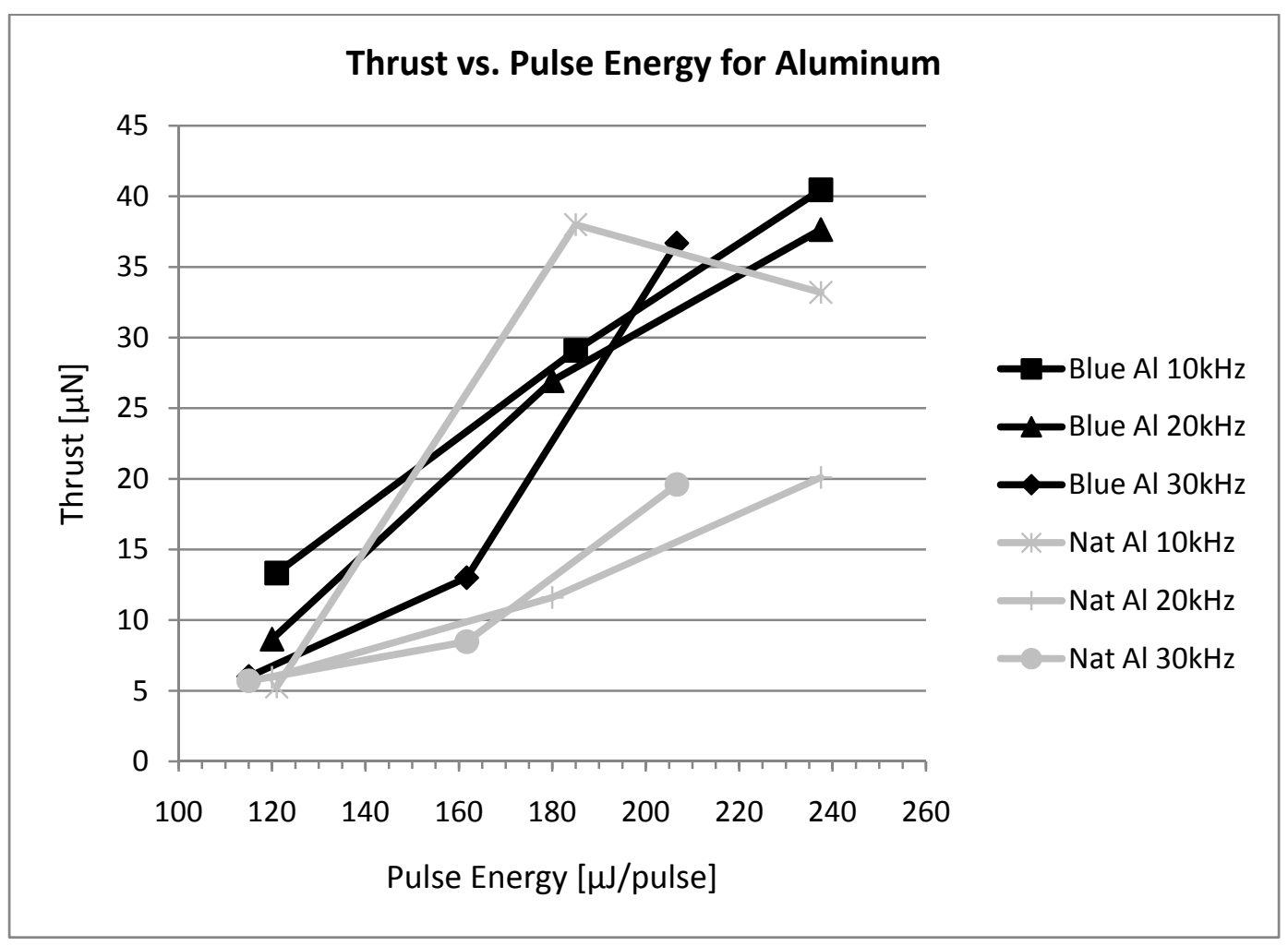

Figure 5-8: Thrust vs. Pulse Energy for Aluminum

For aluminum the difference in thrust output is fairly significant between the blue coated aluminum and the natural aluminum. The data at $10 \mathrm{kHz}$ for the natural aluminum does not follow the trend and could potentially be erroneous data, specifically considering that at a higher pulse energy it is expected to generate more thrust. The data for all test points is given in Appendix C: Test Data Compilation. The previous assumption that the $10 \mathrm{kHz}$ and $20 \mathrm{kHz}$ repetition rates would produce similar thrust levels is true for the blue aluminum sample. The effects of the repetition rate on specific impulse will be described in the next section.

The thrust vs. pulse energy graph for PVC, is given below in Figure 5-9. 


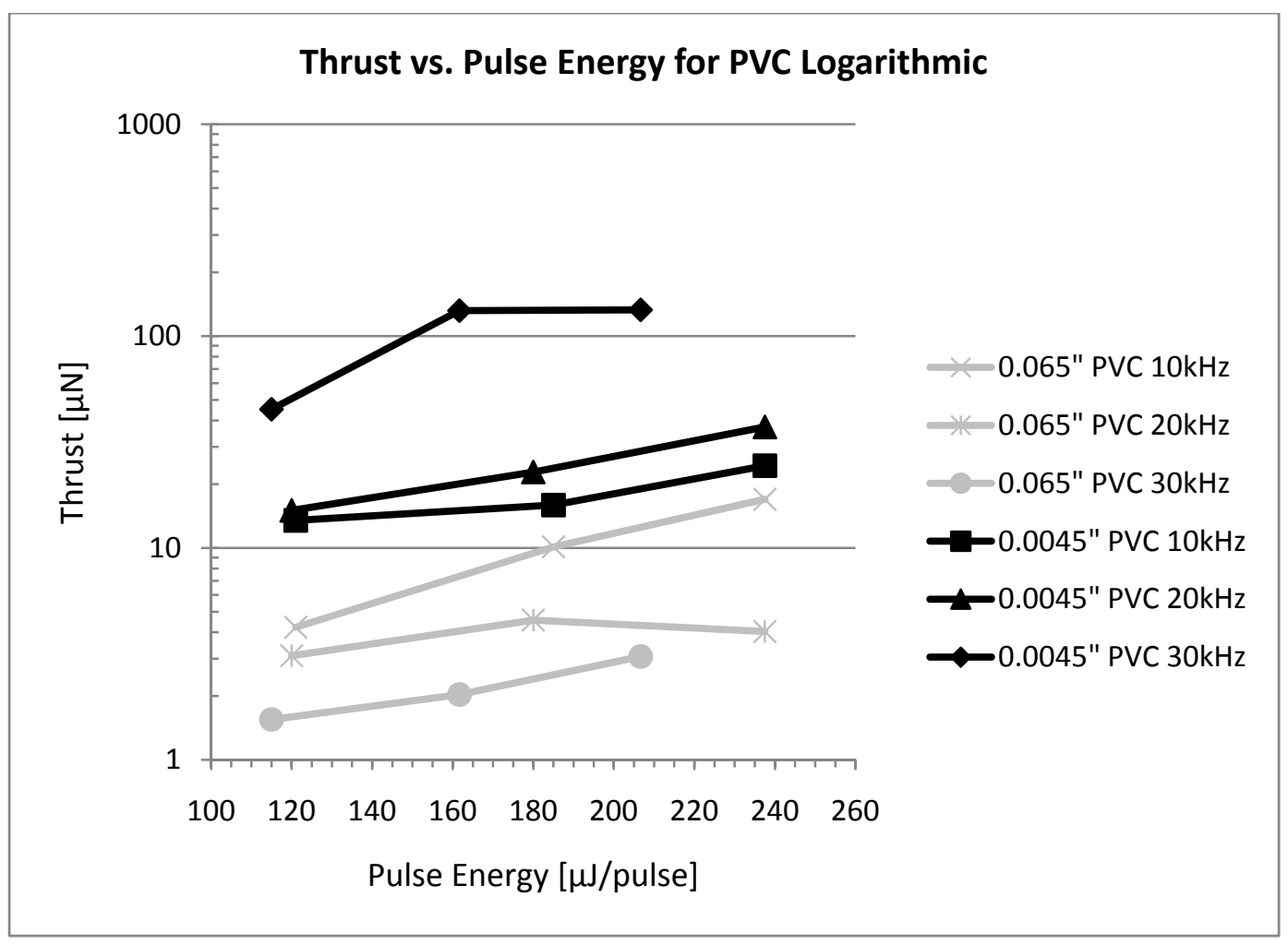

Figure 5-9: Thrust vs. Pulse Energy for PVC Logarithmic

For the PVC graph a logarithmic scale was chosen due to one set of data being significantly higher (almost a full order of magnitude) than the rest of the data. These values range from $45 \mu \mathrm{N}$ to $145 \mu \mathrm{N}$. This sample was attached to a sheet of glass, so the pyroelectric effect is not believed to have any influence in this case since the material was consumed so quickly and due to the thickness of the glass relative to the sample. The sample was placed on glass because it was not very rigid at $0.0045^{\prime \prime}$.

The consistently high thrust values for the $0.0045^{\prime \prime}$ sample at $30 \mathrm{kHz}$ could be due to the thinner material ejecting a higher amount of mass per pulse, or could be due to incubation. The mass ablated per pulse is shown later in this chapter in Table 5-4: Max $C_{m}$ and $I_{s p}$ for each Material. Polymers typically encounter incubation during laser ablation, which is where the fluence threshold of the material decreases with successive pulses. The higher repetition rates for the thinner material could be promoting this effect. The 0.065 " PVC sample does not seem to follow the same trend, which could be completely influenced by the thickness of the material promoting energy loss to melting. The $0.0045^{\prime \prime}$ PVC has less surrounding material volume to conduct heat away from the ablation zone, which could be contributing to the high thrust data. The PVC has a low thermal conductivity, however the melting of 
the material is considered due to the thermal energy transfer to the surrounding material. The PVC data is given below in Figure 5-10 without the $30 \mathrm{kHz}$ data for the $0.0045^{\prime \prime}$

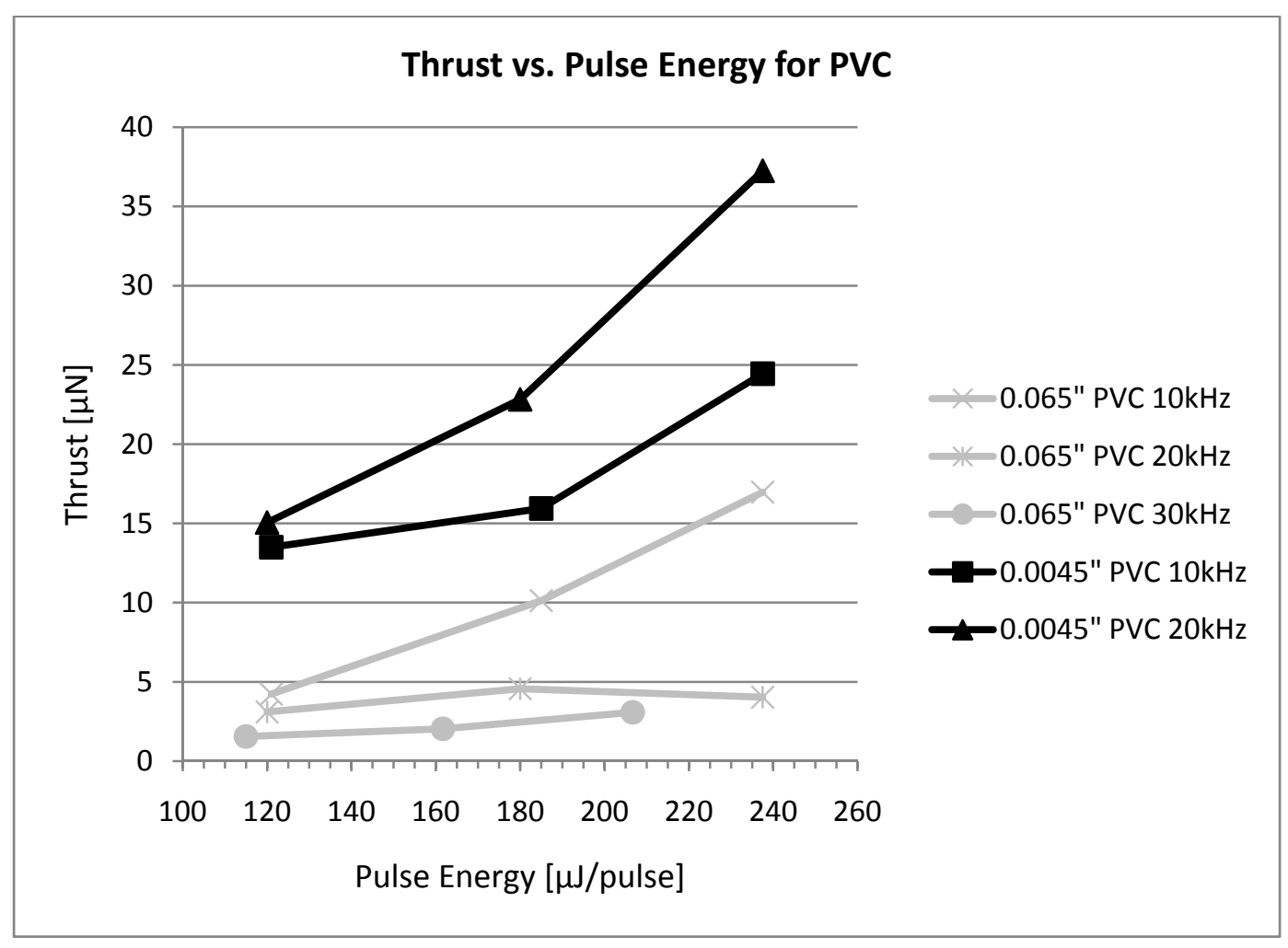

Figure 5-10: Thrust vs. Pulse Energy PVC w/o 0.0045 at 30khz

The data trend for the $0.065^{\prime \prime}$ sample is as expected, and is again similar to the brass and aluminum results.

The thrust vs. pulse energy graph for brass is given below in Figure 5-11. 


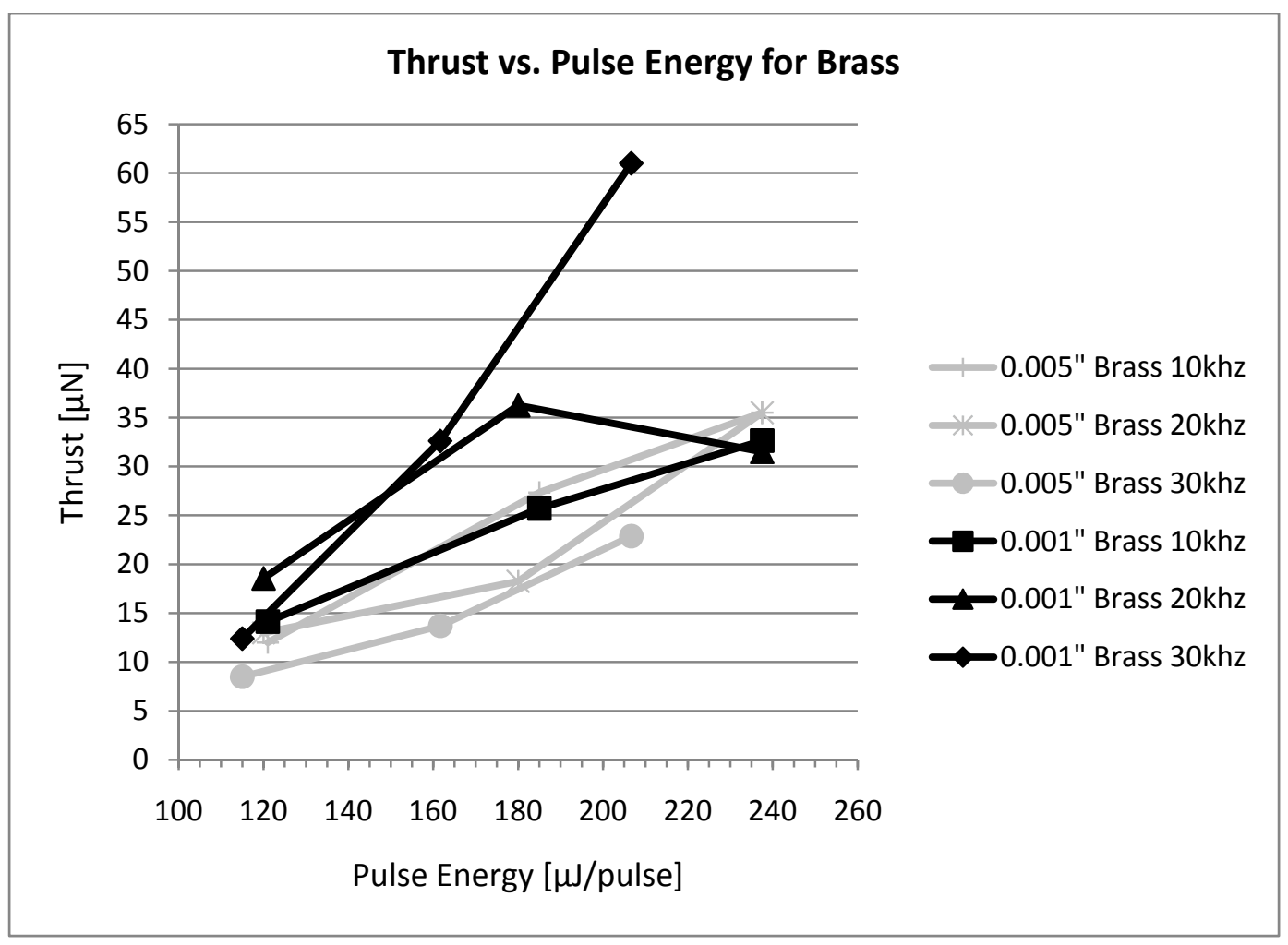

Figure 5-11: Thrust vs. Pulse Energy for Brass

The data for the 0.001 " brass is very sporadic and is potentially due to the thinner material convecting heat to the sensor, so the spike in the results may be attributed to pyroelectricity. As pyroelectric effects are not desired in these tests the system was designed to minimize pyroelectric signals, but in this case the thin material could have contributed. As the resonance frequency of the system is unknown, the higher repetition rates with the thinner materials could be in resonance as well. The signals from the $0.005^{\prime \prime}$ sample are predictable and are very similar in trend and magnitude to the aluminum samples.

Tabulated data of the max thrust for each sample is given below in Table 5-3.

\begin{tabular}{|l|c|c|c|c|c|c|}
\hline Material & $\begin{array}{c}\text { Aluminum } \\
\text { Blue }\end{array}$ & $\begin{array}{c}\text { Aluminum } \\
\text { Natural }\end{array}$ & PVC 0.065" & PVC 0.0045" & Brass 0.005” & Brass 0.001" \\
\hline Thrust $[\mu \mathrm{N}]$ & $40.5 @ 10 \mathrm{kHz}$ & $38 @ 10 \mathrm{kHz}$ & $17 @ 10 \mathrm{kHz}$ & $133 @ 30 \mathrm{kHz}$ & $35.5 @ 10 \mathrm{kHz}$ & $61 @ 30 \mathrm{kHz}$ \\
\hline
\end{tabular}

Table 5-3: Max Thrust for each Material

The tabulated thrust values show the magnitude of the thrust production from the thinner materials. The natural aluminum also shows a very minor difference in this case, however on average the natural 
aluminum had much lower thrust values than the blue coated aluminum. This is assumed to be due to an increase in light absorption with the blue coating which stimulated ablation. The table shows a trend in material thickness contributing significantly to thrust and repetition rate effects. The higher repetition rate for both the brass and PVC provided higher thrust results which could be highly dependent on the heat affected zone of the material. The loss of energy to nearby material by convection or due to melting could be decreasing the amount of material ablated per pulse which would reduce the thrust generated.

The data also relates well with those tabulated by Phipps et al.[15] who captured $0.94 \mu \mathrm{N}$ of thrust with an aluminum sample with a laser intensity of $76 \mathrm{TW} / \mathrm{m}^{2}$, and pulse energy of $20 \mu \mathrm{J}$ at $8 \mathrm{kHz}$.

\subsubsection{Moment Coupling Coefficient and Specific Impulse}

The moment coupling coefficient and specific impulse are often used as comparison terms for laser micropropulsion. In this section these variables are evaluated compared to laser intensity as it is highly dependent on pulse energy, spot area, and pulse width, which are all parameters that significantly differ these tests from those published.

\subsubsection{Ablation Time}

In order to calculate data for this section, a time of generated thrust is required to integrate over, and an ablated propellant mass per pulse is also required. As the propellants all react differently with the laser energy, ablation times were calculated for each propellant at each repetition rate. Originally it was believed that the laser pulse energy would significantly change the amount of time required to pierce through the material fully, however, it was observed that the piercing times are consistent over the pulse energies used for each repetition rate. The piercing time was approximated from the signals gathered during testing. The signals tended to have a pattern where an initial high thrust waveform is noticed, which then tapers to a level until the material is fully removed or until the laser is shut off. Examples are presented below in Figure 5-12. 

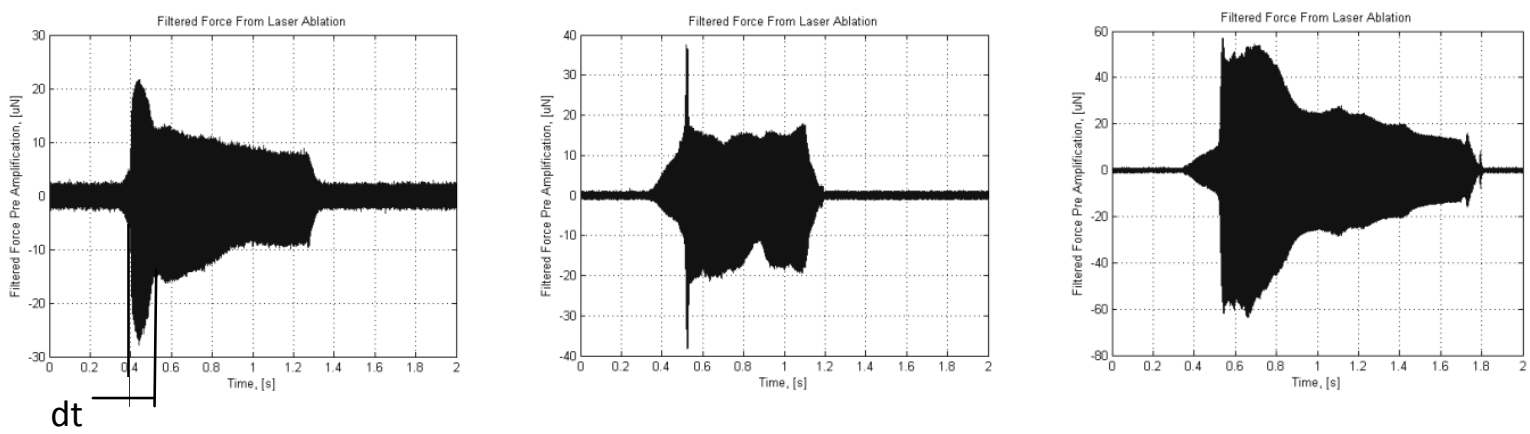

Figure 5-12: Piercing of Brass at (from left to right) $10 \mathrm{khz}, 20 \mathrm{kHz}$, and $30 \mathrm{kHz}$

The elapsed time of the hump (dt) (see Figure 5-10) was used to approximate the amount of time for the laser spot to fully penetrate the material. The continuing signal was assumed to be due to the lower intensity outer ring of the laser spot continuing to ablate the material. This phenomenon was observed in every signal obtained where the material was fully ablated. In the event that the pulse energy was not high enough to fully pierce the material in a short $(<1 s)$ period of time, then the signal often seemed to gradually rise with no sudden peaks or drops until the laser was shut off. An example of this occurrence is given in Figure 5-13.

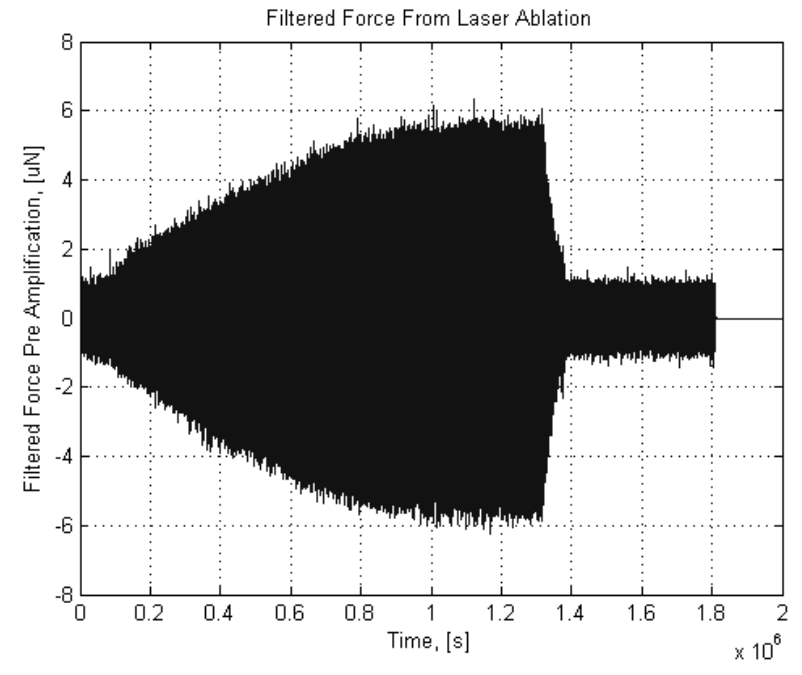

Figure 5-13: $30 \mathrm{kHz} 115 \mu$ on Aluminum

The cause of this signal could have been due to a resonating signal in the system or a pyroelectric effect and should be further investigated in future work.

The Impulse of each test was calculated using the maximum thrust value of each signal and dividing it by two to approximate an average over the half wavelength. The amount of time that the thrust was 
generated was approximated to be $1.5 \mu \mathrm{s}$. This was taken from Pakhomov et al. assumptions that the plasma plume generates thrust for its visible lifetime, which they approximated to $1.5 \mu \mathrm{s}$ [18].

The moment coupling coefficient vs. laser intensity for aluminum is shown below in Figure 5-14.

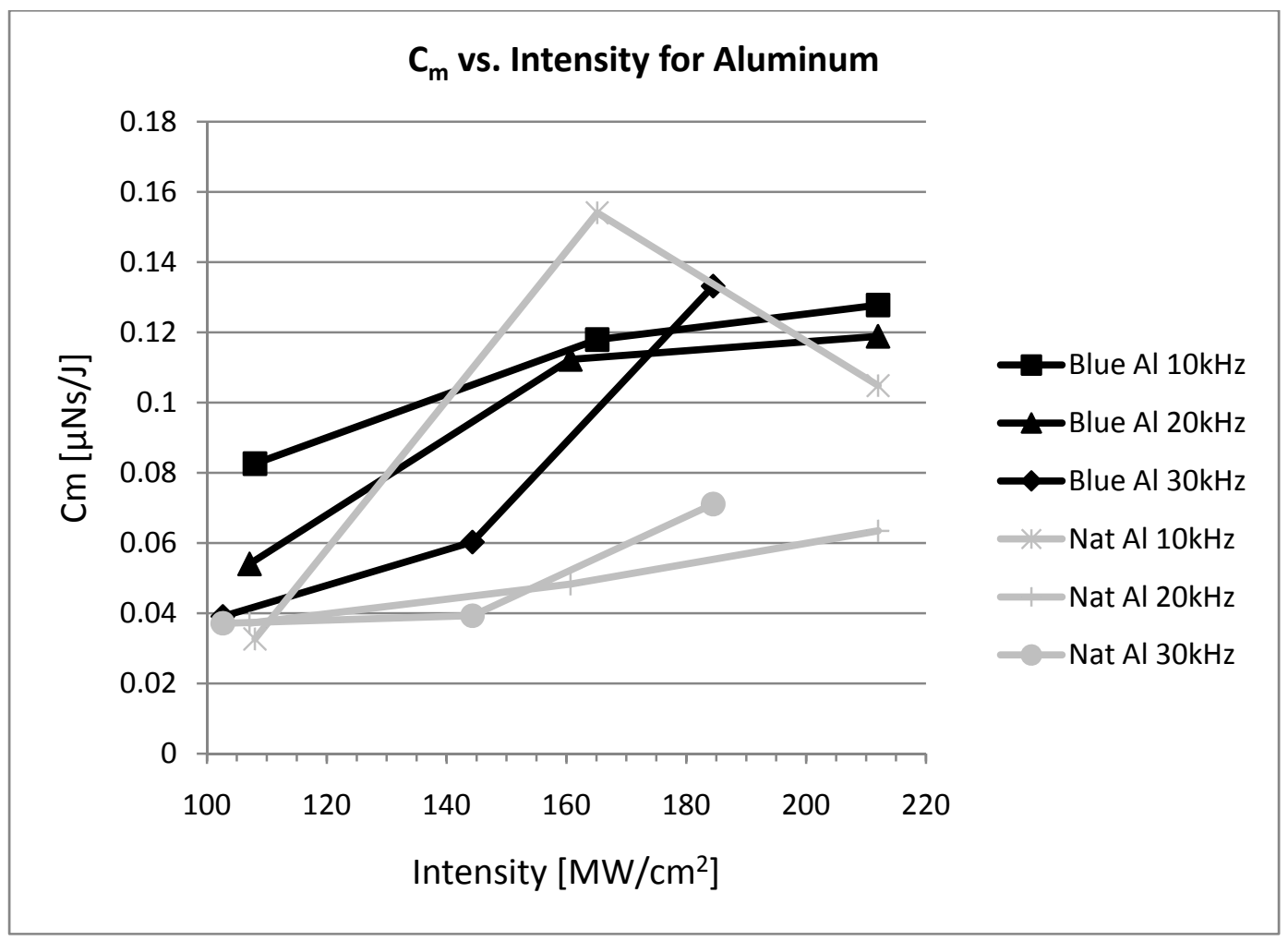

Figure 5-14: $C_{m}$ vs. Intensity for Aluminum

The $C_{m}$ trends are very similar to the thrust trends for the aluminum samples. This data is comparable to those published by Pakhomov et al. who used $8 \mathrm{~mJ}$ pulses at approximately $6.85 \mathrm{TW} / \mathrm{cm}^{2}$ (approximated from their pulse energy reduction between tests) to produce $20 \mu \mathrm{N} / \mathrm{W}$ to $80 \mu \mathrm{N} / \mathrm{W}$ moment coupling coefficients for various metals, including aluminum [18]. The difference between the pulse energies is approximately two orders of magnitude, which is approximately the difference between this data and those by Pakhomov et al.

The moment coupling coefficient vs. laser intensity for PVC is shown below in Figure 5-15. 


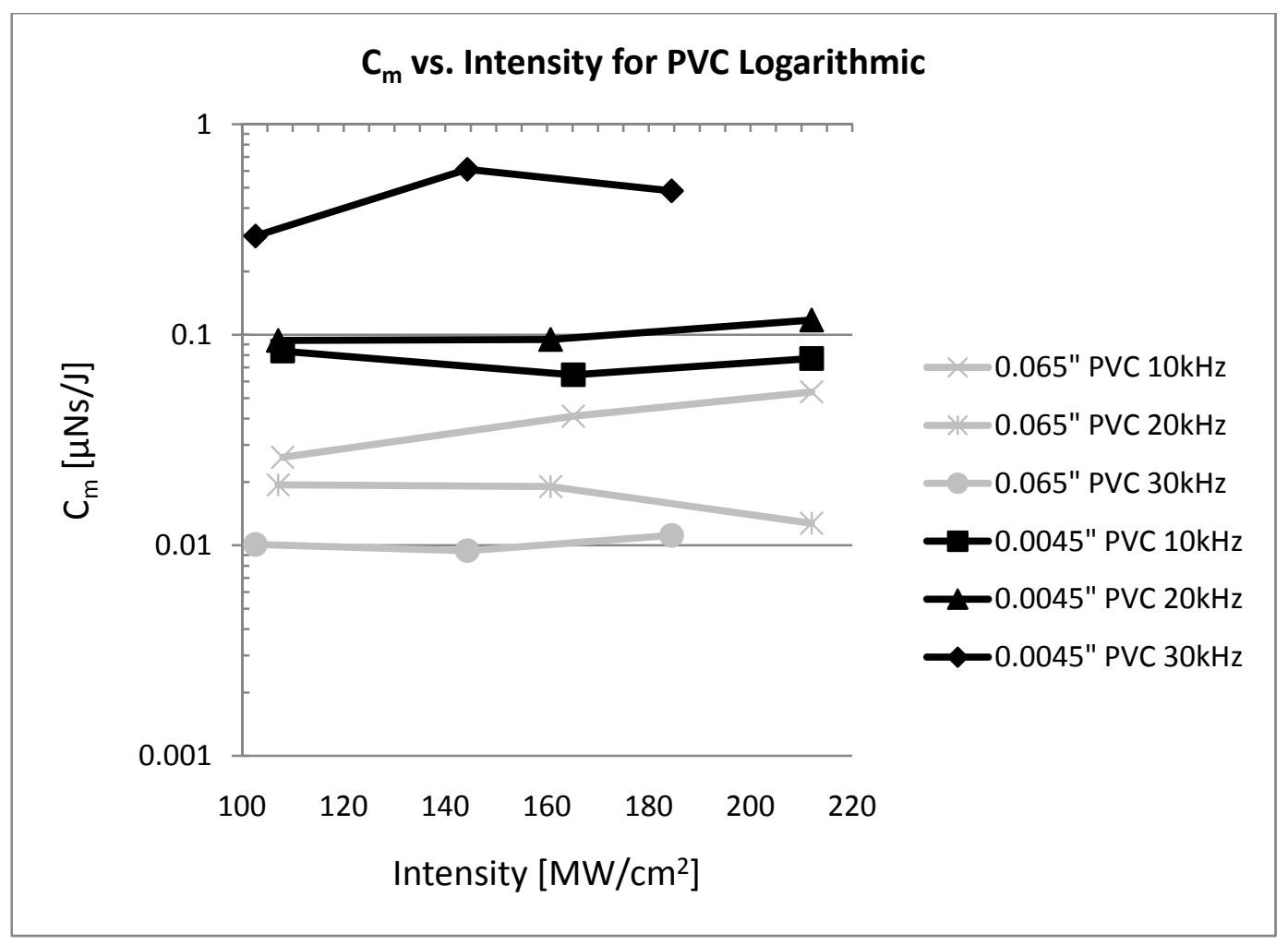

Figure 5-15: Logarithmic $C_{m}$ vs. Intensity for PVC

PVC also follows a similar trend to the thrust data, which was expected. A logarithmic graph was given due to the large difference in $C_{m}$ magnitude between thicknesses. Data for the $0.0045^{\prime \prime}$ PVC samples could be erroneous due to the ablation spot being approximated at lower pulse energies for each repetition rate. The ablated spot area was captured via SEM for the highest pulse energy setting only. This explains why the lower intensity levels for the 0.0045 " PVC are presenting high $C_{m}$ values.

The moment coupling coefficient vs. laser intensity for brass is shown below in Figure 5-16. 


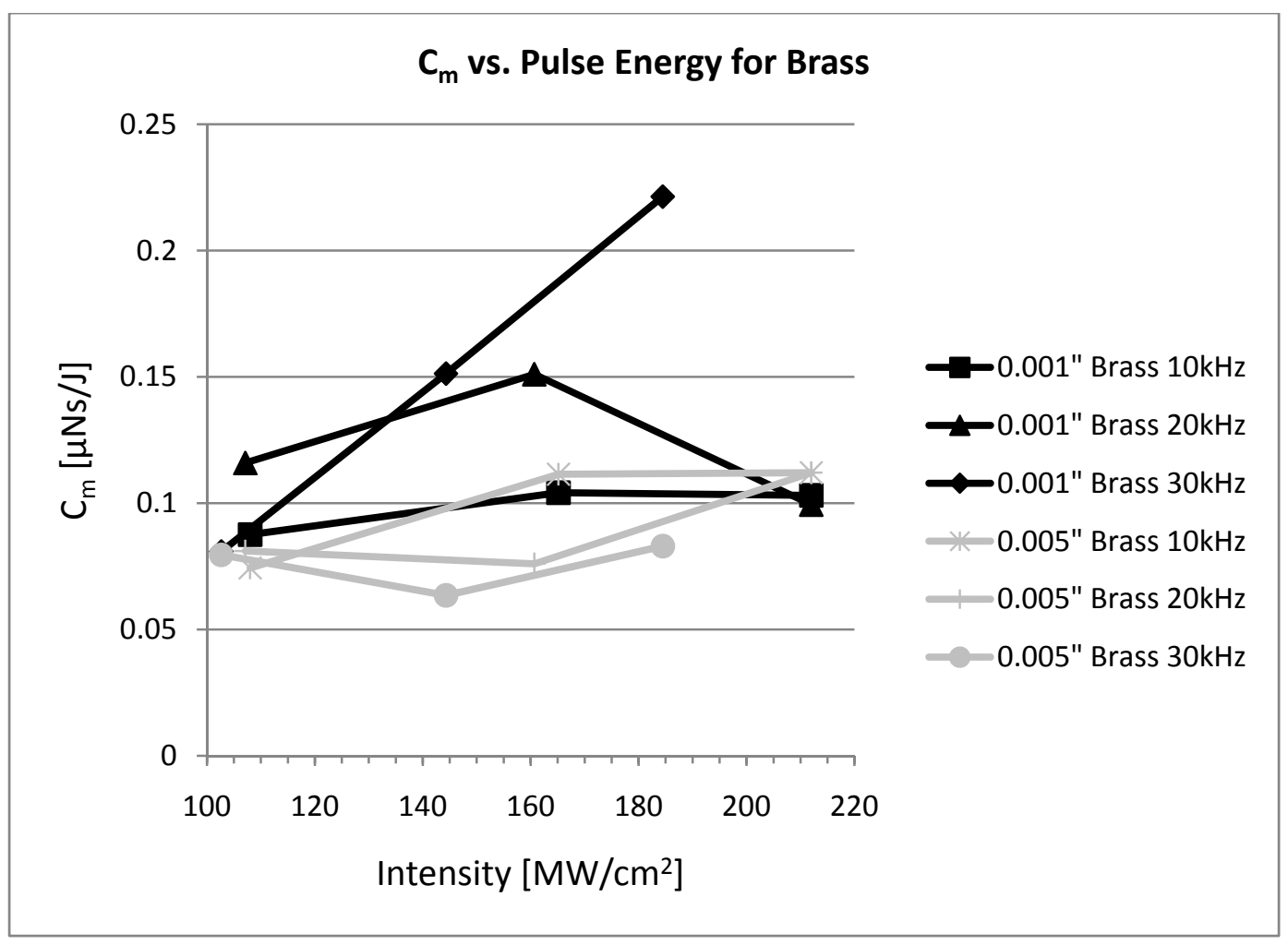

Figure 5-16: $C_{m}$ vs. Intensity for Brass

This follows a similar trend to the thrust graph in the previous section for brass. Brass and aluminum proved to have fairly high $C_{m}$ values but were expected to produce lower $C_{m}$ values than the PVC. The PVC was expected to have the highest $C_{m}$ values of the group. It is assumed that if the actual ablated spot size was determined neglecting the melted material then the $C_{m}$ would be much higher. However, the melted/HAZ material is part of the micropropulsion equations taking into account the amount of material used per thrust produced.

The specific impulse vs. laser intensity for aluminum is shown below in Figure 5-17. 


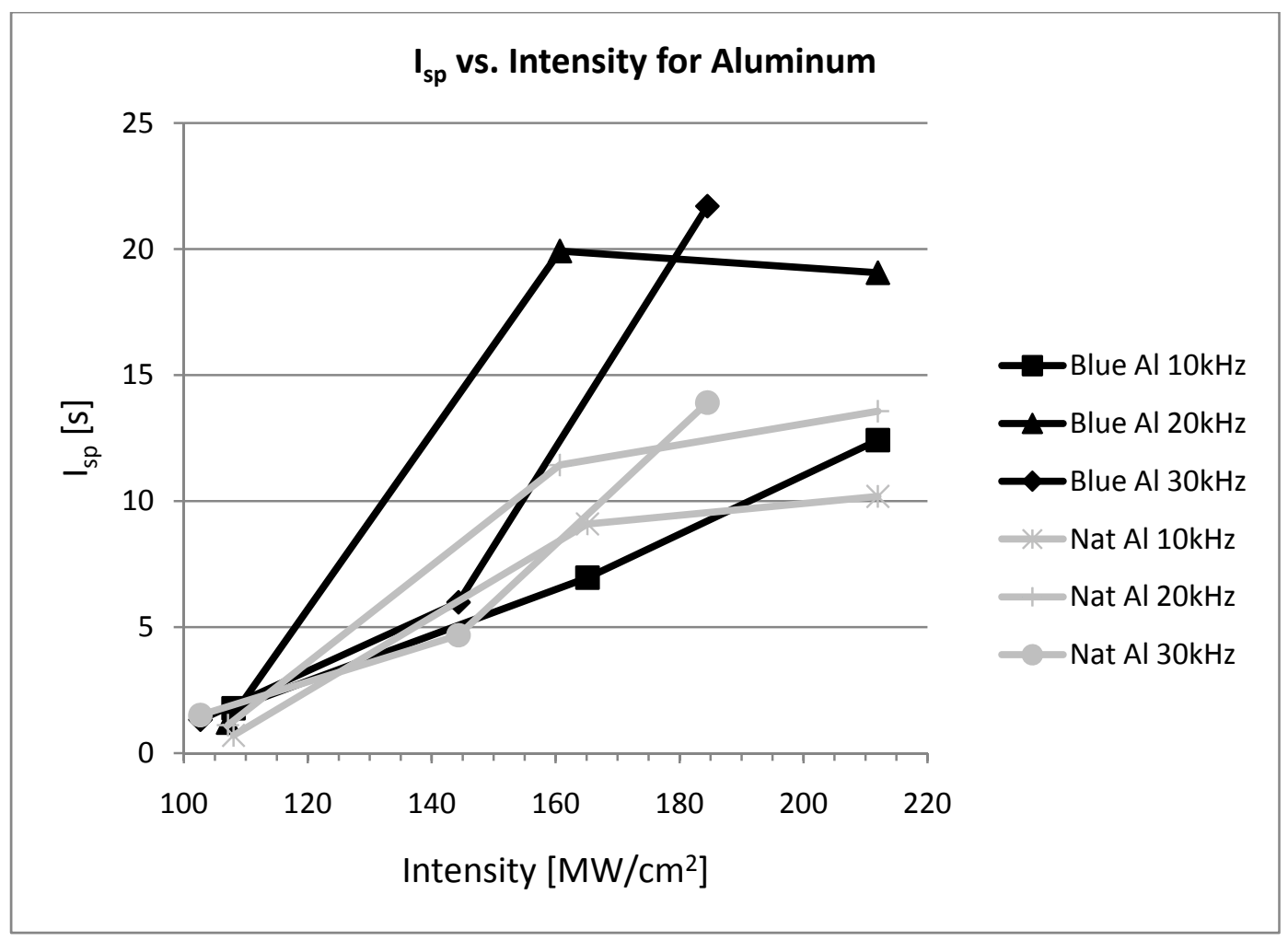

Figure 5-17: $I_{\text {sp }}$ vs. Intensity for Aluminum

Specific impulse results for aluminum are very reflective of the assumption previously made stating that the $I_{s p}$ for metals would be relatively high. Also, that the $C_{m}$ values are expected to be lower for the metals due to mass ablated per pulse; calculated to be approximately $0.75 \mathrm{ng} / \mathrm{pulse}$ for aluminum and $0.3 \mathrm{ng} /$ pulse for brass. These can be compared to $5 \mathrm{ng} /$ pulse for the thick PVC and approximately $1.8 \mu \mathrm{g} /$ pulse for the thin PVC samples, demonstrating the relation to mass ablated per pulse for $C_{m}$ and $I_{\text {sp. }}$. The reversed trends of the repetition rates are likely due to the ablation spot size being smaller for the higher repetition rates. The effects of the repetition rate with the material interaction and spot size are discussed in the Laser Material Interaction section of this chapter.

The specific impulse vs. laser intensity for PVC is shown below in Figure 5-18. 


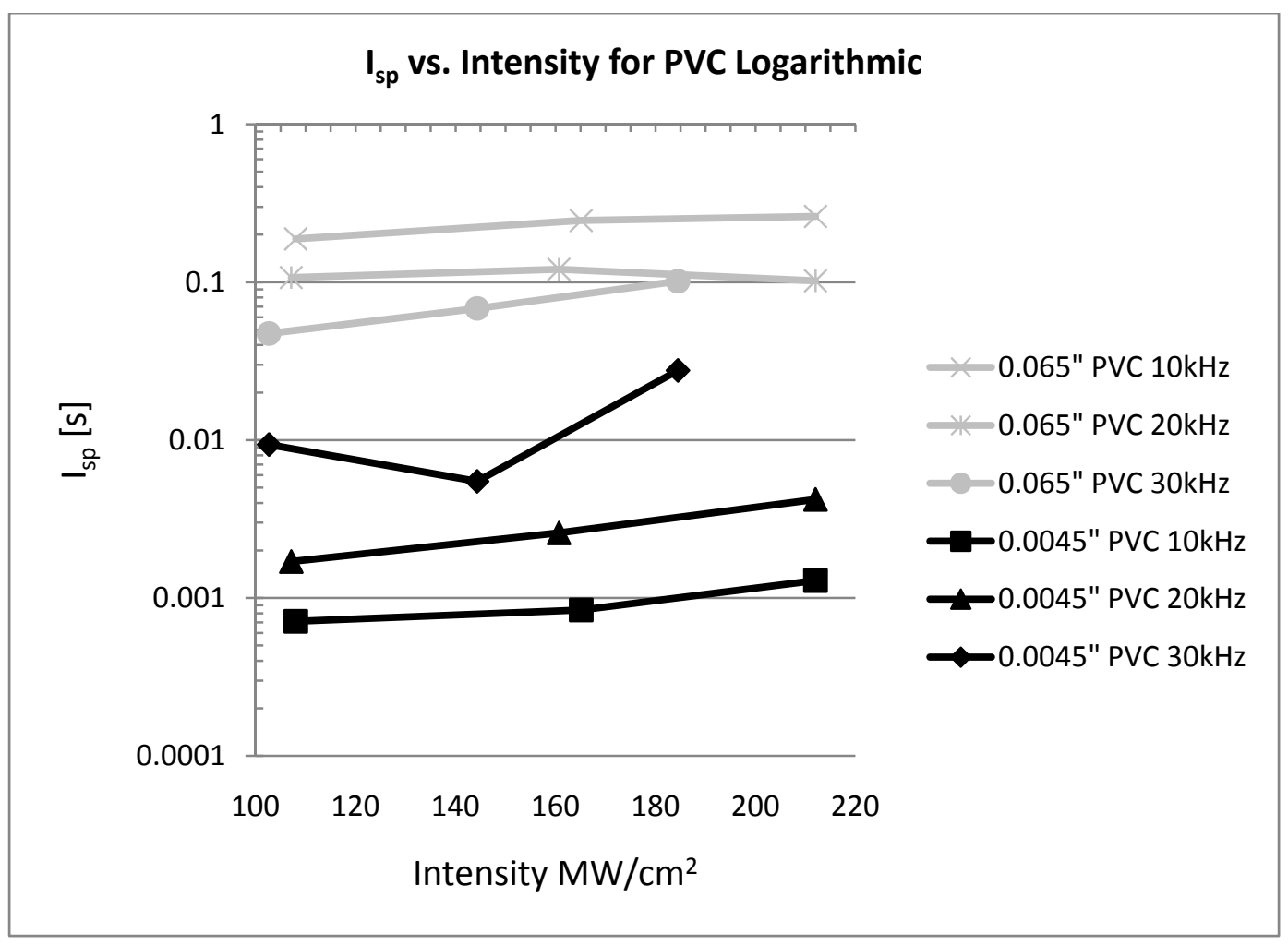

Figure 5-18: $I_{s p}$ vs. Intensity for PVC Logarithmic

The specific impulse results for PVC are very low due to the amount of ablated mass calculated per pulse. As previously mentioned, the ablated spot may actually be much larger than the spot contributing to the thrust. Polymers typically do not have high $I_{s p}$ values due to the amount of mass ablated per pulse, which explains why metals typically have higher $I_{s p}$ and lower $C_{m}$ values.

The specific impulse vs. laser intensity for brass is shown below in Figure 5-19. 


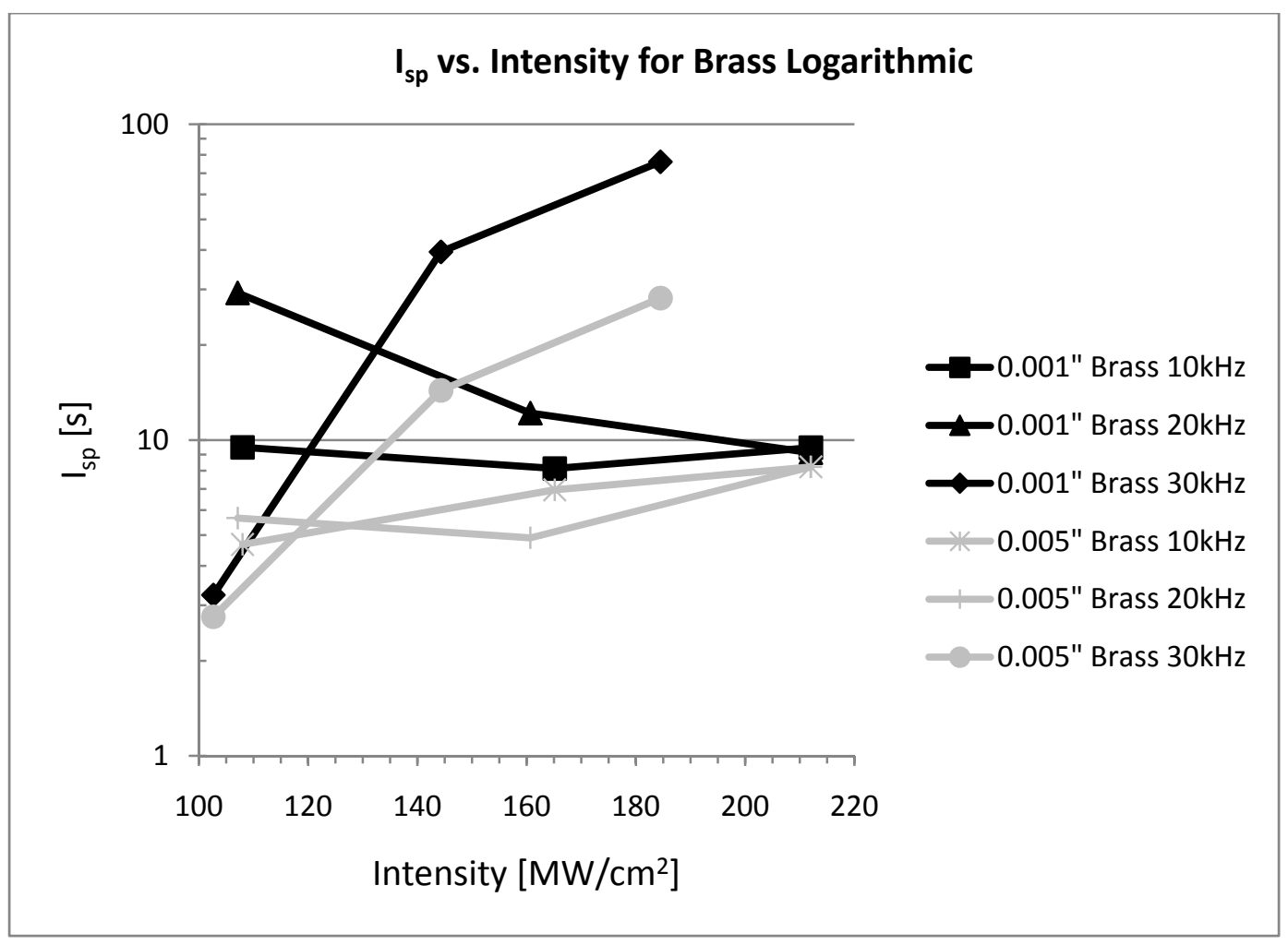

Figure 5-19: $I_{\text {sp }}$ vs. Intensity for Brass Logarithmic

Brass shows on average much higher $\mathrm{I}_{\mathrm{sp}}$ values than the other materials. The amount of mass ablated was calculated to be very low as brass ablated very quickly without a large HAZ. The highest point at 76s for $30 \mathrm{kHz}$ is thought to be unrealistic for this laser, and could be erroneous due to the ablation spot area calculation method used. The $0.001 " 20 \mathrm{kHz}$ data for the lowest pulse energy is also very high, most likely due to the same ablation area calculation method. The values would be assumed to remain relatively high if the ablation area was corrected. The ablation spot calculations were done via SEM images using the scale provided on the image. The samples viewed with SEM were only ablated for $0.3 \mathrm{~s}$, so the actual ablation spot size may be much larger than calculated. This was consistent over all of the samples and the ablated area was at relatively the same state, with the exception of the PVC whose surface had already cratered. 
A table of the peak values for each material is given below in Table 5-4.

\begin{tabular}{|l|c|c|c|c|c|c|}
\hline Material & $\begin{array}{c}\text { Aluminum } \\
\text { Blue }\end{array}$ & $\begin{array}{c}\text { Aluminum } \\
\text { Natural }\end{array}$ & $\begin{array}{c}\text { PVC } \\
0.065^{\prime \prime}\end{array}$ & $\begin{array}{c}\text { PVC } \\
0.0045^{\prime \prime}\end{array}$ & $\begin{array}{c}\text { Brass } \\
0.005^{\prime \prime}\end{array}$ & $\begin{array}{c}\text { Brass } \\
0.001^{\prime \prime}\end{array}$ \\
\hline $\mathrm{C}_{\mathrm{m}}[\mu \mathrm{Ns} / \mathrm{J}]$ & $\begin{array}{c}0.13 \\
@ 30 \mathrm{kHz}\end{array}$ & $\begin{array}{c}0.15 \\
@ 10 \mathrm{kHz}\end{array}$ & $\begin{array}{c}0.05 \\
@ 10 \mathrm{khz}\end{array}$ & $\begin{array}{c}0.61 \\
@ 30 \mathrm{kHz}\end{array}$ & $\begin{array}{c}0.11 \text { @10 } \\
\& 20 \mathrm{kHz}\end{array}$ & $\begin{array}{c}0.22 \\
@ 30 \mathrm{khz}\end{array}$ \\
\hline $\mathrm{I}_{\mathrm{sp}}[\mathrm{s}]$ & 22 & 14 & 0.26 & 0.028 & 28 & 76 \\
\hline $\begin{array}{l}\text { Mass ablated per } \\
\text { pulse [ng/pulse] }\end{array}$ & 0.82 & 0.65 & 5 & 1842 & 0.3 & 0.34 \\
\hline
\end{tabular}

Table 5-4: Max $C_{m}$ and $I_{s p}$ for each Material

Moment coupling coefficient and exhaust velocity are noticed to produce inverse results for the PVC sample specifically. The higher $C_{m}$ value for the thin PVC sample resulted in a very low $I_{s p}$, while the very low $C_{m}$ value for the thicker PVC resulted in a higher $I_{s p}$ comparatively. It is also noticed how the metals had lower $C_{m}$ results, however the $I_{s p}$ values are unexpectedly high. This confirms the previous assumption that metals would have a higher $\mathrm{V}_{\mathrm{ex}}$ due to the small amount of mass ablated per shot. The same energy from the laser pulse is deposited into a smaller volume. This results in less, but higher energy matter being ejected from the surface, resulting in high $\mathrm{I}_{\mathrm{sp}}$ values.

\subsubsection{Laser Material Interaction}

\subsubsection{Heat Affected Zone}

Laser ablation effects on materials are strongly dependent on the laser parameters. Heating the surrounding area of the ablation spot is a common problem with laser ablation applications. As was mentioned previously, the effects are not as critical for propulsion purposes. However, if a consistent flow of material were desired for a propulsion system then the material may not be able to produce the thrust values predicted due to the heat affected zone. Scanning electron microscope (SEM) images demonstrate the heat affected zone, even with the short 20ns pulses used for ablation. Figure 5-20 demonstrates a PVC sample ablated at $10 \mathrm{kHz}$ and approximately $248 \mu \mathrm{J}$ energy per pulse. 


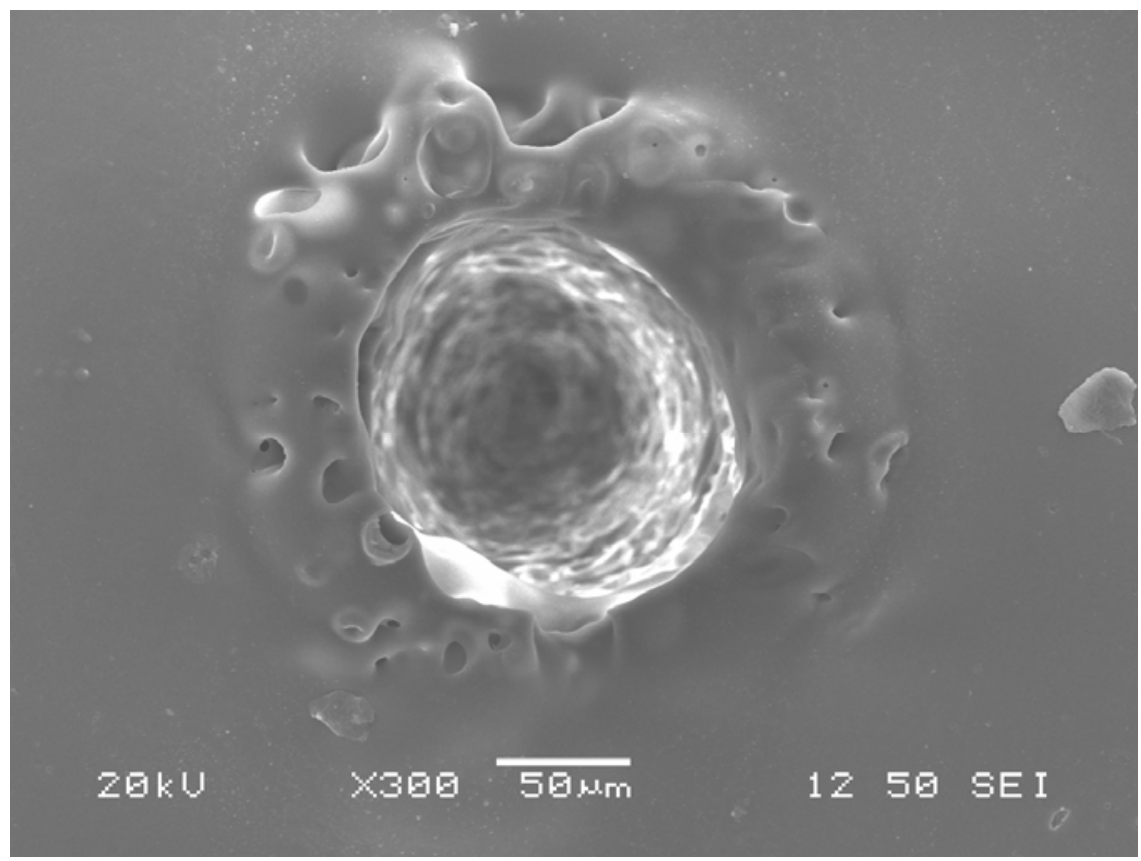

Figure 5-20: PVC at 10kHz, 248 $\mu$, HAZ

The zone directly surrounding the hole in Figure 5-20 is clearly a heat affected zone. This sample was ablated for approximately $0.3 \mathrm{~s}$. The ablation zone is much larger than the spot size, which leads to the assumption that the PVC was subjected to melting during ablation. The issue with the HAZ is for a sequential ablation system where the next ablation point would use the heat affected material for thrust production. It is assumed that with laser propulsion the entire propellant surface will be used for thrust production, but with a HAZ the actual impulse developed may actually be severely affected.

\subsubsection{Repetition Rate}

Repetition rate is considered very important for micromachining as the more frequent lower energy pulses aid in material removal while creating a lower HAZ. Figure 5-21 shows the progression from $10 \mathrm{kHz}$ to $40 \mathrm{kHz}$ of a thin 0.0065 " PVC sample. Each image is taken from a freshly ablated zone with the same elapsed time. 

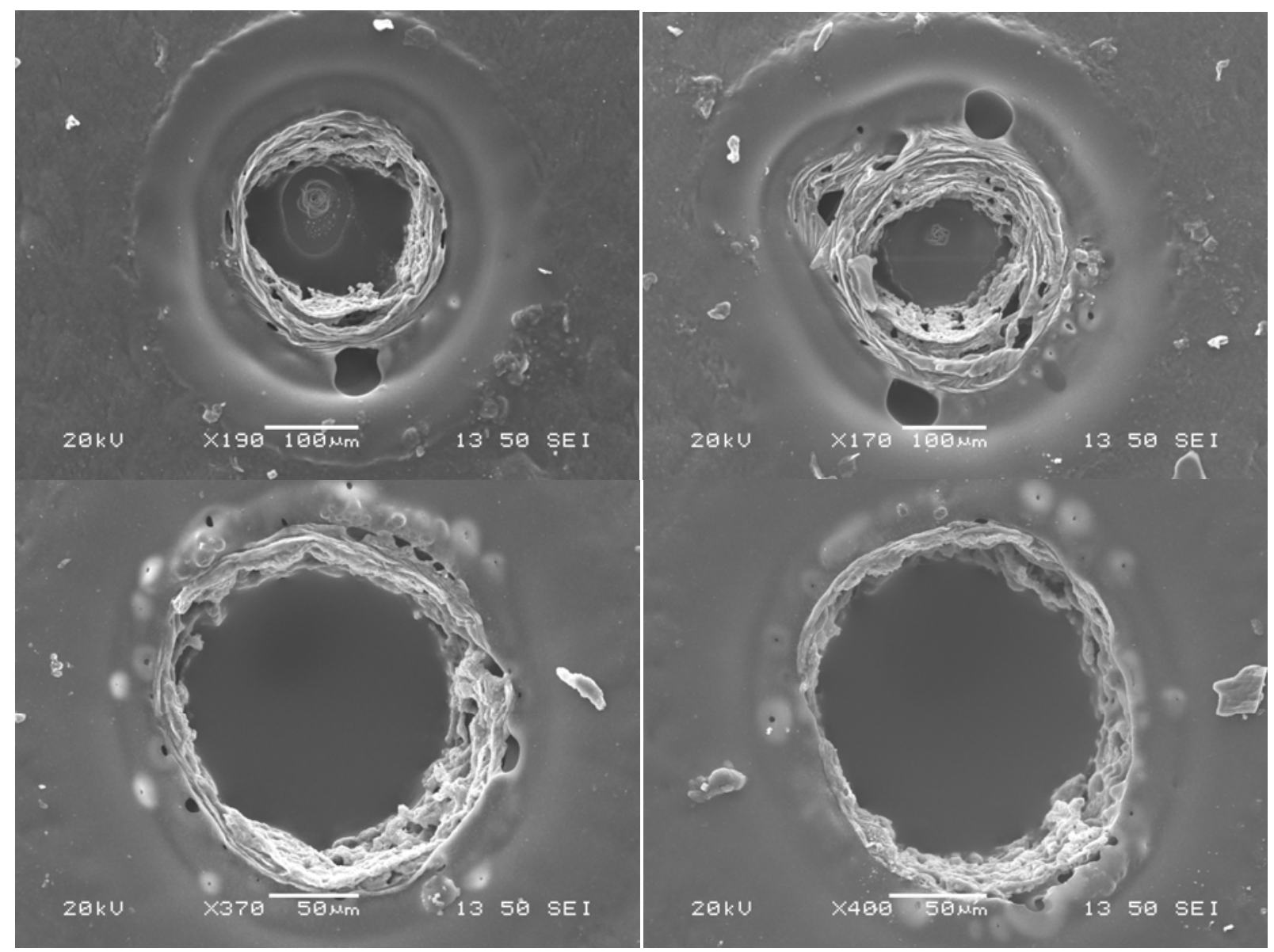

Figure 5-21: PVC (Top Left), 20kHz (Top Right), 30kHz (Bottom Left), and 40kHz (Bottom Right)

The progression shows the diameter of the HAZ reducing, along with the ablated area. The smaller ablation zone implies that a lower amount of material was ablated outside of the spot area. This is important to both manufacturing and propulsion as the amount of material lost will negatively reflect the laser ablation thruster's specific impulse and ablation efficiency. The top two images in Figure 5-21 also show an ablation of the glass plate holding the sample. The laser pulse intensity at $10 \mathrm{kHz}$ and $20 \mathrm{kHz}$ was high enough to pierce the material and then begin to ablate the glass behind it.

Another example of the effects of repetition rate on ablation is shown in Figure 5-22 below for brass. 

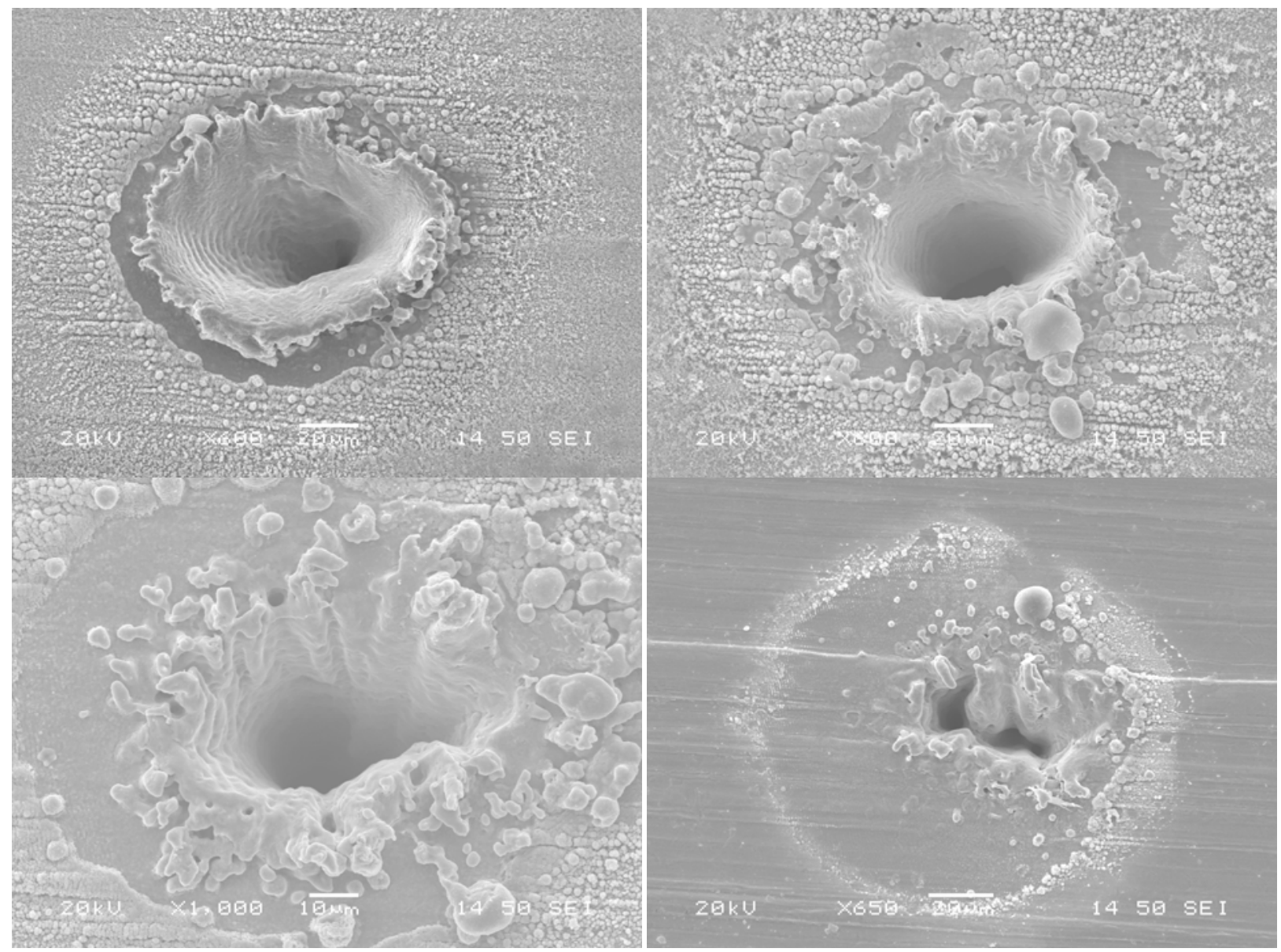

Figure 5-22: Brass at $10 \mathrm{kHz}$ (Top Left), $20 \mathrm{kHz}$ (Top Right), $30 \mathrm{kHz}$ (Bottom Left), and 40kHz (Bottom Right)

The laser spot seems to be creating a pear shaped ablation spot. The $40 \mathrm{kHz}$ demonstrates how the shape would have begun to form. This could be due to many factors including an issue with lens alignment. An assumption for this effect is the sample swinging during ablation where the sample is moving back and forth causing the laser to ablate another local spot, thus creating a pear shape. This ablation spot profile was not noticed in any of the other SEM images, so it is assumed that this profile is independent of the testing method and that this sample may not have been aligned properly.

\subsubsection{Dual Wavelength Spot Size Effects}

The dual wavelength effect essentially allows the material to absorb the laser light more efficiently. This is due to the material having a higher absorption coefficient at certain wavelength. Most of the materials will absorb the $532 \mathrm{~nm}$ wavelength to a higher degree than the $1064 \mathrm{~nm}$. The $1064 \mathrm{~nm}$ wavelength is then absorbed due to the electron density buildup from the initial lattice stimulation. The $532 \mathrm{~nm}$ wavelength radiation stimulates transitions of electrons from the bonding valence band to the anti-bonding conduction band, weakening the molecular bonding and increasing light absorption [69]. 
Figure 5-23 demonstrates the initial ablation spot size of a brass sample.

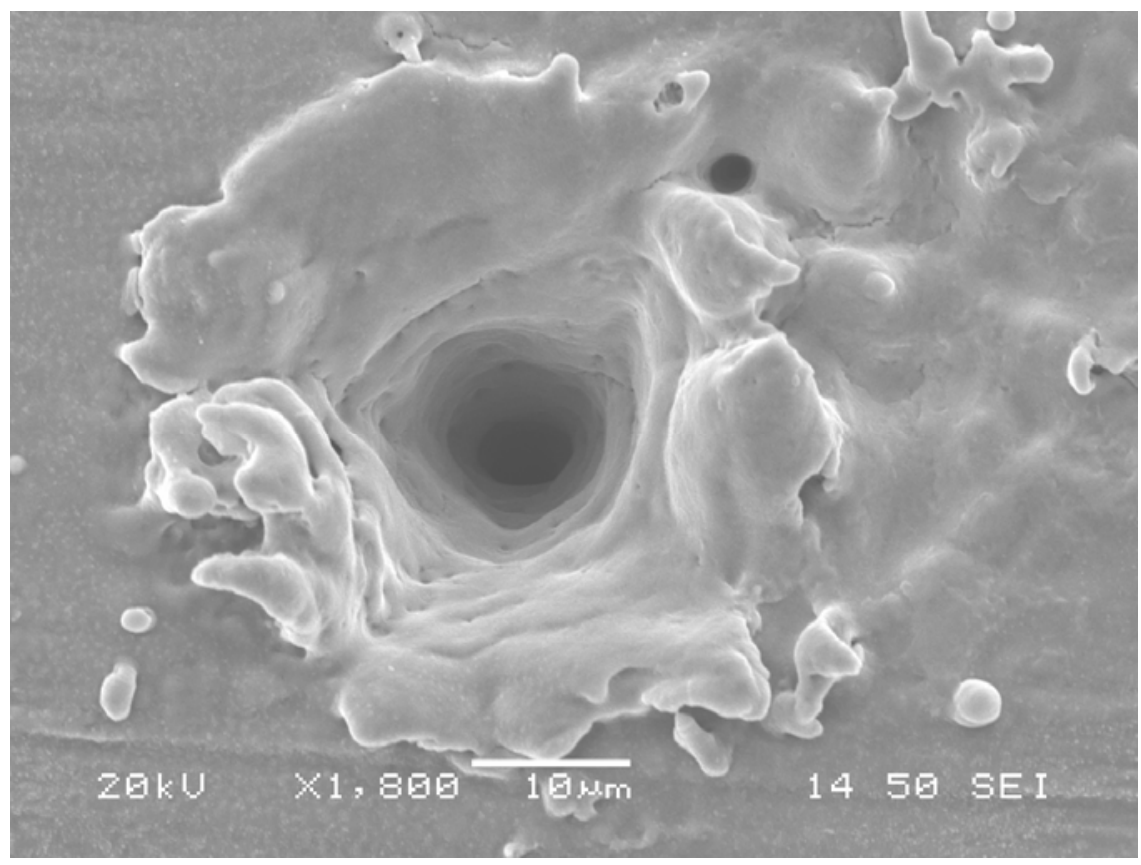

Figure 5-23: Brass $180 \mu \mathrm{J} 20 \mathrm{kHz}$ Spot Size

The ablation spot shown in this image is assumed to be an effect of the dual wavelength effect, from the $532 \mathrm{~nm}$ wavelength initially; $42.23 \mu \mathrm{m}$. The spot is gradually expanding with each pulse, and is expected to reach the spot diameter greater than the $1064 \mathrm{~nm}$ wavelength; $84.45 \mu \mathrm{m}$. This image also demonstrates melting encountered with laser ablation on certain materials. Brass was not expected to be affected by a HAZ as the PVC was. Brass also has a high degree of material redeposition due to atmospheric pressure, as shown in Figure 5-24. This would reduce the effectiveness of using a scale to measure material ablated, and further justifies using a volume to mass calculation method. 


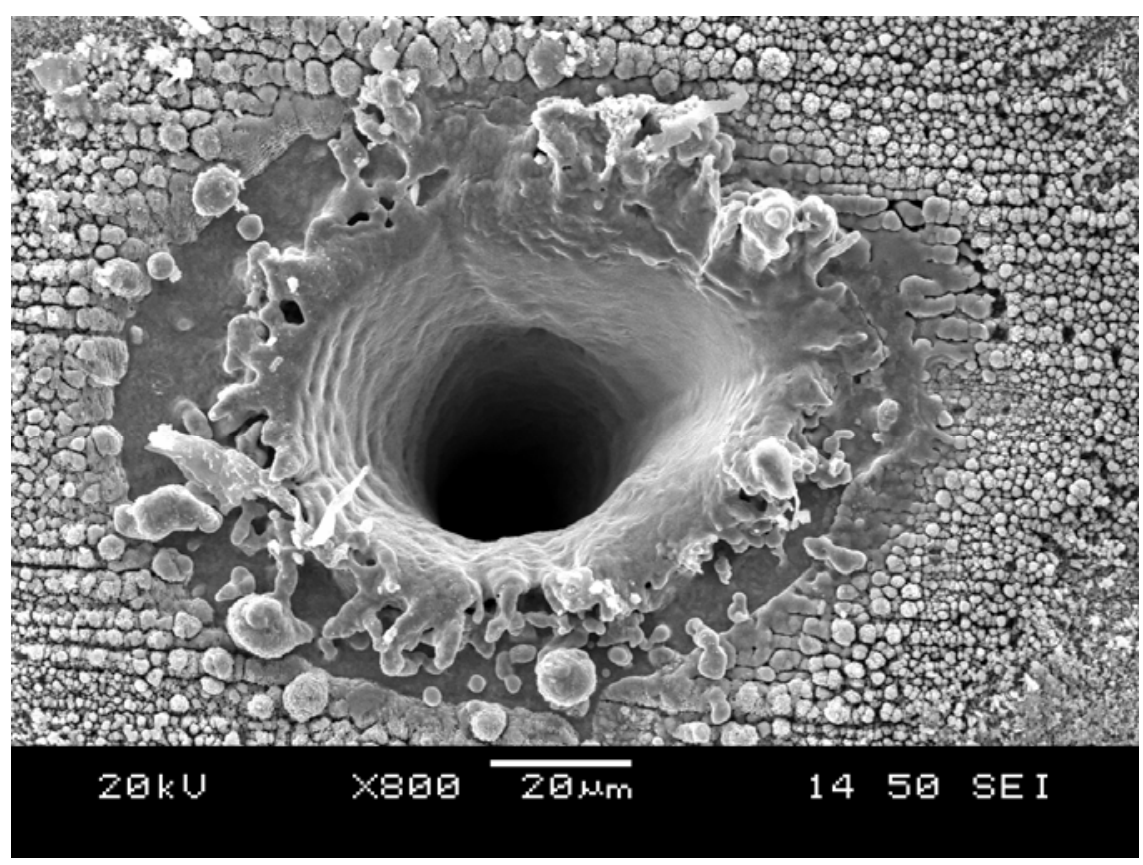

Figure 5-24: Brass $185 \mu \mathrm{J} 10 \mathrm{kHz}$ Redeposition

\subsection{Summary}

The results obtained in these tests compare well to those reviewed in the Experimental Review section of Chapter 1 of this thesis. The $C_{m}$ value is often used as a comparison of data. Pakhomov et al. found similar $C_{m}$ values to those calculated in this thesis. Pakhomov et al. used $8 \mathrm{~mJ}$ pulses to produce $20 \mu \mathrm{N} / \mathrm{W}$ to $80 \mu \mathrm{N} / \mathrm{W}$ moment coupling coefficients [18]. Aluminum in these tests produced $0.13 \mu \mathrm{N} / \mathrm{W}$ at $207 \mu \mathrm{J}$ energy per pulse. The natural aluminum and thick PVC had maximum $\mathrm{C}_{m}$ values at $10 \mathrm{kHz}$ which is assumed to result due to the reflective surface of the natural aluminum, and the PVC melting surrounding material. The pulse energies are not drastically different, so the 0.065 " PVC would produce more thrust at the higher pulse energy due to the HAZ not differing much. The higher $\mathrm{I}_{\text {sp }}$ results from the higher repetition rates are assumed to be a result of the lower amount of material ablated per pulse, and due to the HAZ not expanding the ablation spot as much as the lower repetition rates. This theory is expected to perform similarly on a larger scale. Higher repetition rates could provide enhanced results, however, the threshold for plume lifespan may be a limiting factor as pulse energy impact on the propellant may degrade during constant plasma interaction. The plume could essentially be slower than the repetition rate. The effects of such a behavior are unknown, and could be investigated in future testing. 


\section{Chapter 6: Summary and Future Work}

\subsection{Summary}

Greater interest in energy conservation has recently spiked an increase in technologies that reduce power consumption and reduce energy conversion losses for propulsion systems. Laser propulsion has the benefits of being able to have an independent power source off ship and provide energy to the propulsion system onboard. Conversely, diode lasers can be manufactured at very small dimensions relative to other rocket systems to be used as an on-board power source. The efficiency of the propellant usage is also a benefit to the laser propulsion system. Laser propulsion is currently in its preliminary experimentation phase. Many factors that contribute to the ablative propulsion phenomenon are still unknown. This research contributed to the knowledge of laser interactions with propellant to produce propulsive forces. To the knowledge of the author, using a nanosecond pulsed high repetition rate laser was not previously evaluated for thrust production. This thesis evaluated the effects of the thrust production with several high repetition rates, and also further evaluated the visible propellant damage as a result of laser ablation.

In this research, an apparatus based on a direct force measurement method was developed. Using direct force measurement, a cost effective method to attain comparative data was developed. The ultimate goal of this design was to compare the effects of differing laser parameters to the resulting thrust output. Further calibration is required for future data accuracy and validation. However, the system responded very well to the high repetition rate laser and provided consistent results. Very little setup and very few devices were required, which further reduced complexity and cost of the system.

The results obtained in this thesis demonstrated the effects of a high repetition rate laser at $10 \mathrm{kHz}$, $20 \mathrm{kHz}$, and $30 \mathrm{kHz}$ used for propulsion. A decreasing thrust was expected as the repetition rate increased, but the pulse energy proved to be the dominant parameter with relation to thrust and $\mathrm{C}_{\mathrm{m}}$. The $C_{m}$ and $I_{s p}$ were found to be much higher for the metals as expected, due to the ablated mass per pulse. The trends with repetition rate for the data compiled reversed for the $I_{s p}$ values. For thrust and $\mathrm{C}_{m}$, the trend related their values to the inverse of the repetition rate due to decreasing pulse energies. However, for $I_{s p}$ the trend was reversed and the higher repetition rates resulted in higher $I_{s p}$ values due to the reduced mass ablated. This is an important point to note as $I_{s p}$ is regarded as a very important value with respect to laser propulsion. 
A higher repetition rate laser could effectively be used to increase the $I_{s p}$. A higher power laser would be required to produce any useable thrust levels as the values obtained in this thesis are considered too low for satellite maneuvering. By using a laser and propellant that could provide the thrust necessary, an increase in the repetition rate of the laser could further increase the usable propellant amount and in turn increase the $I_{s p}$ and total impulse of the system. The repetition rate is believed to be regulated by the plasma plume expansion time. A repetition rate above $500 \mathrm{kHz}$ is not recommended for laser propulsion as the plasma-pulse interaction may degrade the pulse energy. This theory could be applied to laser micropropulsion, and is expected to produce similar results on a larger scale for other applications.

\subsection{Future Work}

Calibration is essential for this system to be used any further. The data seems to compare well with published data and with expected trends. The resonance of the system is also very important to discover as the system could have been severely affected by system resonance. A calibrated noise cancelling amplifier is suggested for use with these extremely sensitive piezoelectric films as the signals received are difficult to predict and filter. Further testing using this system would require a more rigid testing apparatus that could improve the consistency of the sample placement with respect to the laser beam. If at all possible, a lower repetition rate is suggested for future testing with this system for data comparison. Higher repetition rates should also be used to evaluate the plasma-pulse interaction effects. The effects of testing in a vacuum chamber could also serve as a data comparison method, and could evaluate the differences in using the high repetition rate at atmospheric pressure vs. a vacuum.

If data comparison or calibration is not effective or available, it is suggested to purchase a readily available direct measurement device for future tests. The issues that arose using this testing method were very educational but also difficult as there was very little information available regarding the behavior of PVDF films for similar low load applications. 


\section{Appendix A: MATLAB Code and Simulink Filters}

\section{Calibration}

\% Load and Run Oscilloscope Data for Calibration

\%Clear relevant workspace data

clear xYTcal xYTfiltcal

$\%$ Load data from Workspace

$x=\operatorname{CALIBDATA}(:, 1)+0.4$;

$y=\operatorname{CALIBDATA}(:, 2)$;

$y u=(y+0.085) ; \quad$ \%Centering signal from offset

$\%$ Calculate Thrust

YT = yu/1000; $\quad$ \%Convert signal from amplified to raw data

$\mathrm{xYTcal}=[\mathrm{X}, \mathrm{YT}] ; \quad$ \%creating an array from time and force

\%\% Thrust Plot - plot original signal from oscilloscope

figure('name', 'Voltage', 'numbertitle', 'off')

$\operatorname{plot}\left(\mathrm{x}, \mathrm{YT}, \mathrm{I}^{\prime}\right)$, grid on

xlabel('Time, [s]')

ylabel('Voltage pre amp, [V]')

title('Voltage from dropped mass')

\%\% Run filter made with Simulink by sending XYT

set_param('calib_filter', 'SimulationCommand', 'start')

pause (5); $\quad \%$ Waiting for filter to stop running

$\%$ Plot filtered signal

figure('name', 'Filtered Calibration Signal', 'numbertitle', 'off')

plot (xYTfiltcal)

xlabel('Time, [s]')

ylabel('Filtered Voltage Pre Amplification, [V]')

title('Filtered Voltage from dropped mass')

\% Calibration

mass $=63 \mathrm{e}-9 ; \quad$ \%Defining the mass in $\mathrm{kg}$

$y=0.00295 ; \quad$ \%Vertical drop height

$\mathrm{a}=9.81 ; \quad$ \%Acceleration due to gravity

$v=\operatorname{sqrt}\left(2^{*} a^{*} y\right) ; \quad$ \%Velocity at impact

\%Impact Force

Ftest $=\max ($ abs $(x Y T f i l t c a l)) \quad$ Maximum force from filtered test data

Fcalcmv $=$ mass $^{*} \mathrm{v} / 0.001 \quad$ \%Calculated Impact force 


\section{Calibration Filter}

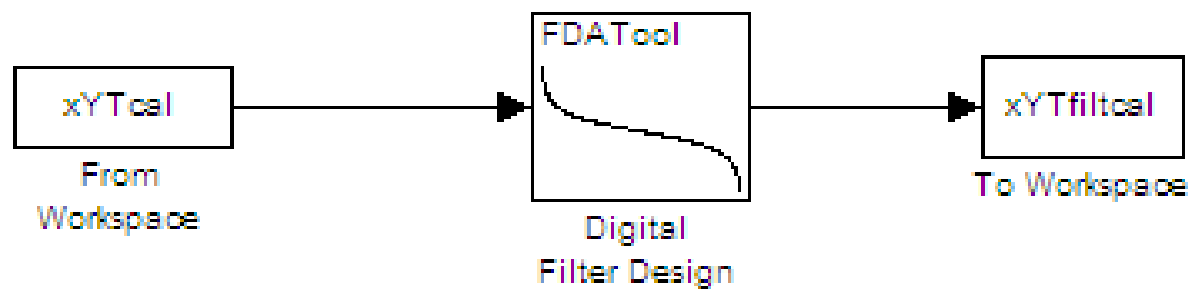

The filter is a low pass filter, set up as follows:

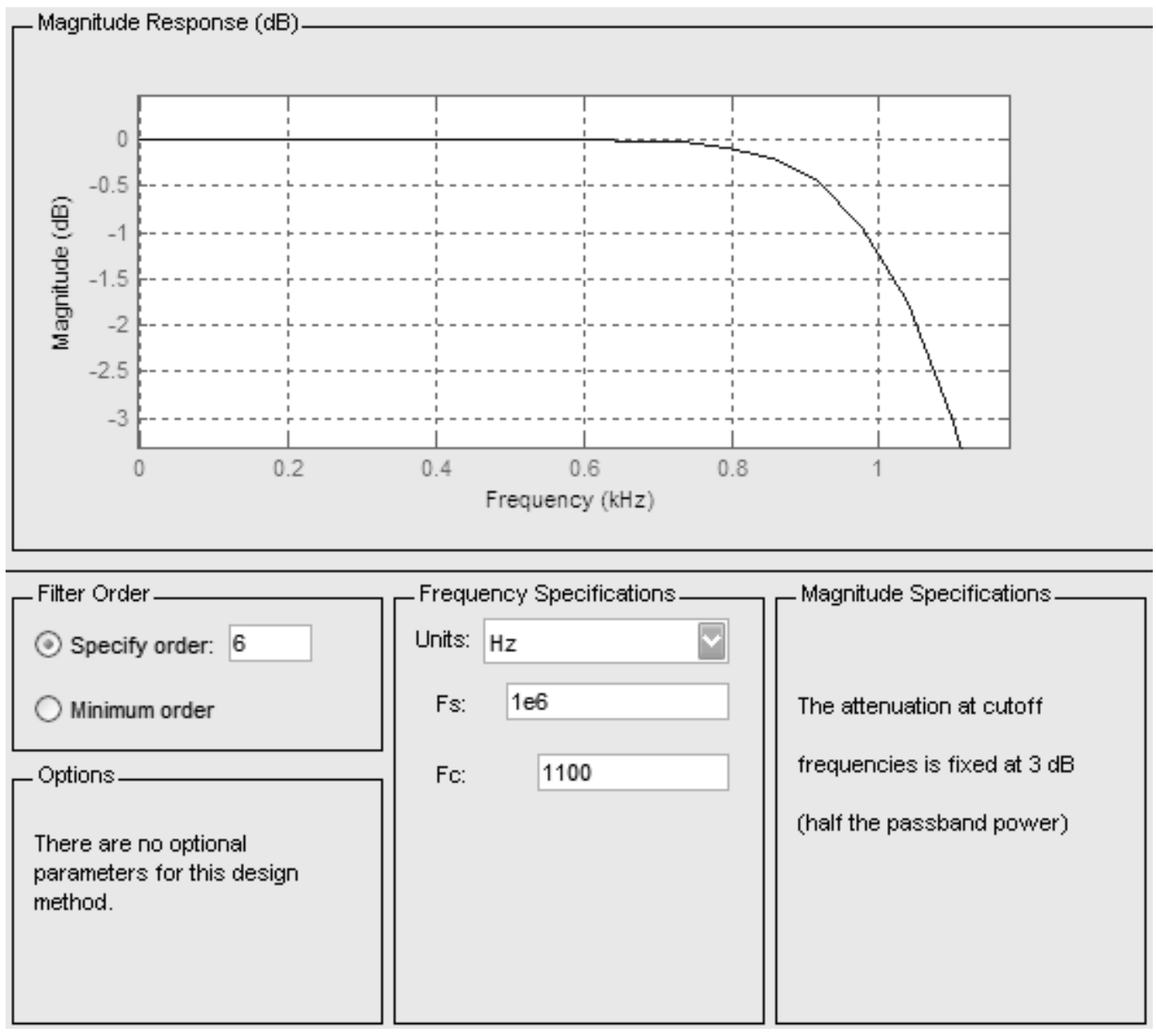




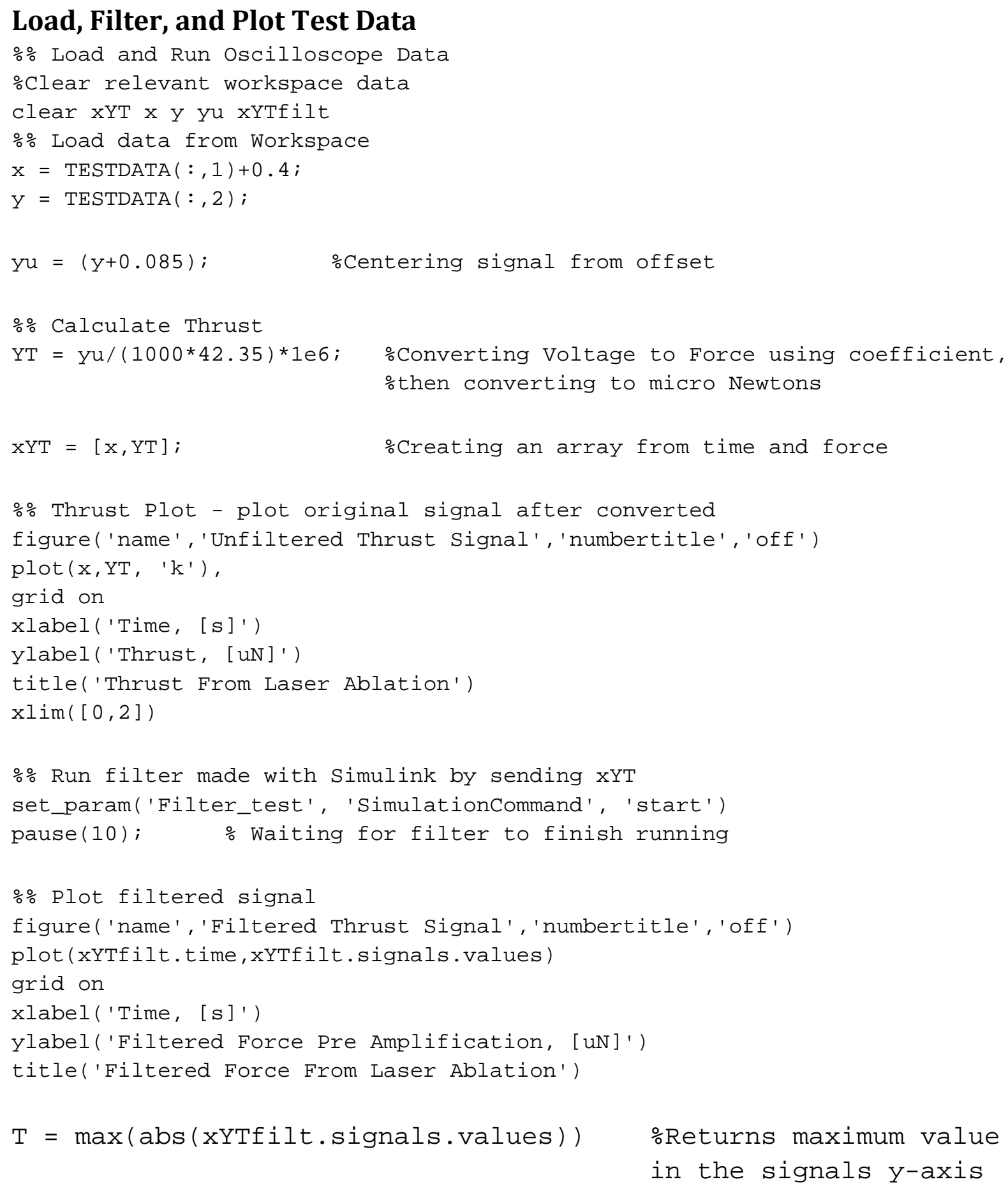




\section{Test Filter}

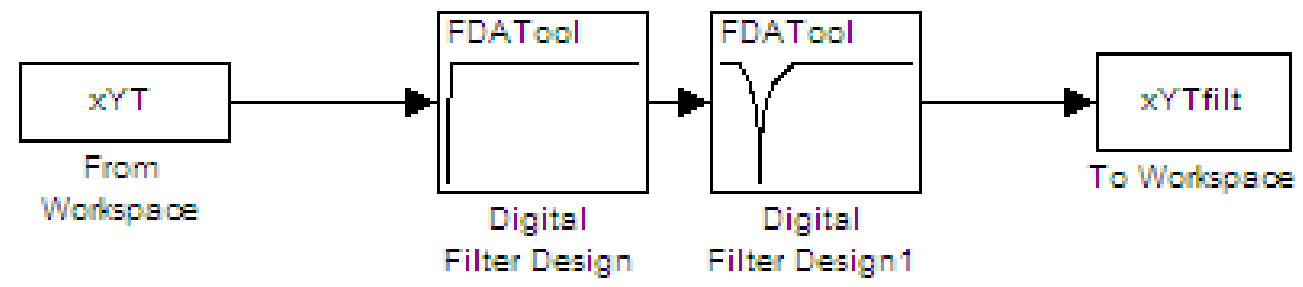

Where the first filter is a High Pass filter, set up as follows:

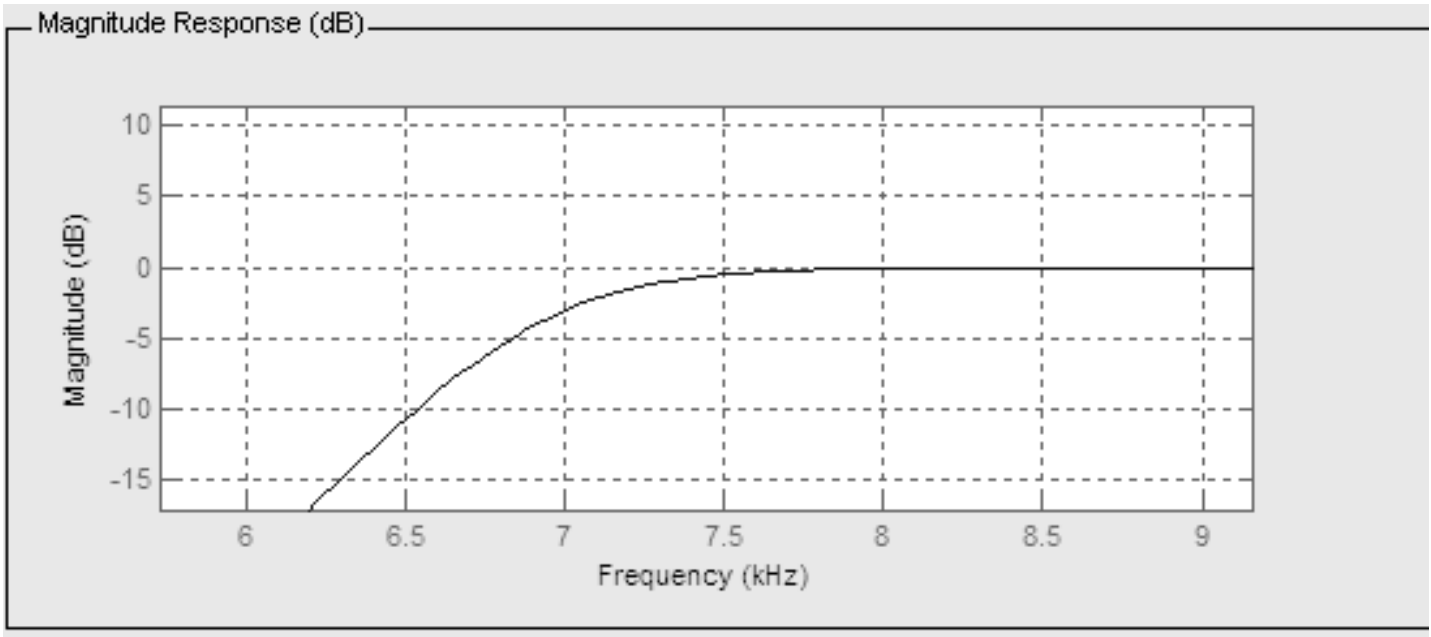

\begin{tabular}{|c|c|c|c|}
\hline — Filter Order & - Frequ & ency Specifications & - Magnitude Specifications_- \\
\hline (-) Specify order: 16 & Units: & $\mathrm{Hz}$ & \\
\hline Minimum order & Fs: & $1 \mathrm{e} 6$ & The attenuation at cutoff \\
\hline $\begin{array}{l}\text { Options } \\
\text { There are no optional } \\
\text { parameters for this design } \\
\text { method. }\end{array}$ & $\mathrm{Fc}:$ & 7000 & $\begin{array}{l}\text { frequencies is fixed at } 3 \mathrm{~dB} \\
\text { (half the passband power) }\end{array}$ \\
\hline
\end{tabular}


The second filter is a notch type filter, set up as follows:

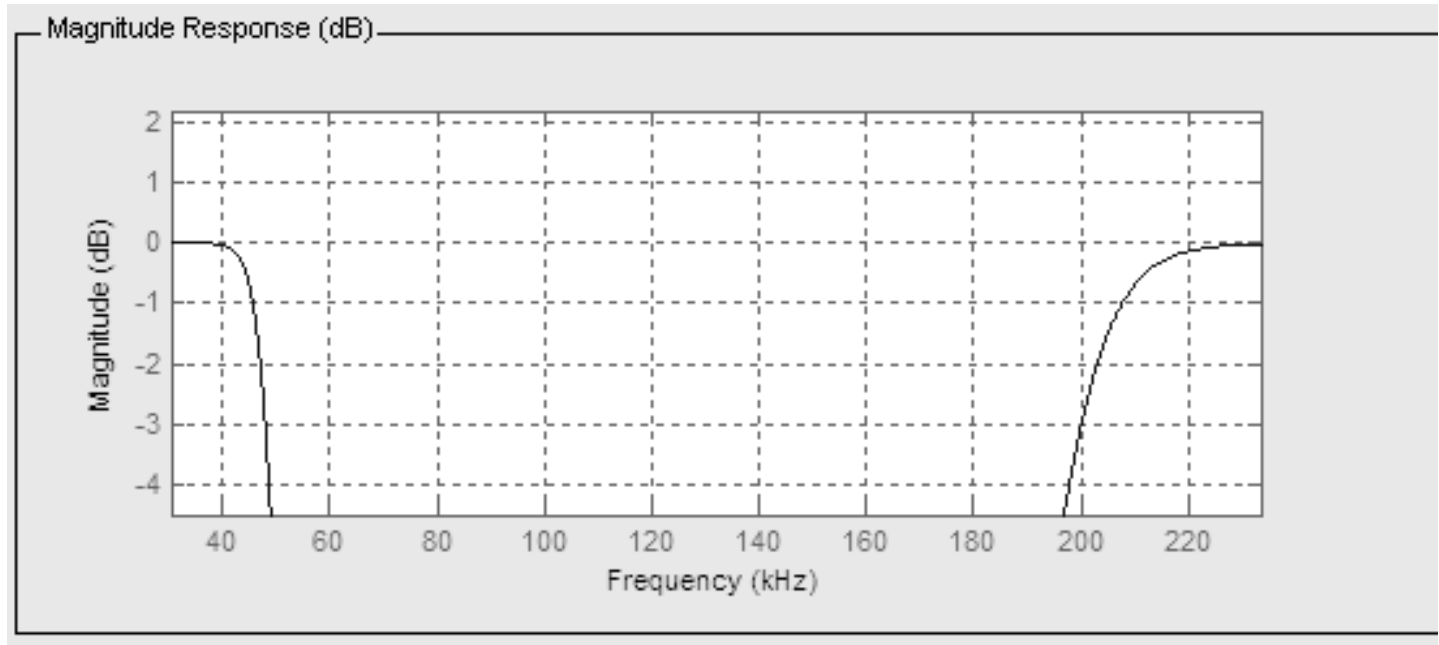

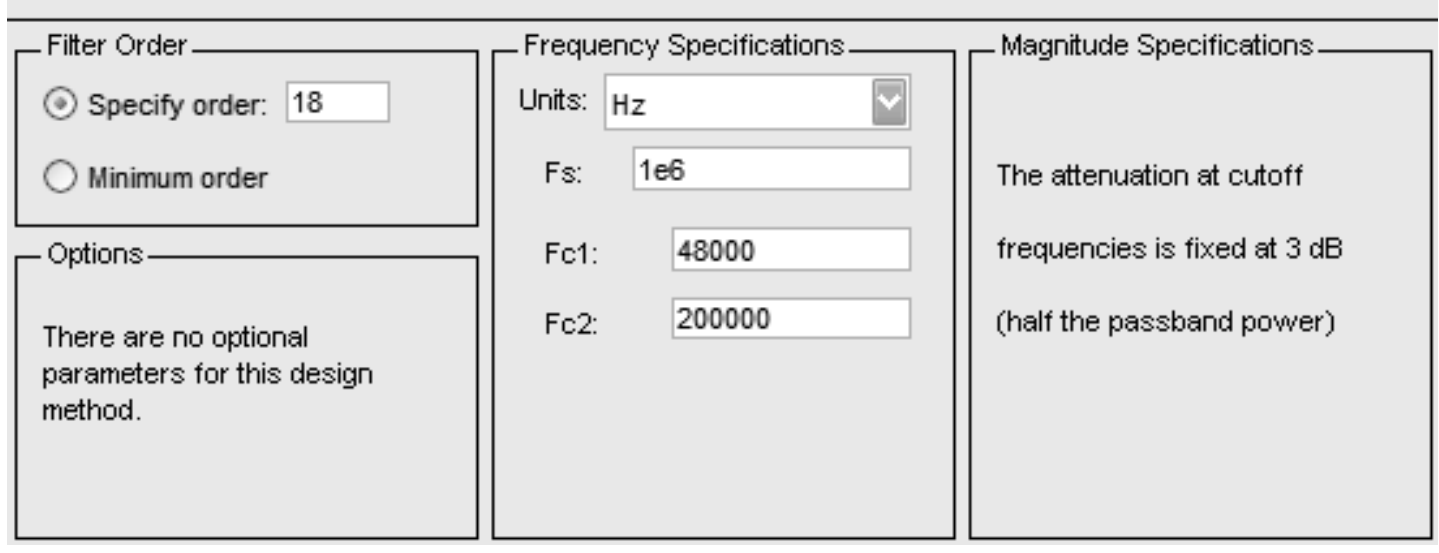




\section{Appendix B: Amplifier Schematics}

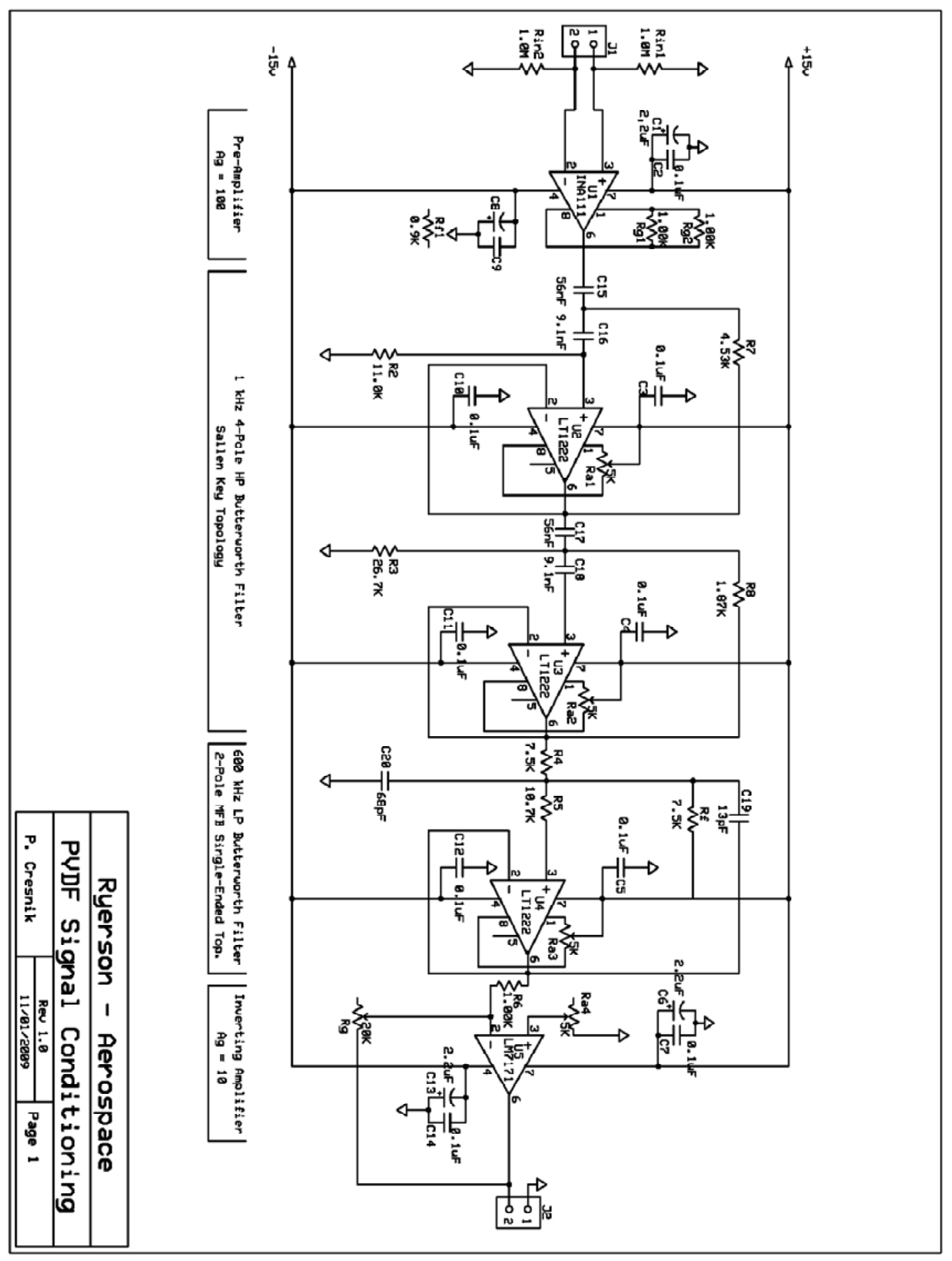




\section{Appendix C: Test Data Compilation}

\begin{tabular}{|c|c|c|c|c|c|c|c|c|}
\hline Sample & $\begin{array}{c}\text { Pulse } \\
\text { Energy } \\
{[\mu \mathrm{J} / \text { pulse] }}\end{array}$ & $\begin{array}{l}\text { Repetition } \\
\text { Rate }[\mathrm{kHz}]\end{array}$ & $\begin{array}{l}\text { Intensity } \\
{\left[\mathrm{W} / \mathrm{cm}^{2}\right]}\end{array}$ & $\begin{array}{l}\text { Thrust } \\
{[\mu \mathrm{N}]}\end{array}$ & $\begin{array}{l}\text { Standard } \\
\text { Deviation }\end{array}$ & $\begin{array}{l}\text { Mass Ablated } \\
\text { per pulse } \\
\text { [ng/pulse] }\end{array}$ & $\begin{array}{c}\mathrm{Cm} \\
{[\mu \mathrm{Ns} / \mathrm{J}]}\end{array}$ & Isp [s] \\
\hline $\begin{array}{l}\text { Blue } \\
\text { Aluminum }\end{array}$ & 121 & 10 & 108 & 13.3 & 2.18 & 0.57 & 0.083 & 1.783 \\
\hline $\begin{array}{l}\text { Blue } \\
\text { Aluminum }\end{array}$ & 185 & 10 & 165 & 29.1 & 2.97 & 0.32 & 0.118 & 6.967 \\
\hline $\begin{array}{l}\text { Blue } \\
\text { Aluminum }\end{array}$ & 237.5 & 10 & 212 & 40.5 & 0.26 & 0.25 & 0.128 & 12.422 \\
\hline $\begin{array}{l}\text { Blue } \\
\text { Aluminum }\end{array}$ & 120 & 20 & 107 & 8.7 & 3.32 & 0.54 & 0.054 & 1.218 \\
\hline $\begin{array}{l}\text { Blue } \\
\text { Aluminum }\end{array}$ & 180 & 20 & 160 & 27.0 & 1.77 & 0.10 & 0.112 & 19.923 \\
\hline $\begin{array}{l}\text { Blue } \\
\text { Aluminum }\end{array}$ & 237.5 & 20 & 212 & 37.7 & 3.75 & 0.15 & 0.119 & 19.063 \\
\hline $\begin{array}{l}\text { Blue } \\
\text { Aluminum }\end{array}$ & 115 & 30 & 102 & 6.0 & N/A & 0.35 & 0.039 & 1.327 \\
\hline $\begin{array}{l}\text { Blue } \\
\text { Aluminum }\end{array}$ & 162 & 30 & 144 & 13.0 & 0.28 & 0.17 & 0.060 & 5.986 \\
\hline $\begin{array}{l}\text { Blue } \\
\text { Aluminum }\end{array}$ & 207 & 30 & 184 & 36.7 & 4.95 & 0.13 & 0.133 & 21.705 \\
\hline $\begin{array}{l}\text { Natural } \\
\text { Aluminum }\end{array}$ & 121 & 10 & 108 & 5.3 & 0.04 & 0.57 & 0.033 & 0.706 \\
\hline $\begin{array}{l}\text { Natural } \\
\text { Aluminum }\end{array}$ & 185 & 10 & 165 & 38.0 & 3.68 & 0.32 & 0.154 & 9.097 \\
\hline $\begin{array}{l}\text { Natural } \\
\text { Aluminum }\end{array}$ & 237.5 & 10 & 212 & 33.2 & 3.96 & 0.25 & 0.105 & 10.191 \\
\hline $\begin{array}{l}\text { Natural } \\
\text { Aluminum }\end{array}$ & 120 & 20 & 107 & 6.0 & N/A & 0.41 & 0.037 & 1.117 \\
\hline $\begin{array}{l}\text { Natural } \\
\text { Aluminum }\end{array}$ & 180 & 20 & 160 & 11.6 & N/A & 0.08 & 0.048 & 11.434 \\
\hline $\begin{array}{l}\text { Natural } \\
\text { Aluminum }\end{array}$ & 237.5 & 20 & 212 & 20.1 & 7.92 & 0.11 & 0.063 & 13.570 \\
\hline $\begin{array}{l}\text { Natural } \\
\text { Aluminum }\end{array}$ & 115 & 30 & 102 & 5.7 & N/A & 0.29 & 0.037 & 1.513 \\
\hline $\begin{array}{l}\text { Natural } \\
\text { Aluminum }\end{array}$ & 162 & 30 & 144 & 8.5 & 0.18 & 0.14 & 0.039 & 4.683 \\
\hline $\begin{array}{l}\text { Natural } \\
\text { Aluminum }\end{array}$ & 207 & 30 & 184 & 19.6 & 4.04 & 0.11 & 0.071 & 13.910 \\
\hline $0.065 "$ PVC & 121 & 10 & 108 & 4.2 & 0.68 & 1.72 & 0.026 & 0.188 \\
\hline $0.065 "$ PVC & 185 & 10 & 165 & 10.1 & 4.07 & 3.15 & 0.041 & 0.246 \\
\hline $0.065 "$ PVC & 237.5 & 10 & 212 & 17.0 & 0.90 & 4.98 & 0.054 & 0.261 \\
\hline $0.065 "$ PVC & 120 & 20 & 107 & 3.1 & 1.05 & 2.22 & 0.019 & 0.107 \\
\hline
\end{tabular}




\begin{tabular}{|c|c|c|c|c|c|c|c|c|}
\hline $0.065^{\prime \prime}$ PVC & 180 & 20 & 160 & 4.6 & 0.67 & 2.89 & 0.019 & 0.121 \\
\hline $0.065 "$ PVC & 237.5 & 20 & 212 & 4.0 & 0.31 & 3.03 & 0.013 & 0.102 \\
\hline $0.065 "$ PVC & 115 & 30 & 102 & 1.6 & 0.07 & 2.50 & 0.010 & 0.047 \\
\hline $0.065 "$ PVC & 162 & 30 & 144 & 2.0 & 0.28 & 2.28 & 0.009 & 0.068 \\
\hline $0.065 "$ PVC & 207 & 30 & 184 & 3.1 & 0.75 & 2.32 & 0.011 & 0.102 \\
\hline $0.0045 "$ PVC & 121 & 10 & 108 & 13.5 & 1.41 & 1450.85 & 0.084 & 0.001 \\
\hline $0.0045 "$ PVC & 185 & 10 & 165 & 15.9 & 8.76 & 1450.85 & 0.065 & 0.001 \\
\hline $0.0045 "$ PVC & 237.5 & 10 & 212 & 24.5 & 5.02 & 1450.85 & 0.077 & 0.001 \\
\hline $0.0045 "$ PVC & 120 & 20 & 107 & 15.1 & 7.81 & 675.98 & 0.094 & 0.002 \\
\hline $0.0045 "$ PVC & 180 & 20 & 160 & 22.8 & 6.26 & 675.98 & 0.095 & 0.003 \\
\hline $0.0045 "$ PVC & 237.5 & 20 & 212 & 37.3 & 15.20 & 675.98 & 0.118 & 0.004 \\
\hline 0.0045" PVC & 115 & 30 & 102 & 45.1 & 25.63 & 368.44 & 0.294 & 0.009 \\
\hline $0.0045 "$ PVC & 162 & 30 & 144 & 131.8 & 17.61 & 1842.18 & 0.612 & 0.005 \\
\hline $0.0045 "$ PVC & 207 & 30 & 184 & 133.0 & 32.53 & 368.44 & 0.483 & 0.028 \\
\hline 0.001" Brass & 121 & 10 & 108 & 14.1 & 11.24 & 0.11 & 0.088 & 9.481 \\
\hline 0.001" Brass & 185 & 10 & 165 & 25.7 & 1.73 & 0.24 & 0.104 & 8.126 \\
\hline 0.001" Brass & 237.5 & 10 & 212 & 32.7 & 6.22 & 0.26 & 0.103 & 9.458 \\
\hline 0.001" Brass & 120 & 20 & 107 & 18.6 & 5.06 & 0.05 & 0.116 & 29.255 \\
\hline 0.001" Brass & 180 & 20 & 160 & 36.3 & 12.37 & 0.23 & 0.151 & 12.178 \\
\hline 0.001" Brass & 237.5 & 20 & 212 & 31.5 & 0.71 & 0.26 & 0.099 & 9.125 \\
\hline 0.001" Brass & 115 & 30 & 102 & 12.4 & 2.69 & 0.29 & 0.081 & 3.233 \\
\hline 0.001" Brass & 162 & 30 & 144 & 32.6 & 7.12 & 0.06 & 0.151 & 39.448 \\
\hline 0.001" Brass & 207 & 30 & 184 & 61.0 & 16.35 & 0.06 & 0.221 & 76.099 \\
\hline 0.005" Brass & 121 & 10 & 108 & 12.0 & 0.00 & 0.20 & 0.074 & 4.673 \\
\hline 0.005" Brass & 185 & 10 & 165 & 27.3 & 6.51 & 0.30 & 0.111 & 6.956 \\
\hline 0.005" Brass & 237.5 & 10 & 212 & 35.5 & 2.12 & 0.33 & 0.112 & 8.227 \\
\hline 0.005" Brass & 120 & 20 & 107 & 13.0 & 1.80 & 0.18 & 0.081 & 5.665 \\
\hline 0.005" Brass & 180 & 20 & 160 & 18.3 & 0.21 & 0.28 & 0.076 & 4.905 \\
\hline 0.005" Brass & 237.5 & 20 & 212 & 35.5 & 2.12 & 0.33 & 0.112 & 8.227 \\
\hline
\end{tabular}




\begin{tabular}{|c|c|c|c|c|c|c|c|c|}
\hline 0.005 " Brass & 115 & 30 & 102 & 8.5 & N/A & 0.34 & 0.080 & 2.757 \\
\hline $0.005 "$ Brass & 162 & 30 & 144 & 13.7 & 3.11 & 0.07 & 0.064 & 14.360 \\
\hline $0.005 "$ Brass & 207 & 30 & 184 & 22.9 & 0.53 & 0.06 & 0.083 & 28.140 \\
\hline & & & \multicolumn{2}{|c|}{ Average Deviation } & 5.06 & & & \\
\hline
\end{tabular}

*N/A standard deviations are tests with limited data 


\section{Bibliography}

1. Kantrowitz, A. 1972. Propulsion to orbit by ground-based lasers. Astronautics \& Aeronautics 10.5, pp. 74-75.

2. Larson, C W, Mead, Jr, F B and Kalliomaa, W M. 2003. Energy conversion in laser propulsion III. Beamed Energy Propulsion: First International Symposium on Beamed Energy Propulsion, CP664, pp. $170-181$.

3. Hecht, J. 1994. Understanding lasers: an entry-level guide. New York : Institute of Electrical and Electronics Engineers, Inc.

4. Phipps, C R, et al. 2002. Laser ablation powered mini-thruster. High Power Laser Ablation IV: Proceedings of the SPIE Vol. 4760. pp. 833-842.

5. Schall, W O, Eckel, H A and Bohn, W L. 2007. Laser propulsion thrusters for space transportation. [book auth.] C Phipps. Laser ablation and its applications. New York : Springer Science + Business Media LLC, pp. 435-454.

6. Sutton, G P and Biblarz, 0. 2001. Rocket propulsion elements. New York : John Wiley \& Sons,

7. Phipps, C R, et al. 2006. Performance test results for the laser-powered microthruster. Nara : American Institute of Physics, CP 830, Beamed Energy Propulsion: Fourth International Symposium. pp. 224-234.

8. Bayt, R L. 1999. Analysis, fabrication and testing of a MEMS-based micropropulsion system. Cambridge : MIT,

9. Goebel, D M and Katz, I. 2008. Fundamentals of electric propulsion: ion and hall thrusters. New Jersey : Wiley \& Sons inc.,

10. Zhang, K L, et al. 2005. A MEMS-based solid propellant microthruster with Au/Ti igniter. Sensors and Actuators A 122, pp. 113-123.

11. Chaalane, A, Rossi, C and Estève, D. 2007. The formulation and testing of new solid propellant mixture $(D B+x \% B P)$ for a new MEMS-based microthruster. Sensors and Actuators A 138, pp. 161-166. 
12. Birkan, M. 2008. Beamed energy propulsion: research status and needs- part 1. s.I. : American Institute of Physics, Beamed Energy Propulsion: Fifth International Symposium.

13. . Luke, J R, Phipps, C R and McDuff, G G. 2003. Laser plasma thrusterApplied Physics A, pp. 343-348.

14. Luke, J R and Phipps, C R. 2003. Laser plasma microthruster performance evaluation. [book auth.] A V Pakhomov. Beamed Energy Propulsion: First International Symposium on beamed energy propulsion.

s.I. : EBSCO publishing, pp. 223-229.

15. . Phipps, C R, et al. 2006. A ns-pulse laser microthrusterNara : American Institute of Physics, Beamed Energy Propulsion: Fourth International Symposium. pp. 235-246.

16. Horisawa, H and Kimura, I. 2002. Fundamental study on laser plasma accelerator for propulsion applications. Vacuum 65, pp. 389-396.

17. Pakhomov, A V, Gregory, D A and Thompson, M S. 2002. Specific impulse \& other characteristics of elementary propellants for ALP. AIAA, pp. 947-952.

18. Pakhomov, A V, et al. 2002. Ablative laser propulsion: specific impulse \& thrust derived from force measurements. AIAA, pp. 2305-2311.

19. Maesato, H, Koizumi, E and Tahara, H. 2006. Performance characteristics of low-power laser ablative thrusters for small satellites. Nara : American Institute of Physics. Beamed Energy Propulsion: Fourth International Symposium. pp. 284-294.

20. Yokoyama, S, et al. 2007. Fundamental study of laser micro propulsion using powdered-propellant. Florence : IEPC, The 30th International Electric Propulsion Conference. pp. 1-7.

21. Cai, J, Hu, $\mathbf{X}$ and Tang, Z. 2006. An experimental and conceptual investigation of laser micro propulsion. Nara : American Institute of Physics, Beamed Energy Propulsion: Fourth International Symposium. pp. 259-267.

22. Schwartz, M M. 1971. Laser welding and cutting. WRC Bulletin No. 167.

23. Yu, F TS and Khoo, I C. 1990. Principles of optical engineering. Canada : John Wiley \& Sons, Inc.

24. Wooten, F. 1972. Optical properties of solids. New York : Academic Press.

25. Eastham, D. 1986. Atomic physics of lasers. Philadelphia : Taylor \& Francis Inc. 
26. Milonni, P W and Eberly, J H. 1988. Lasers. Canada : John Wiley \& Sons, Inc.

27. Nair, K P R. 2006. Atoms, molecules and lasers. Oxford : Alpha Science International Ltd.,

28. Shimoda, K. 1983. Introduction to laser physics. Tokyo : Iwanami Shoten,

29. Gibbon, P. 2005. Short pulse laser interactions with matter. London : Imperial College Press,

30. Hirlimann, C. 2005. Laser basics. [book auth.] C Rullière. Femtosecond laser pulses. New York : Springer Science + Business Media LLC, pp. 1-23.

31. . Backus, S, et al. 1998. High power ultrafast lasers. Review of Scientific Instruments 69.3, pp. 12071223.

32. Chichkov, B N, et al. 1996. Femtosecond, picosecond and nanosecond laser ablation of solids. Applied Physics A 63, pp. 109-115.

33. Anisimov, S I and Khokhlov, V A. 1995. Instabilities in laser matter interaction. Florida : CRC Press, Inc.

34. Sudani, N. 2009. Thin wafer dicing using a high repetition rate femtosecond laser. Toronto : Ryerson University,

35. Mengqi, Y and Grigoropoulos, C P. 2001. Time-of-flight and emission spectroscopy study of femtosecond laser ablation of titanium. Journal of Applied Physics 89, pp. 5183-5190.

36. Panchatsharam, S. 2009. Fundamentals of mega hertz femtosecond laser ablation and its applications in interconnection via drilling. Toronto : Ryerson University,

37. Amaya, Phill. July 1, 2010. Structured fiber advances short-pulse laser performance. Laser Focus World. pp. 51-55.

38. Koechner, W. 2006. Solid state laser engineering, 6th ed. New York : Springer Science and Business Media, Inc.

39. Phipps, C R and Luke, J R. 2003. Advantages of a ns-pulse micro-laser plasma thruster. s.I. : American Institute of Physics, Beamed Energy Propulsion: First International Symposium on Beamed Energy Propulsion, CP664. pp. 230-239. 
40. Ziemer, J. K. 2002. Laser ablation microthruster technology. Maui : AIAA, 33rd AIAA Plasmadynamics and Lasers Conference. pp. 2002-2153.

41. . Siebold, M, et al. 2008. Diode-pumped lasers for ultra-high peak power. Applied Physics B, pp. 431437.

42. Treusch, G, Koenning, T and Shelef, B. 2008. High-power diode lasers boost power-beaming competition. Laser Focus World. March 1, pp. 75-79.

43. Coffey, V C. December 1, 2008. Femtosecond pulses reach terawatt power via Yb:CaF2. Laser Focus World. p. 37.

44. Kressel, H and Butler, J K. 1977. Semiconductor lasers and heterojunction LEDs. New York : Academic Press.

45. Ulm, T, et al. 2010. Femtosecond diode laser MOPA system at $920 \mathrm{~nm}$ based on asymmetric colliding pulse mode-locking. Applied Physics B, pp. 409-414.

46. Fiebig, C, et al. 2008. 12 W high-brightness single-frequency DBR tapered diode laser. Electronics Letters, pp. Vol. 44, No. 21.

47. Cai, J and Kanskar, M. 2009. 67\% CW power conversion efficiency from Al-free $1060 \mathrm{~nm}$ emitting diode lasers. Electronics Letters, pp. Vol. 45, No. 13.

48. Anisimov, S I, et al. 2007. Numerical simulation of the expansion into vacuum of a crystal heated by an ultrashort laser pulse. [book auth.] C Phipps. Laser ablation and its applications. New York : Springer Science + Business Media LLCpp. 1-16.

49. Amoruso, S, et al. 2002. Double-peak distribution of electron and ion emission profile during femtosecond laser ablation of metals. Applied Surface Science, pp. 358-363.

50. Schou, J, Amaruso, S and Lunney, J G. 2007. Plume dynamics. [book auth.] C Phipps. Laser ablation and its applications. New York : Springer Science + Business Media LLC, pp. 67-95.

51. Venkatakrishnan, K, et al. 2002. Femtosecond pulsed laser direct writing system. Optical Engineering 41, pp. 1441-1445. 
52. Afonso, C N, et al. 2007. Pulsed laser deposition for functional optical films. [book auth.] C Phipps. Laser Ablation and its Applications. New York : Springer Science + Business Media LLC., pp. 315-338.

53. Urech, L and Lippert, T. 2007. Designed polymers for ablation. [book auth.] C Phipps. Laser ablation and its applications. New York : Springer Science + Business Media LLC., pp. 281-297.

54. Zheng, Z Y, et al. 2006. Characteristic investigation of ablative laser propulsion driven by nanosecond laser pulses. Applied Physics A. pp. 329-332.

55. Eckel, H A, Tegel, J and Schall, W O. 2006. CO2 laser absorption in ablation plasmas. Nara : American Institute of Physics. Beamed Energy Propulsion: Fourth International Symposium. pp. 272-283.

56. Pakhomov, A V, Lin, J and Thompson, M S. 2004. Ablative laser propulsion: an update, part II. Sendai : American Institute of Physics. Beamed Energy Propulsion: Second International Symposium. pp. 178-189.

57. Sterling, E, et al. 2006. Laser driven mini-thrusters. Nara : American Institute of Physics. Beamed Energy Propulsion: Fourth International Symposium. pp. 247-258.

58. Zheng, Z Y, et al. 2006. Enhancement of coupling coefficient of laser plasma propulsion by water confinement. Applied Physics A 85, pp. 441-443.

59. Kautek, W. 2007. Physical chemistry of ultrafast laser interactions with solids. [book auth.] C Phipps. Laser ablation and its applications. New York : Springer Science + Business Media LLC., pp. 215-229.

60. Urech, L, et al. 2007. Polymer ablation: from fundamentals of polymer design to laser plasma thruster. Applied Surface Science 253, pp. 6409-6415.

61. White, F M. 2003. Fluid Mechanics fifth edition. New York : Mcgraw-Hill,

62. Uchida, S and Yabe, T. 2007. Laser propulsion. [book auth.] C Phipps. Laser ablation and its applications. New York : Springer Science + Business Media LLC., pp. 455-471.

63. Phipps, C R and Michaelis, M M. 1994. LISP: laser impulse space propulsion. Laser and Particle Beams Vol. 12, pp. 23-54.

64. Measurement Specialties, Inc. 2006. Piezo film sensors technical manual. Hampton : s.n. 
65. Mandelis, A and Christofides, C. 1993. Physics, chemistry, and technology of solid state gas sensor devices. New York : John Wiley \& Sons, Inc.

66. Naranjo-Bueno, M F. 2007. Design of PVDF transducers for acoustic reflectometry applications. Mayaguez : University of Puerto Rico.

67. Cady, W G. 1964. Piezoelectricity: an introduction to the theory \& applications of electromechanical phenomena in crystals. Toronto : General Publishing Company Ltd.

68. Katzir, S. 2006. The beginnings of piezoelectricity: a study in mundane physics. Netherlands : Springer 69. Vargas, E, et al. 2008. High-voltage, high-frequency amplifier drives piezoelectric PVDF transducer. Electronics Design, Strategy, News. July 24, p. 57.

70. Sivakumar, M, Tan, B and Venkatakrishnan, K. 2010. Enhancement of silicon nanostructures generation using dual wavelength double pulse femtosecond laser under ambient condition. Journal of Applied Physics 107, pp. 044307 (1-3).

71. Thermal conductivity of some common materials. The Engineering Toolbox. [Online] [Cited: July 1, 2010.] http://www.engineeringtoolbox.com/thermal-conductivity-d_429.html. 